Supporting Information

\title{
Labdanes, Withanolides and Other Constituents from Physalis nicandroides
}

Fernando R. Torres,${ }^{\dagger}$ Ana L. Pérez-Castorena,${ }^{\dagger}$ Laura Arredondo,${ }^{\dagger}$ Rubén A. Toscano, ${ }^{\dagger}$ Antonio Nieto-Camacho, ${ }^{\dagger}$ Mahinda Martínez, ${ }^{\ddagger}$ and Emma Maldonado*广

†Instituto de Química, Universidad Nacional Autónoma de México, Circuito Exterior, Ciudad Universitaria, Coyoacán, 04510, Cd. Mx., México.

*Facultad de Ciencias Naturales, Universidad Autónoma de Querétaro, Avenida de las Ciencias s/n, Col. Juriquilla 76230 , Querétaro, Qro, México. 
Table S1. ${ }^{1} \mathrm{H}$ NMR data of Compounds $1 \mathbf{a}, 2 \mathbf{a}$ and $6 \mathbf{a} / 7 \mathbf{a}$.

Table S2. ${ }^{13} \mathrm{C}$ NMR data of Compounds 1a, 2a and 6a/7a.

Figure S1. ${ }^{1} \mathrm{H}$ NMR spectrum of compound $1\left(500 \mathrm{MHz}, \mathrm{CDCl}_{3}\right)$

Figure S2. ${ }^{13} \mathrm{C}$ NMR spectrum of compound $\mathbf{1}\left(125 \mathrm{MHz}, \mathrm{CDCl}_{3}\right)$

Figure S3. COSY spectrum of compound $1\left(\mathrm{CDCl}_{3}\right)$

Figure S4. HSQC spectrum of compound $\mathbf{1}\left(\mathrm{CDCl}_{3}\right)$

Figure S5. $\mathrm{HMBC}$ spectrum of compound $1\left(\mathrm{CDCl}_{3}\right)$

Figure S6. NOESY spectrum of compound $\mathbf{1}\left(\mathrm{CDCl}_{3}\right)$

Figure S7. ${ }^{1} \mathrm{H}$ NMR spectrum of compound $2\left(500 \mathrm{MHz}, \mathrm{CDCl}_{3}\right)$

Figure S8. ${ }^{13} \mathrm{C}$ NMR spectrum of compound $2\left(125 \mathrm{MHz}, \mathrm{CDCl}_{3}\right)$

Figure S9. COSY spectrum of compound $2\left(\mathrm{CDCl}_{3}\right)$

Figure S10. HSQC spectrum of compound $2\left(\mathrm{CDCl}_{3}\right)$

Figure S11. HMBC spectrum of compound $2\left(\mathrm{CDCl}_{3}\right)$

Figure S12. NOESY spectrum of compound $2\left(\mathrm{CDCl}_{3}\right)$

Figure S13. ${ }^{1} \mathrm{H}$ NMR spectrum of compound $3\left(500 \mathrm{MHz}, \mathrm{CDCl}_{3}\right)$

Figure S14. ${ }^{13} \mathrm{C}$ NMR spectrum of compound $3\left(125 \mathrm{MHz}, \mathrm{CDCl}_{3}\right)$

Figure S15. COSY spectrum of compound $3\left(\mathrm{CDCl}_{3}\right)$

Figure S16. $\mathrm{HSQC}$ spectrum of compound $\mathbf{3}\left(\mathrm{CDCl}_{3}\right)$

Figure S17. $\mathrm{HMBC}$ spectrum of compound $\mathbf{3}\left(\mathrm{CDCl}_{3}\right)$

Figure S18. ${ }^{1} \mathrm{H}$ NMR spectrum of compound $\mathbf{1 a}\left(500 \mathrm{MHz}, \mathrm{CDCl}_{3}\right)$ 
Figure S20. ${ }^{1} \mathrm{H}$ NMR spectrum of compound $2 \mathbf{a}\left(500 \mathrm{MHz}, \mathrm{CDCl}_{3}\right)$

Figure S21. ${ }^{13} \mathrm{C}$ NMR spectrum of compound $2 \mathbf{a}\left(125 \mathrm{MHz}, \mathrm{CDCl}_{3}\right)$

Figure S22. ${ }^{1} \mathrm{H}$ NMR spectrum of compound $\mathbf{3 a}\left(500 \mathrm{MHz}, \mathrm{CDCl}_{3}\right)$

Figure S23. ${ }^{1} \mathrm{H}$ NMR spectrum of compound $4\left(500 \mathrm{MHz}, \mathrm{CDCl}_{3}\right)$

Figure S24. ${ }^{13} \mathrm{C}$ NMR spectrum of compound $\mathbf{4}\left(125 \mathrm{MHz}, \mathrm{CDCl}_{3}\right)$

Figure S25. COSY spectrum of compound $4\left(\mathrm{CDCl}_{3}\right)$

Figure S26. HSQC spectrum of compound $4\left(\mathrm{CDCl}_{3}\right)$

Figure S27. $\mathrm{HMBC}$ spectrum of compound $4\left(\mathrm{CDCl}_{3}\right)$

Figure S28. NOESY spectrum of compound $4\left(\mathrm{CDCl}_{3}\right)$

Figure S29. ${ }^{1} \mathrm{H}$ NMR spectrum of compound $4 \mathrm{a}\left(500 \mathrm{MHz}, \mathrm{CDCl}_{3}\right)$

Figure S30. ${ }^{13} \mathrm{C}$ NMR spectrum of compound $4 \mathrm{a}\left(125 \mathrm{MHz}, \mathrm{CDCl}_{3}\right)$

Figure S31. ${ }^{1} \mathrm{H}$ NMR spectrum of compound $5\left(500 \mathrm{MHz}, \mathrm{CDCl}_{3}\right)$

Figure S32. ${ }^{13} \mathrm{C}$ NMR spectrum of compound $\mathbf{5}\left(125 \mathrm{MHz}, \mathrm{CDCl}_{3}\right)$

Figure S33. COSY spectrum of compound $\mathbf{5}\left(\mathrm{CDCl}_{3}\right)$

Figure S34. HSQC spectrum of compound $\mathbf{5}\left(\mathrm{CDCl}_{3}\right)$

Figure S35. ${ }^{1} \mathrm{H}$ NMR spectrum of compound $\mathbf{5 a}\left(500 \mathrm{MHz}, \mathrm{CDCl}_{3}\right)$

Figure S36. ${ }^{13} \mathrm{C}$ NMR spectrum of compound $\mathbf{5 a}\left(125 \mathrm{MHz}, \mathrm{CDCl}_{3}\right)$ 
Figure S38. $\mathrm{HSQC}$ spectrum of compound $\mathbf{5 a}\left(\mathrm{CDCl}_{3}\right)$

Figure S40. NOESY spectrum of compound $\mathbf{5 a}\left(\mathrm{CDCl}_{3}\right)$

Figure S41. ${ }^{1} \mathrm{H}$ NMR spectra of natural (A) and semisynthetic (B) compounds $6 / 7\left(500 \mathrm{MHz}, \mathrm{CDCl}_{3}\right)$

Figure S42. ${ }^{13} \mathrm{C}$ NMR spectra of natural (A) and semisynthetic (B) compounds $6 / 7\left(125 \mathrm{MHz}, \mathrm{CDCl}_{3}\right)$

Figure S43. COSY spectrum of compounds $6 / 7\left(\mathrm{CDCl}_{3}\right)$

Figure S44. HSQC spectrum of compounds $6 / 7\left(\mathrm{CDCl}_{3}\right)$

Figure S45. HMBC spectrum of compounds $6 / 7\left(\mathrm{CDCl}_{3}\right)$

Figure S46. ${ }^{1} \mathrm{H}$ NMR spectrum of compounds $6 \mathbf{a} / 7 \mathbf{a}\left(500 \mathrm{MHz}, \mathrm{CDCl}_{3}\right)$

Figure S47. ${ }^{13} \mathrm{C}$ NMR spectrum of compounds $6 \mathbf{a} / 7 \mathbf{a}\left(125 \mathrm{MHz}, \mathrm{CDCl}_{3}\right)$

Figure S48. ${ }^{1} \mathrm{H}$ NMR spectra of compound 8 from natural (A) and semisynthetic 6/7 (B) [500 (A), 300 (B) MHz, CDCl $]$.

Figure S49. ${ }^{13} \mathrm{C}$ NMR spectrum of compound $8\left(125 \mathrm{MHz}, \mathrm{CDCl}_{3}\right)$

Figure S50. ${ }^{1} \mathrm{H}$ NMR spectrum of compound $9\left(500 \mathrm{MHz}, \mathrm{CDCl}_{3}\right)$

Figure S51. ${ }^{13} \mathrm{C}$ NMR spectrum of compound $\mathbf{9}\left(125 \mathrm{MHz}, \mathrm{CDCl}_{3}\right)$

Figure S52. COSY spectrum of compound $\mathbf{9}\left(\mathrm{CDCl}_{3}\right)$

Figure S53. HSQC spectrum of compound $9\left(\mathrm{CDCl}_{3}\right)$

Figure S54. HMBC spectrum of compound $9\left(\mathrm{CDCl}_{3}\right)$

Figure S55. NOESY spectrum of compound $9\left(\mathrm{CDCl}_{3}\right)$

Figure S56. ${ }^{1} \mathrm{H}$ NMR spectrum of compound $14\left(500 \mathrm{MHz}, \mathrm{CDCl}_{3}\right)$ 
Figure S58. COSY spectrum of compound $14\left(\mathrm{CDCl}_{3}\right)$

Figure S59. HSQC spectrum of compound $14\left(\mathrm{CDCl}_{3}\right)$

Figure S60. HMBC spectrum of compound $14\left(\mathrm{CDCl}_{3}\right)$

Figure S61. NOESY spectrum of compound $14\left(\mathrm{CDCl}_{3}\right)$

Figure S62. ${ }^{1} \mathrm{H}$ NMR spectrum of compound $15\left(500 \mathrm{MHz}, \mathrm{CDCl}_{3}\right)$

Figure S63. ${ }^{13} \mathrm{C}$ NMR spectrum of compound $15\left(125 \mathrm{MHz}, \mathrm{CDCl}_{3}\right)$

Figure S64. COSY spectrum of compound $15\left(\mathrm{CDCl}_{3}\right)$

Figure S65. HSQC spectrum of compound $15\left(\mathrm{CDCl}_{3}\right)$

Figure S66. HMBC spectrum of compound $15\left(\mathrm{CDCl}_{3}\right)$

Figure S67. NOESY spectrum of compound $15\left(\mathrm{CDCl}_{3}\right)$

Figure S68. ${ }^{1} \mathrm{H}$ NMR spectrum of compound $16\left(500 \mathrm{MHz}, \mathrm{CDCl}_{3}\right)$

Figure S69. ${ }^{13} \mathrm{C}$ NMR spectrum of compound $16\left(125 \mathrm{MHz}, \mathrm{CDCl}_{3}\right)$

Figure S70. COSY spectrum of compound $16\left(\mathrm{CDCl}_{3}\right)$

Figure S71. HSQC spectrum of compound $16\left(\mathrm{CDCl}_{3}\right)$

Figure S72. HMBC spectrum of compound $16\left(\mathrm{CDCl}_{3}\right)$

Figure S73. NOESY spectrum of compound $16\left(\mathrm{CDCl}_{3}\right)$

Figure S74. ${ }^{1} \mathrm{H}$ NMR spectrum of compound $\mathbf{1 6 a}\left(500 \mathrm{MHz}, \mathrm{CDCl}_{3}\right)$

Figure S75. ${ }^{13} \mathrm{C}$ NMR spectrum of compound $\mathbf{1 6 a}\left(125 \mathrm{MHz}, \mathrm{CDCl}_{3}\right)$

Figure S76 and Table S3. Energy-minimized 3D structure of compound 15 showing selected H-H distances. 
Table S1. ${ }^{1} \mathrm{H}$ NMR Data of Compounds $1 \mathrm{a}, 2 \mathrm{a}$, and 6a/7a. $\left(500 \mathrm{MHz}, \mathrm{CDCl}_{3}\right)$.

\begin{tabular}{|c|c|c|c|}
\hline $\begin{array}{l}\text { posit } \\
\text { ion }\end{array}$ & $\mathbf{1 a}$ & $2 \mathbf{a}$ & $\mathbf{6 a} / 7 \mathbf{a}$ \\
\hline \multirow[t]{2}{*}{1} & 1.01 ddd $(13.0,13.0,4.0)$ & 0.91 ddd $(13.0,13.0,4.0)$ & $1.01 / 1.03 \mathrm{~m}$ \\
\hline & $1.73 \mathrm{~m}$ & $1.70 \mathrm{~m}$ & $1.75 \mathrm{~m}$ \\
\hline \multirow[t]{2}{*}{2} & $1.52 \mathrm{~m}$ & $1.48 \mathrm{~m}$ & $1.52 \mathrm{~m}$ \\
\hline & $1.52 \mathrm{~m}$ & $1.56 \mathrm{~m}$ & $1.57 \mathrm{~m}$ \\
\hline \multirow[t]{2}{*}{3} & 1.19 ddd $(13.5,13.5,4.0)$ & 1.16 ddd $(13.0,13.0,4.0)$ & $1.19 \mathrm{~m}$ \\
\hline & $\begin{array}{l}1.40 \text { dddd }(13.0,3.0,3.0, \\
1.5)\end{array}$ & 1.39 dddd $(13.0,3.5,3.5,1.5)$ & $1.41 \mathrm{~m}$ \\
\hline 5 & $1.09 \mathrm{dd}(12.5,2.5)$ & $1.04 \mathrm{dd}(13.0,3.0)$ & $1.10 / 1.09 \mathrm{dd}(12.5,2.5)$ \\
\hline \multirow[t]{2}{*}{6} & $1.73 \mathrm{~m}$ & $1.71 \mathrm{~m}$ & $1.73 \mathrm{~m}$ \\
\hline & $\begin{array}{l}1.31 \text { dddd (13.0,13.0,13.0, } \\
4.0)\end{array}$ & 1.29 dddd $(13.0,13.0,13.0,4.0)$ & $1.94 \mathrm{~m}$ \\
\hline \multirow[t]{2}{*}{7} & 1.95 ddd $(13.0,13.0,5.0)$ & 1.86 br ddd $13.0,13.0,4.5$ & $2.05 \mathrm{~m}$ \\
\hline & 2.39 ddd $(13.0,4.0,2.5)$ & 2.36 ddd $13.0,4.0,2.5$ & 2.39 br d (13.0) \\
\hline 9 & 1.66 br d 11.0 & 1.43 br t 6.7 & $1.70 \mathrm{~m}$ \\
\hline \multicolumn{4}{|l|}{10} \\
\hline \multirow[t]{2}{*}{11} & 1.79 ddd $(14.0,10.0,1.5)$ & $1.73 \mathrm{~m}$ & $1.80 / 1.94 \mathrm{~m}$ \\
\hline & 1.58 ddd $(14.0,11.0,2.5)$ & $1.73 \mathrm{~m}$ & $1.80 / 1.68 \mathrm{~m}$ \\
\hline 12 & 5.14 br dd $(10.0,1.5)$ & $5.27 \mathrm{dd}(8.5,7.0)$ & $5.10 \mathrm{dd} / 5.34 \mathrm{~m}(10.0,2.5)$ \\
\hline 14 & $5.55 \operatorname{tdq}(7.0,1.5,0.5)$ & $5.49 \operatorname{tdq}(6.5,1.5,0.5)$ & $\begin{array}{l}5.38 / 5.56 \text { br dd }(8.0,2.5 / 8.5 \text {, } \\
2.0)\end{array}$ \\
\hline \multirow{2}{*}{15} & 4.60d (7.0) & 4.64 ddd $(13.0,7.0,1.0)$ & 4.39/4.29 dd (12.0,3.0) \\
\hline & 4.60d (7.0) & 4.57 ddd $(13.0,7.0,1.0)$ & 4.10/4.14 dd $(12.0,3.0)$ \\
\hline 16 & $1.70 \mathrm{dt}(1.5,0.5)$ & $1.68 \mathrm{dt}(1.5,1.0)$ & $\begin{array}{l}5.25 / 5.33 \mathrm{~s} \\
5.22 / 5.29 \mathrm{~s}\end{array}$ \\
\hline 17 & $\begin{array}{l}4.86 \mathrm{~m} \\
4.47 \mathrm{br} \mathrm{d}(1.0)\end{array}$ & $\begin{array}{l}4.88 \text { br d (1.5) } \\
4.79 \text { br d (1.0) }\end{array}$ & $\begin{array}{l}4.89 / 4.87 \text { br s } \\
4.58 / 4.51 \text { br s }\end{array}$ \\
\hline 18 & $0.88 \mathrm{~s}$ & $0.86 \mathrm{~s}$ & $0.88 \mathrm{~s}$ \\
\hline 19 & $0.80 \mathrm{~s}$ & $0.79 \mathrm{~s}$ & $0.80 \mathrm{~s}$ \\
\hline 20 & $0.66 \mathrm{~s}$ & $0.68 \mathrm{~s}$ & $0.68 / 0.67 \mathrm{~s}$ \\
\hline Ac & $2.058 \mathrm{~s}, 2.055 \mathrm{~s}$ & $2.05 \mathrm{~s}, 2.02 \mathrm{~s}$ & $\begin{array}{l}2.10,2.09,2.07,2.064,2.058, \\
2.05\end{array}$ \\
\hline
\end{tabular}


Table S2. ${ }^{13} \mathrm{C}$ NMR Data of Compounds 1a, 2a, and $6 \mathrm{a} / 7 \mathrm{a} .\left(\mathrm{CDCl}_{3}, 125 \mathrm{MHz}\right)$.

\begin{tabular}{|c|c|c|c|}
\hline $\begin{array}{l}\text { positio } \\
\text { n }\end{array}$ & $\mathbf{1 a}$ & $2 \mathbf{a}$ & $6 \mathbf{a} / 7 \mathbf{a}$ \\
\hline $1 \mathrm{CH}_{2}$ & 39.1 & 39.1 & $39.0 / 39.1$ \\
\hline $2 \mathrm{CH}_{2}$ & 19.3 & 19.4 & 19.3 \\
\hline $3 \mathrm{CH}_{2}$ & 42.1 & 42.1 & $42.09 / 42.12$ \\
\hline $4 \mathrm{C}$ & 33.6 & 33.5 & 33.6 \\
\hline $5 \mathrm{CH}$ & 55.5 & 55.6 & 55.6 \\
\hline $6 \mathrm{CH}_{2}$ & 24.3 & 24.3 & $24.4 / 24.3$ \\
\hline $7 \mathrm{CH}_{2}$ & 38.2 & 38.2 & $38.2 / 38.1$ \\
\hline $8 \mathrm{C}$ & 148.3 & 147.5 & $148.0 / 148.2$ \\
\hline $9 \mathrm{CH}$ & 52.6 & 52.5 & $52.8 / 52.7$ \\
\hline $10 \mathrm{C}$ & 39.3 & 39.3 & $39.33 / 39.30$ \\
\hline $11 \mathrm{CH}_{2}$ & 28.2 & 26.2 & $29.3 / 29.1$ \\
\hline $12 \mathrm{CH}$ & 76.9 & 78.7 & $73.2 / 73.3$ \\
\hline $13 \mathrm{C}$ & 140.2 & 138.2 & $146.2 / 144.5$ \\
\hline $14 \mathrm{CH}$ & 120.1 & 123.5 & 71.1 \\
\hline $15 \mathrm{CH}_{2}$ & 60.8 & 60.6 & $65.03 / 64.95$ \\
\hline $16 \mathrm{CH}_{3}$ & 12.8 & 11.4 & $113.5 / 116.4^{a}$ \\
\hline $17 \mathrm{CH}_{2}$ & 106.6 & 107.4 & $106.8 / 106.7$ \\
\hline $18 \mathrm{CH}_{3}$ & 33.6 & 33.5 & 33.5 \\
\hline $19 \mathrm{CH}_{3}$ & 21.7 & 21.7 & 21.7 \\
\hline $20 \mathrm{CH}_{3}$ & 14.5 & 14.6 & $14.53 / 14.48$ \\
\hline & 171.0, & 170.8 & 170.6 (x2), 170.4, \\
\hline & 170.4, 21.3, & 170.1, 21.0, & $170.3,169.9,169.8$ \\
\hline & 21.0 & 19.0 & \\
\hline & & & $21.2,21.17,21.1,2$ \\
\hline & & & $1.0,20.8,20.7$ \\
\hline
\end{tabular}

${ }^{\mathrm{a}} \mathrm{CH}_{2}$. 

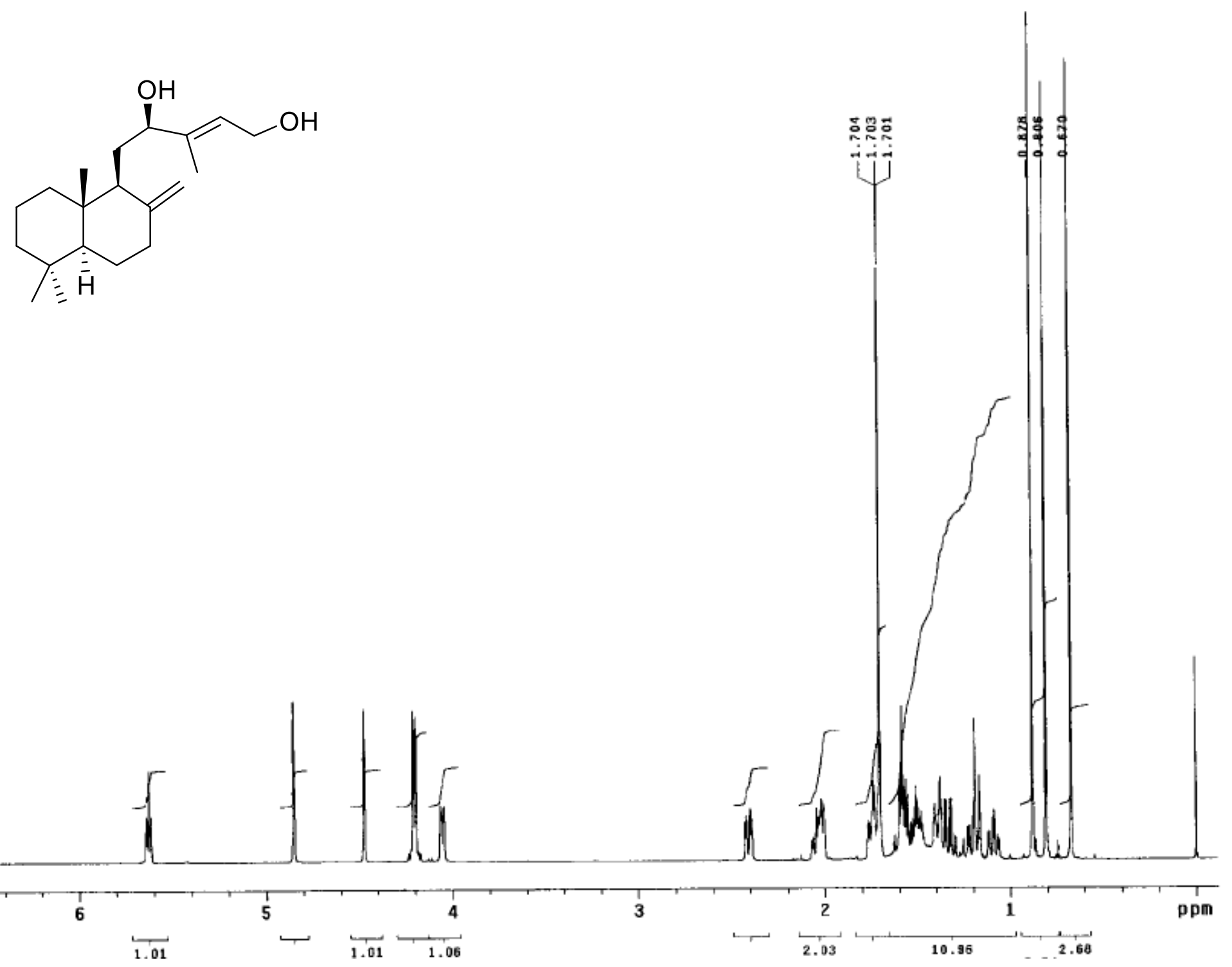

Figure S1. ${ }^{1} \mathrm{H}$ NMR spectrum of compound $1\left(500 \mathrm{MHz}, \mathrm{CDCl}_{3}\right)$. 


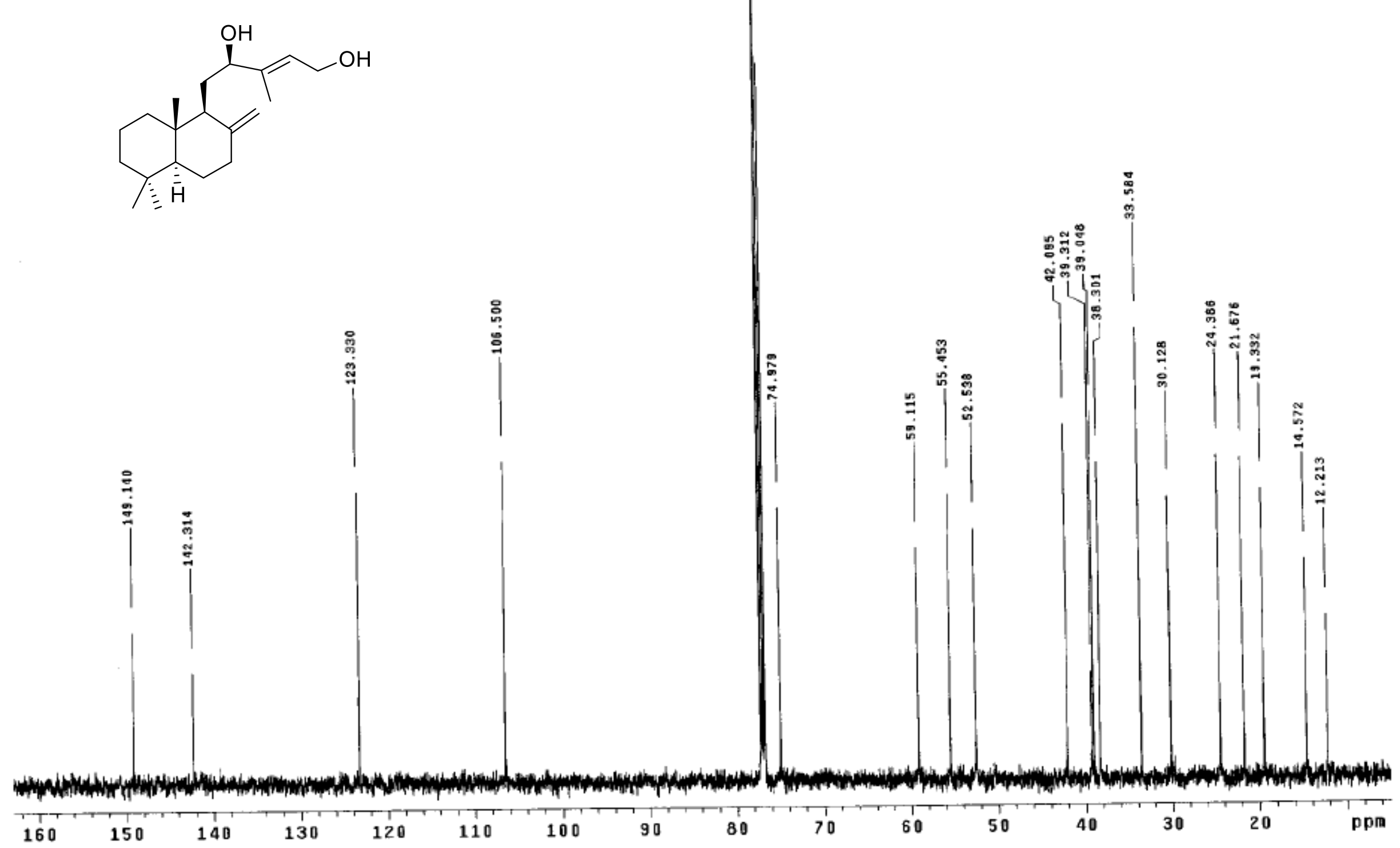

Figure S2. ${ }^{13} \mathrm{C}$ NMR spectrum of compound $\mathbf{1}\left(125 \mathrm{MHz}, \mathrm{CDCl}_{3}\right)$. 

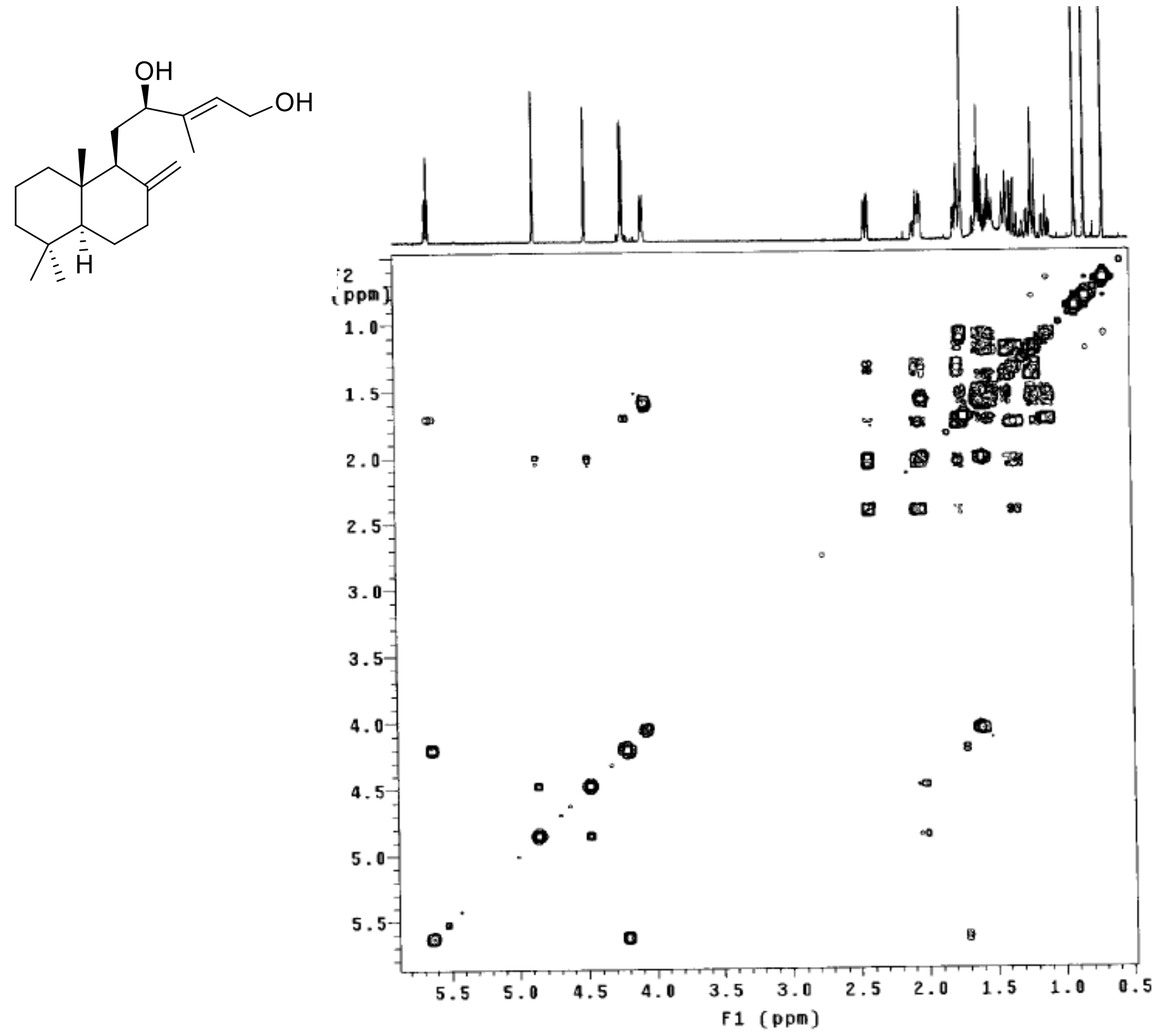

Figure S3. COSY spectrum of compound $\mathbf{1}\left(\mathrm{CDCl}_{3}\right)$. 

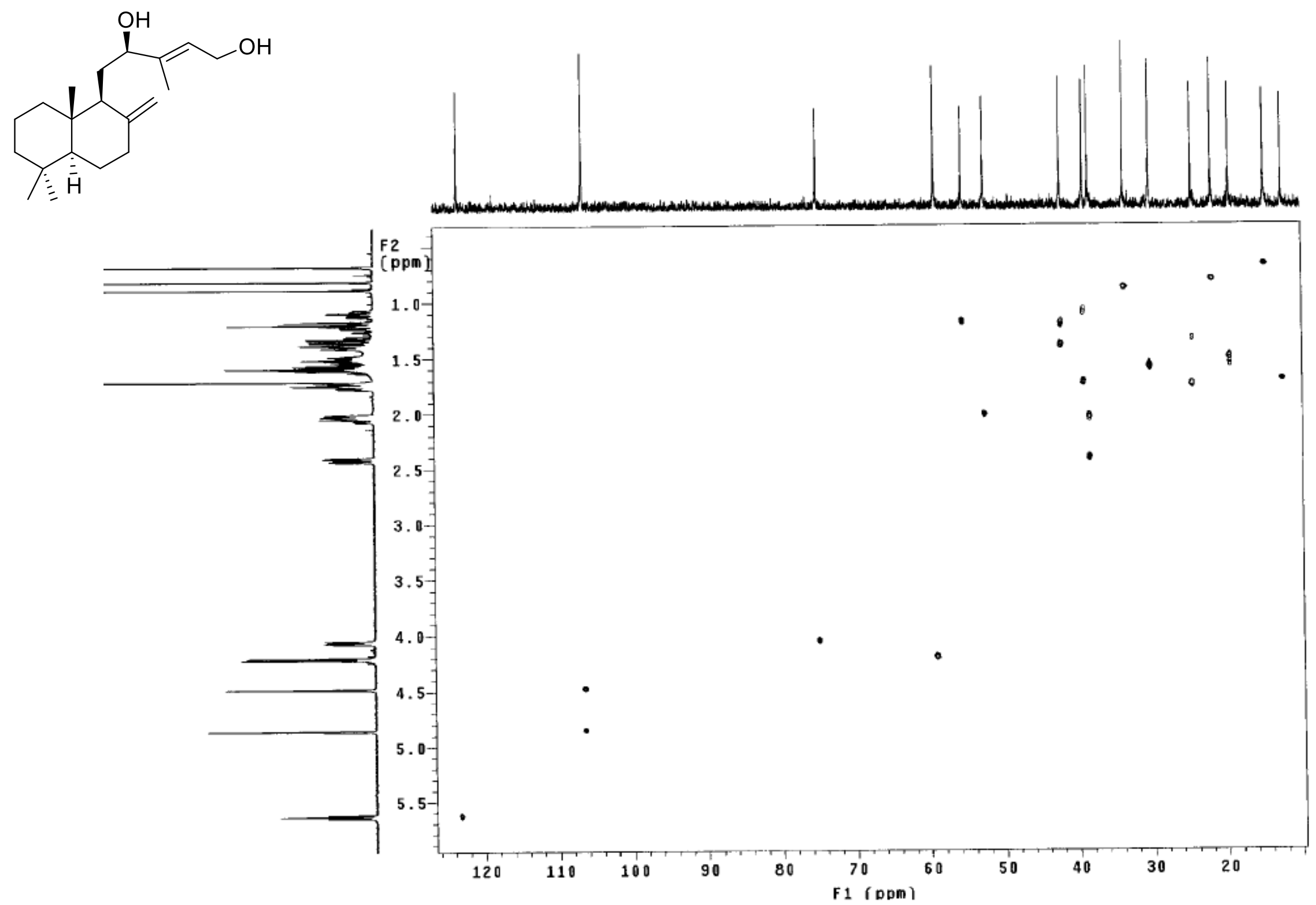

Figure S4. HSQC spectrum of compound $\mathbf{1}\left(\mathrm{CDCl}_{3}\right)$. 


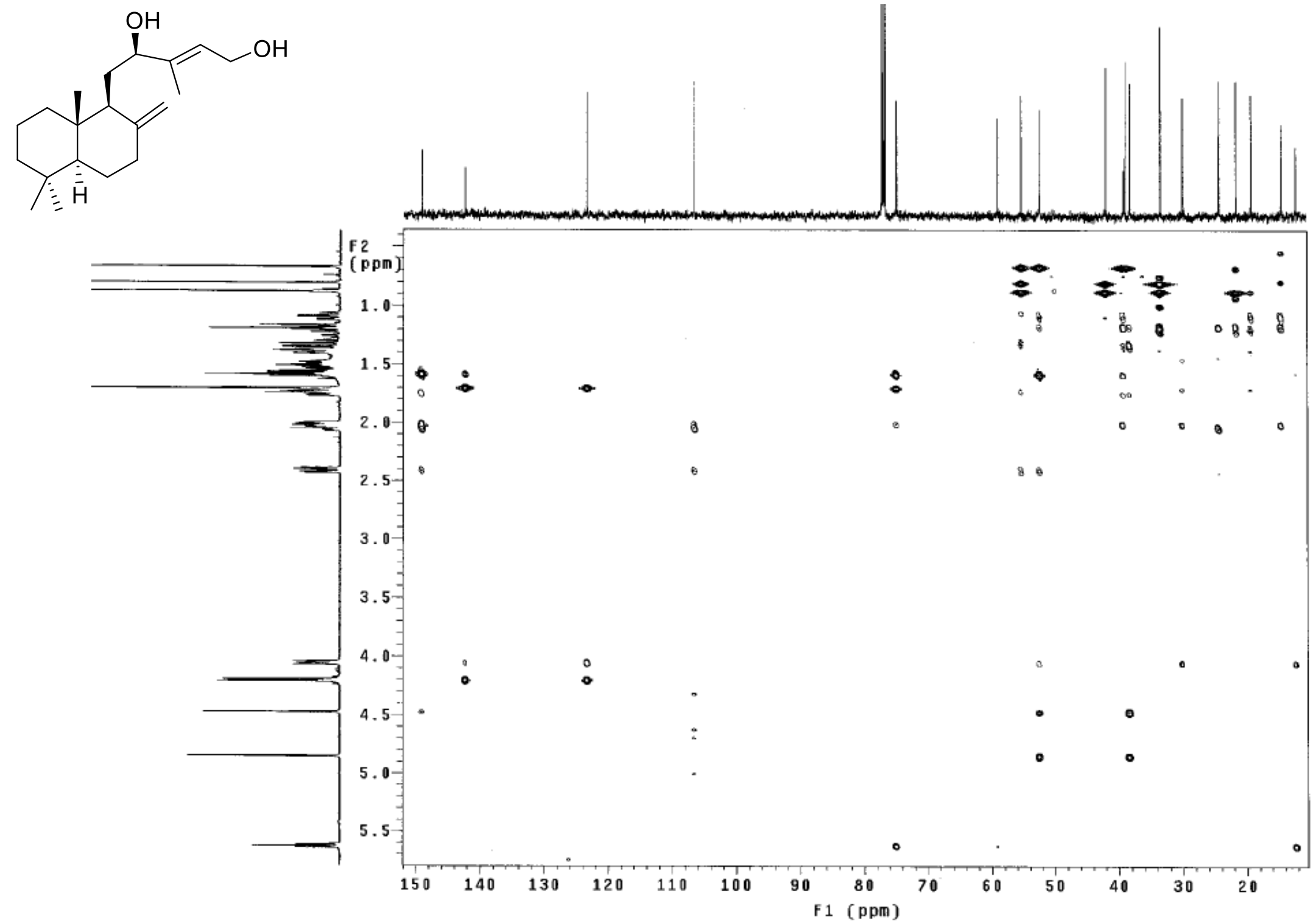

Figure S5. HMBC spectrum of compound $\mathbf{1}\left(\mathrm{CDCl}_{3}\right)$. 

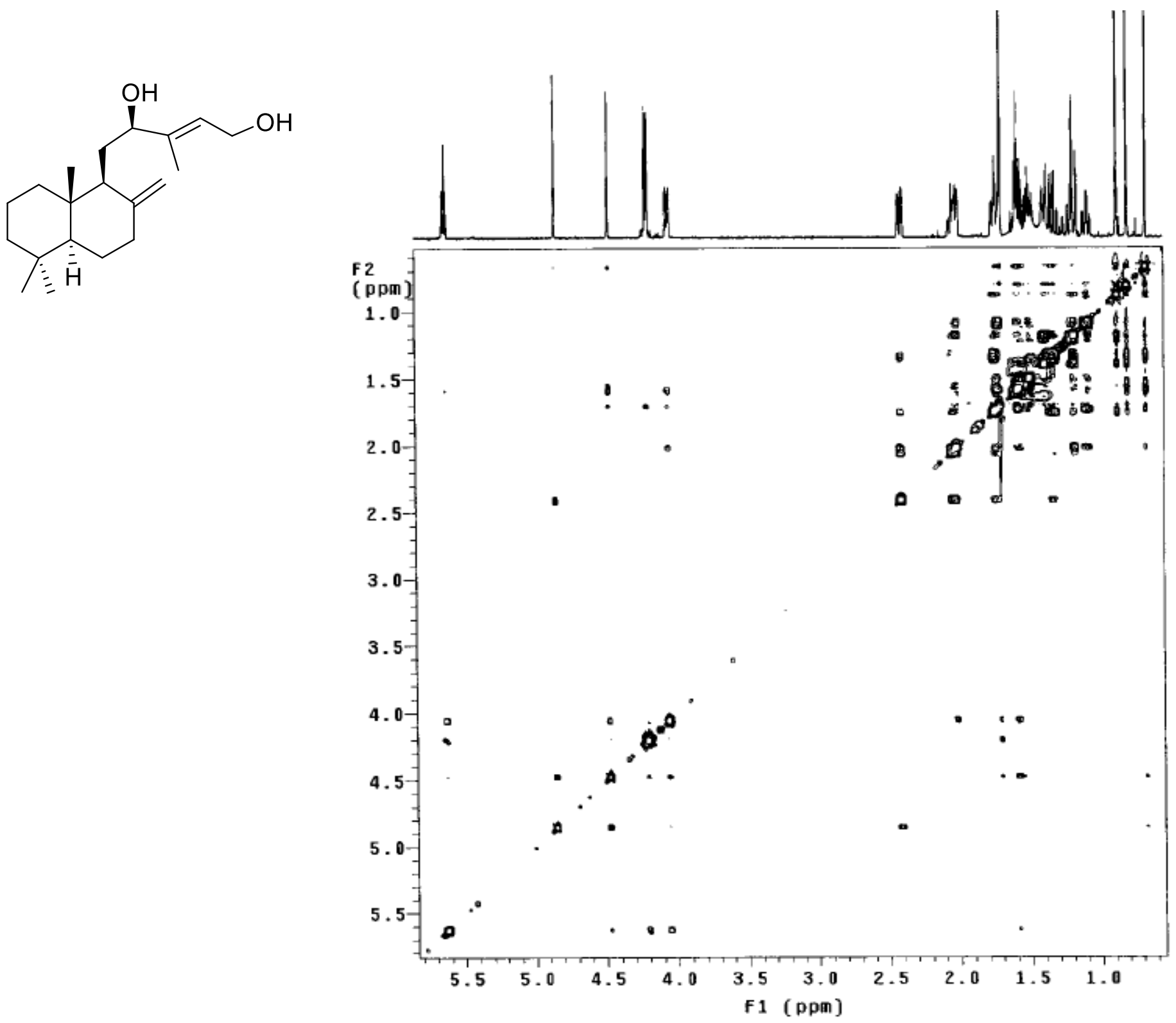

Figure S6. NOESY spectrum of compound $\mathbf{1}\left(\mathrm{CDCl}_{3}\right)$. 

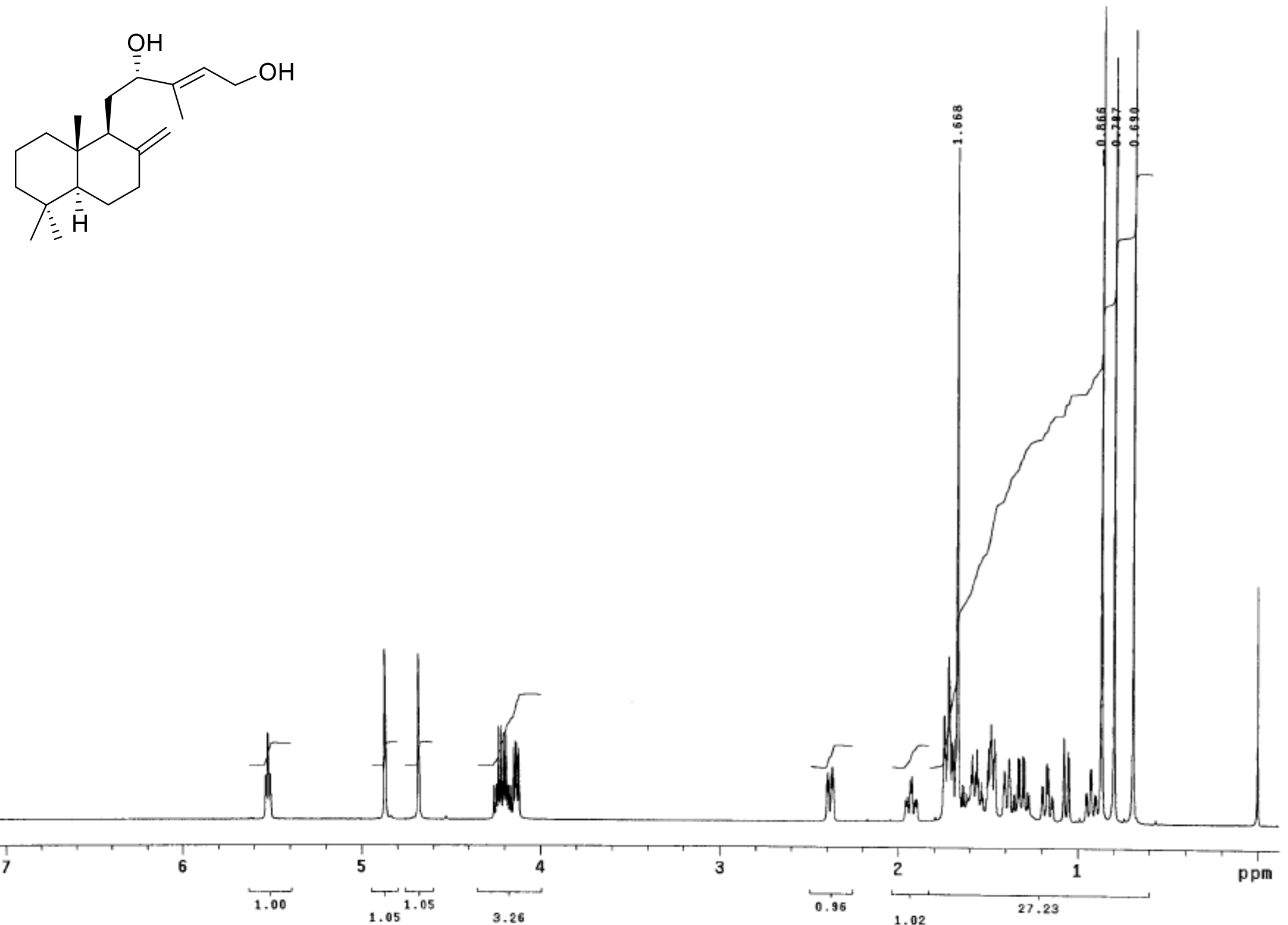

Figure S7. ${ }^{1} \mathrm{H}$ NMR spectrum of compound $2\left(500 \mathrm{MHz}, \mathrm{CDCl}_{3}\right)$. 


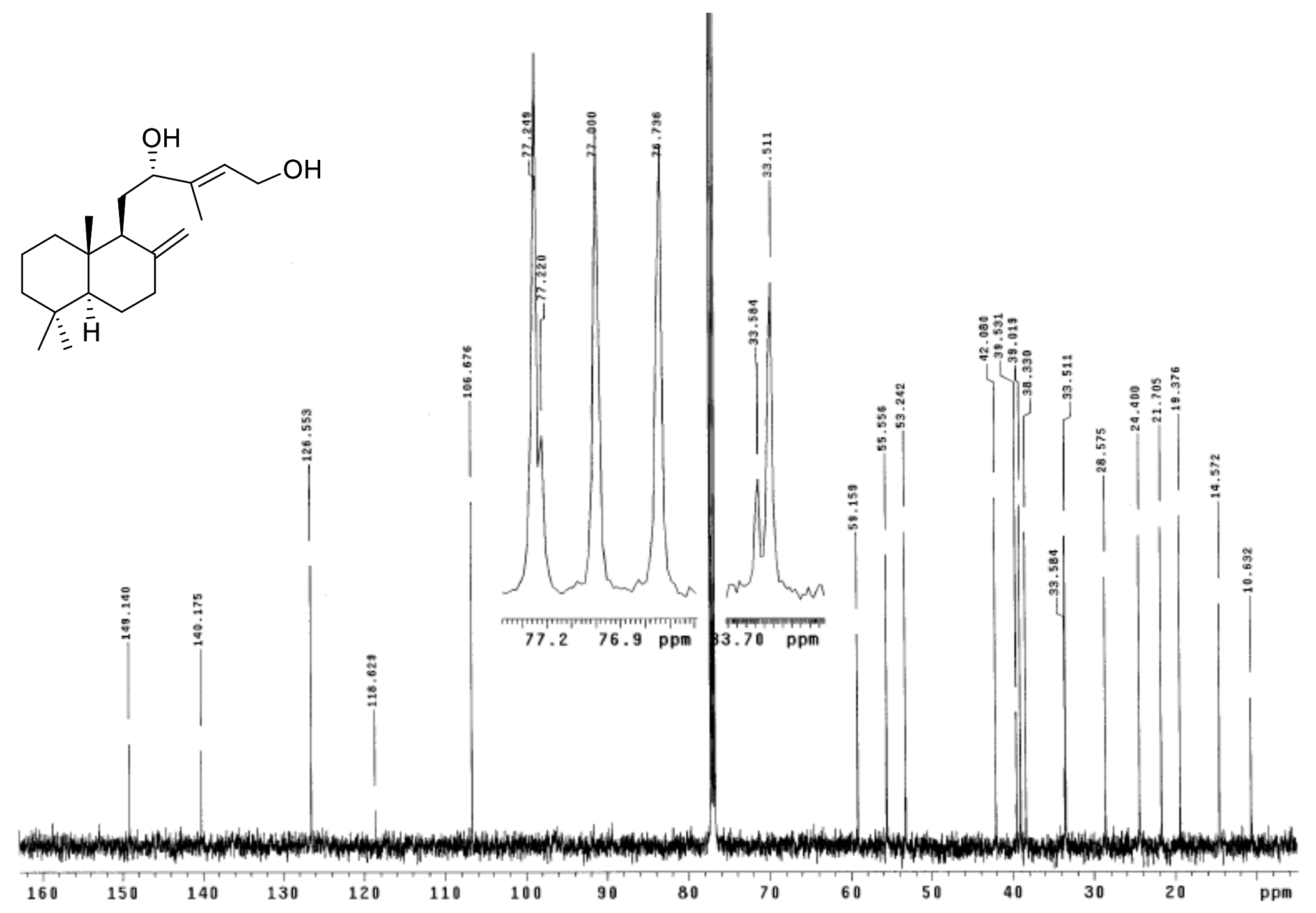

Figure S8. ${ }^{13} \mathrm{C}$ NMR spectrum of compound $2\left(125 \mathrm{MHz}, \mathrm{CDCl}_{3}\right)$. 

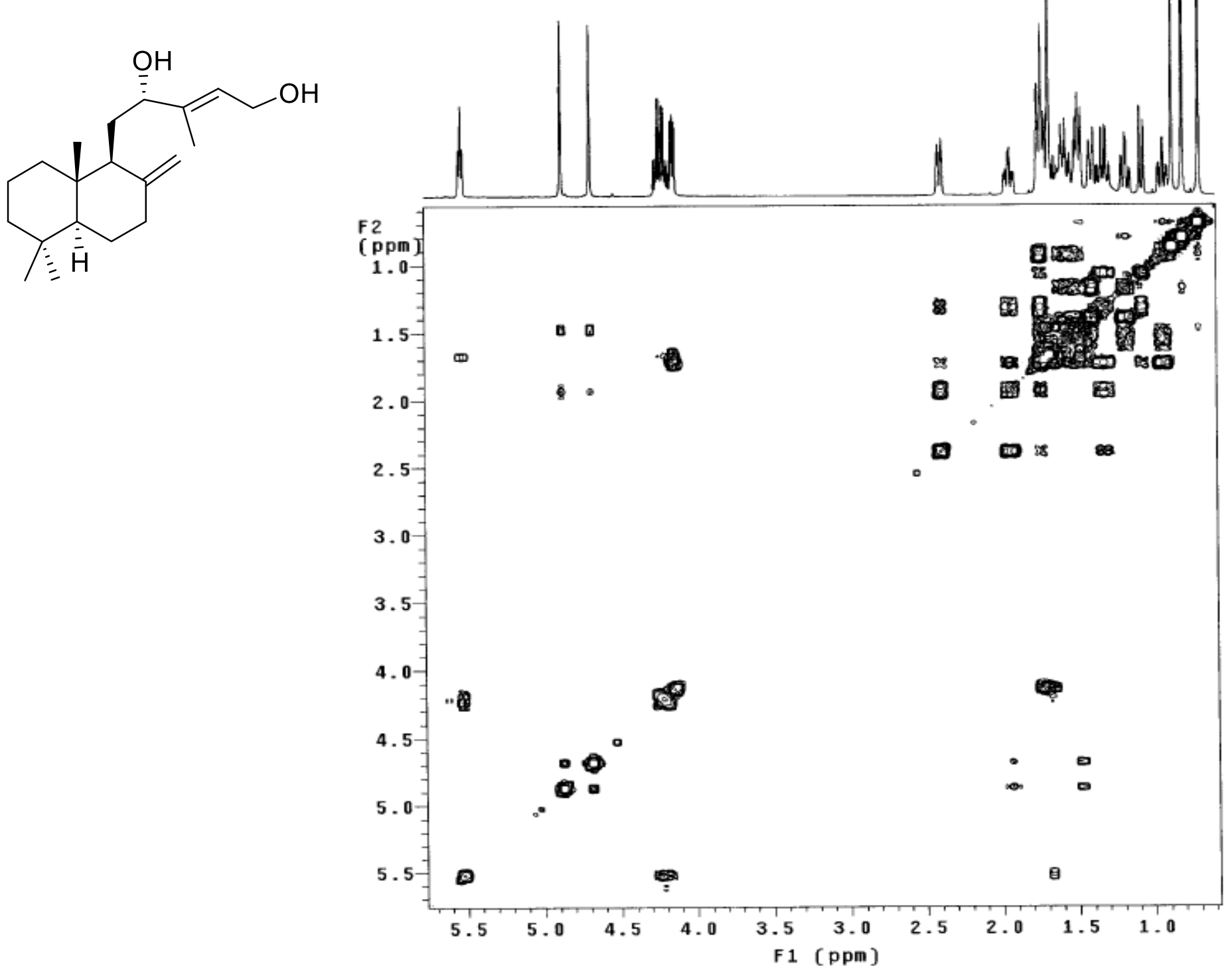

Figure S9. COSY spectrum of compound $2\left(\mathrm{CDCl}_{3}\right)$. 

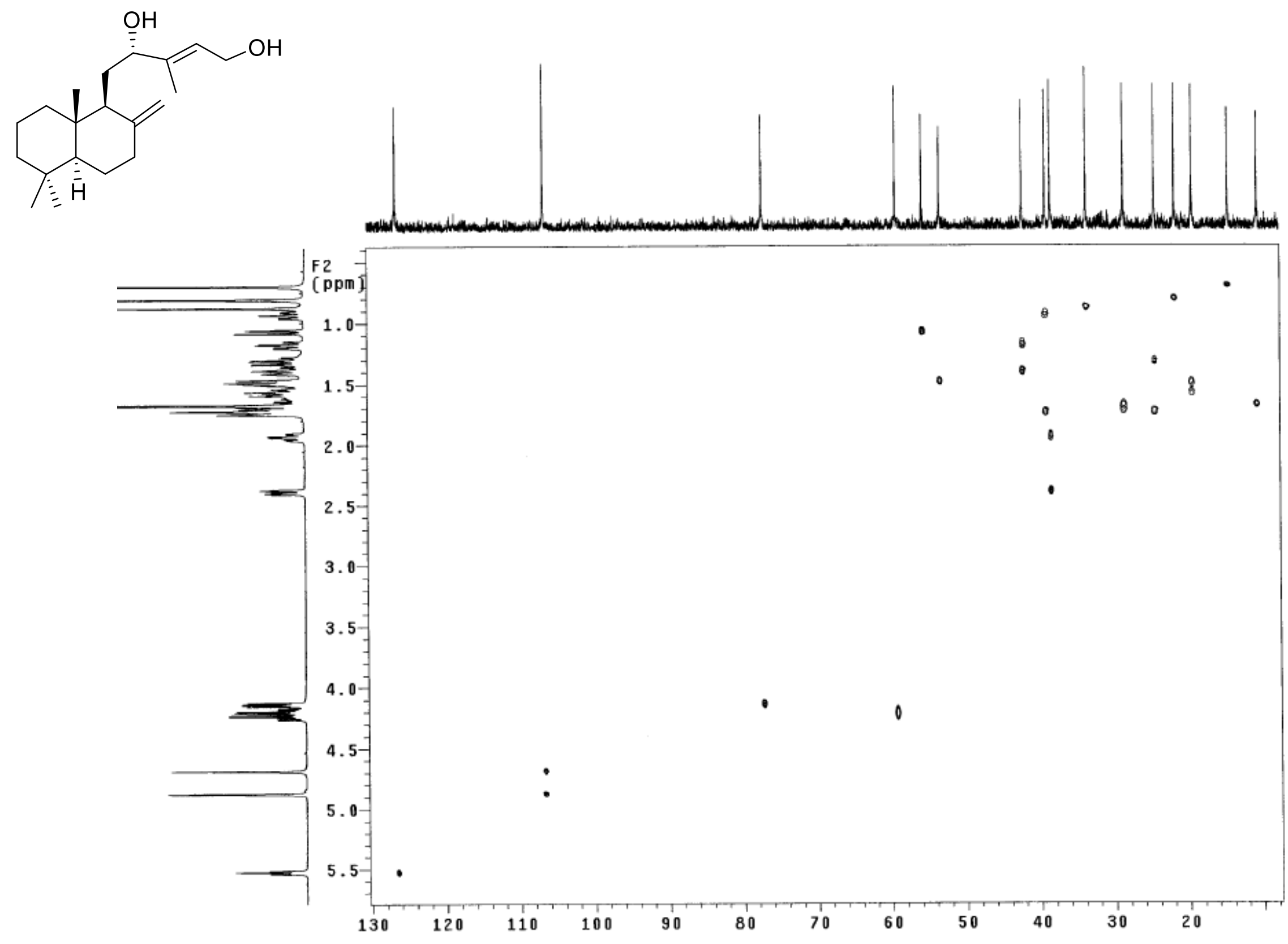

Figure S10. HSQC spectrum of compound $2\left(\mathrm{CDCl}_{3}\right)$. 


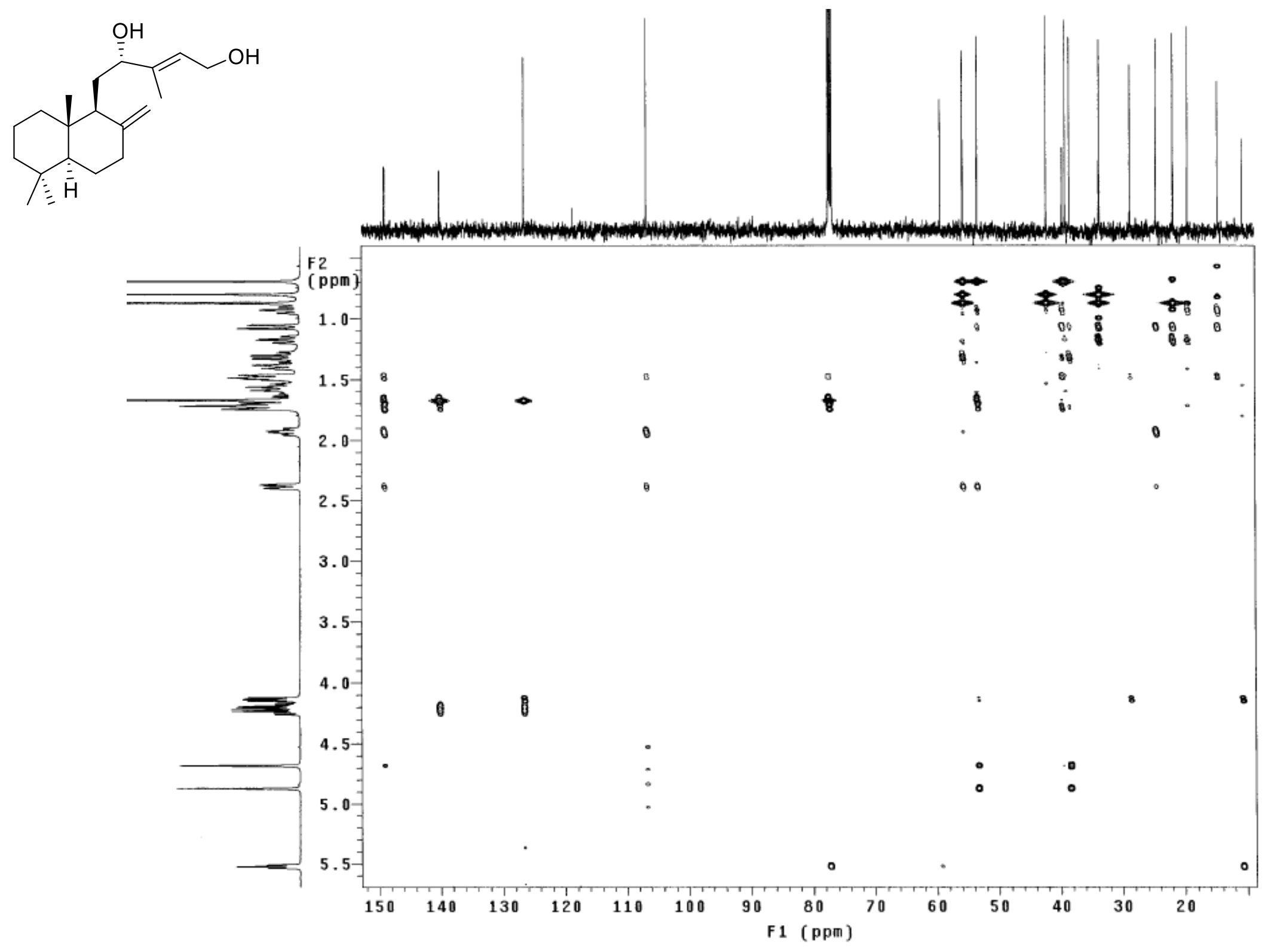

Figure S11. HMBC spectrum of compound $2\left(\mathrm{CDCl}_{3}\right)$. 

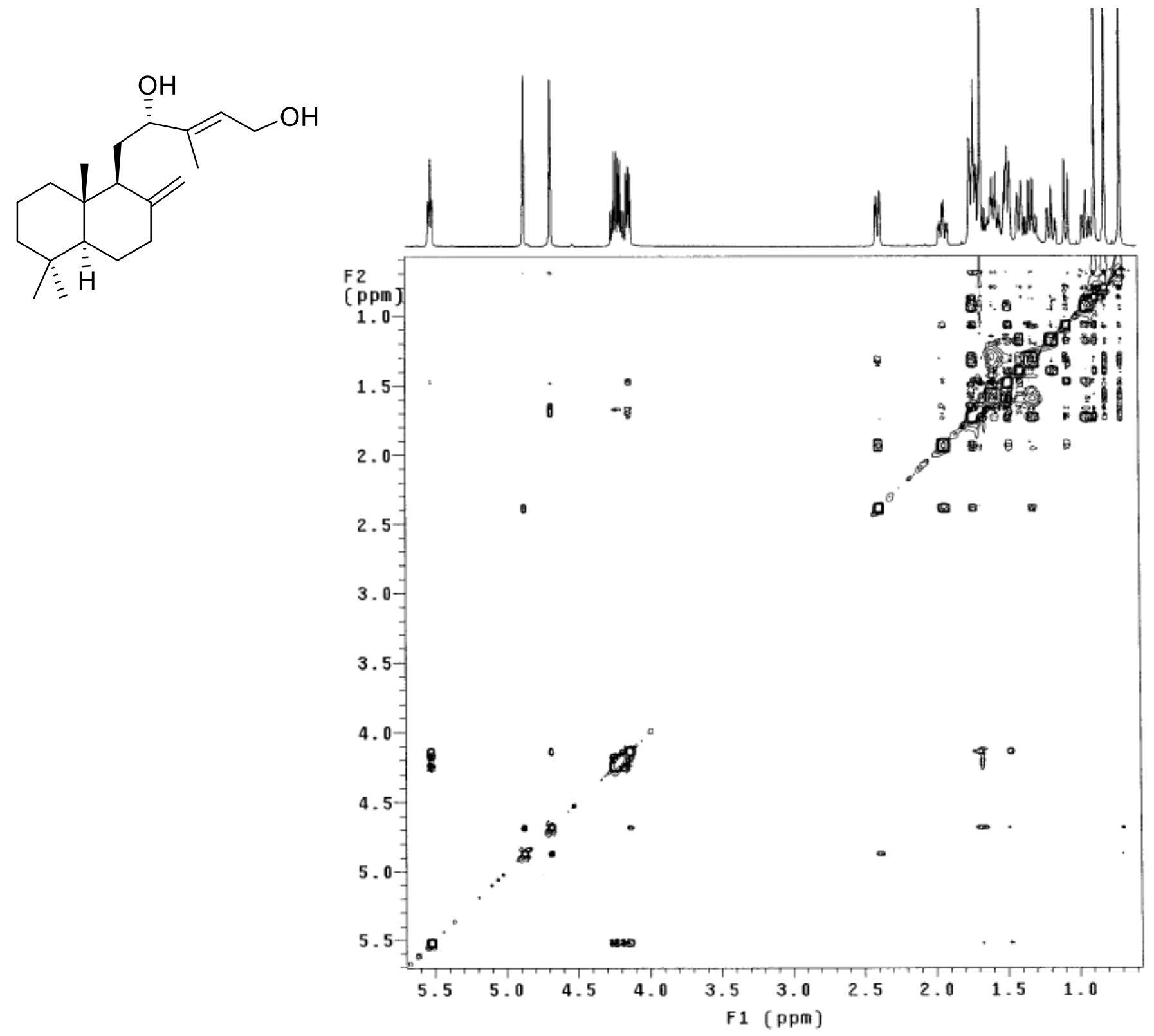

Figure S12. NOESY spectrum of compound $2\left(\mathrm{CDCl}_{3}\right)$. 


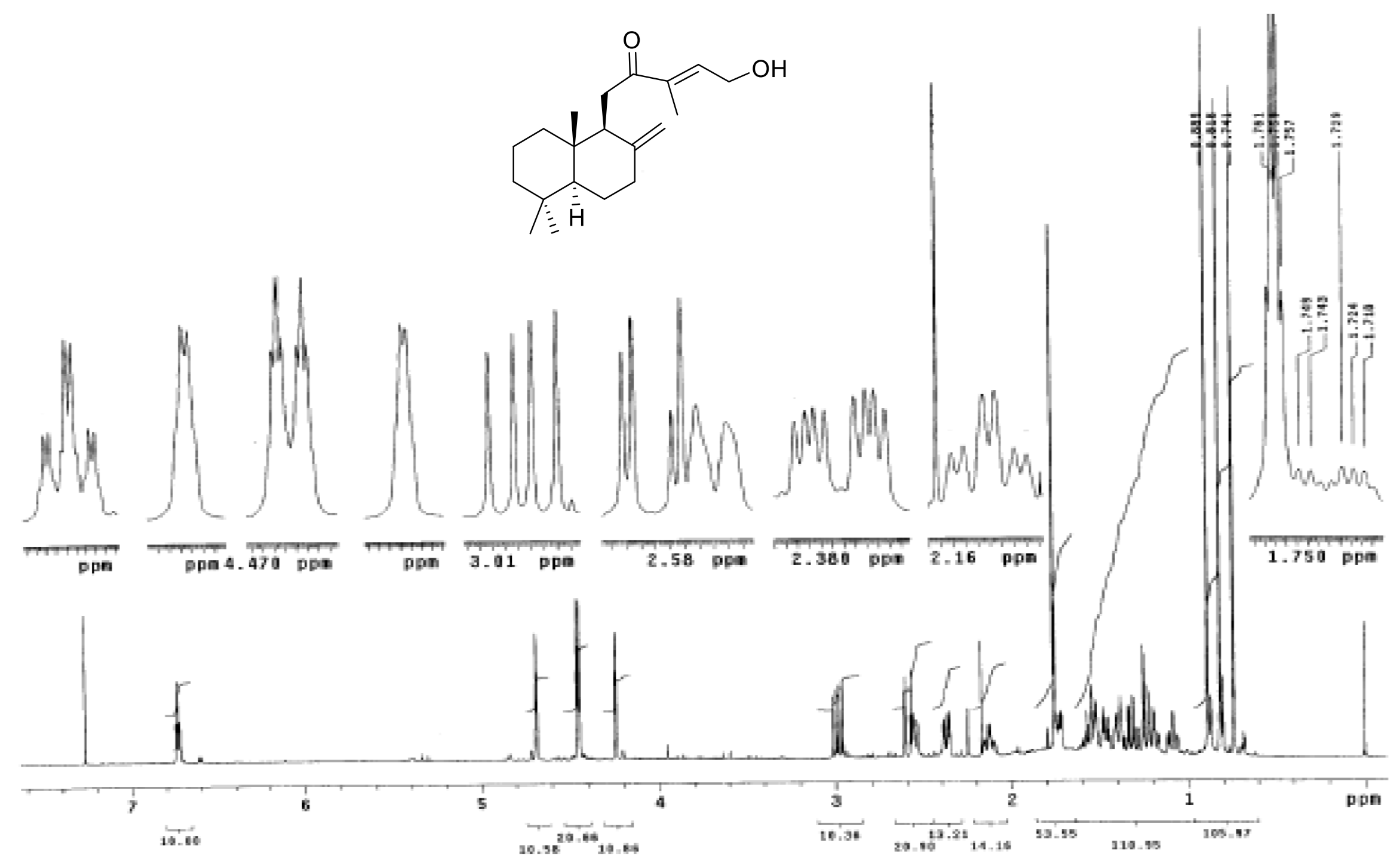

Figure S13. ${ }^{1} \mathrm{H}$ NMR spectrum of compound $3\left(500 \mathrm{MHz}, \mathrm{CDCl}_{3}\right)$. 


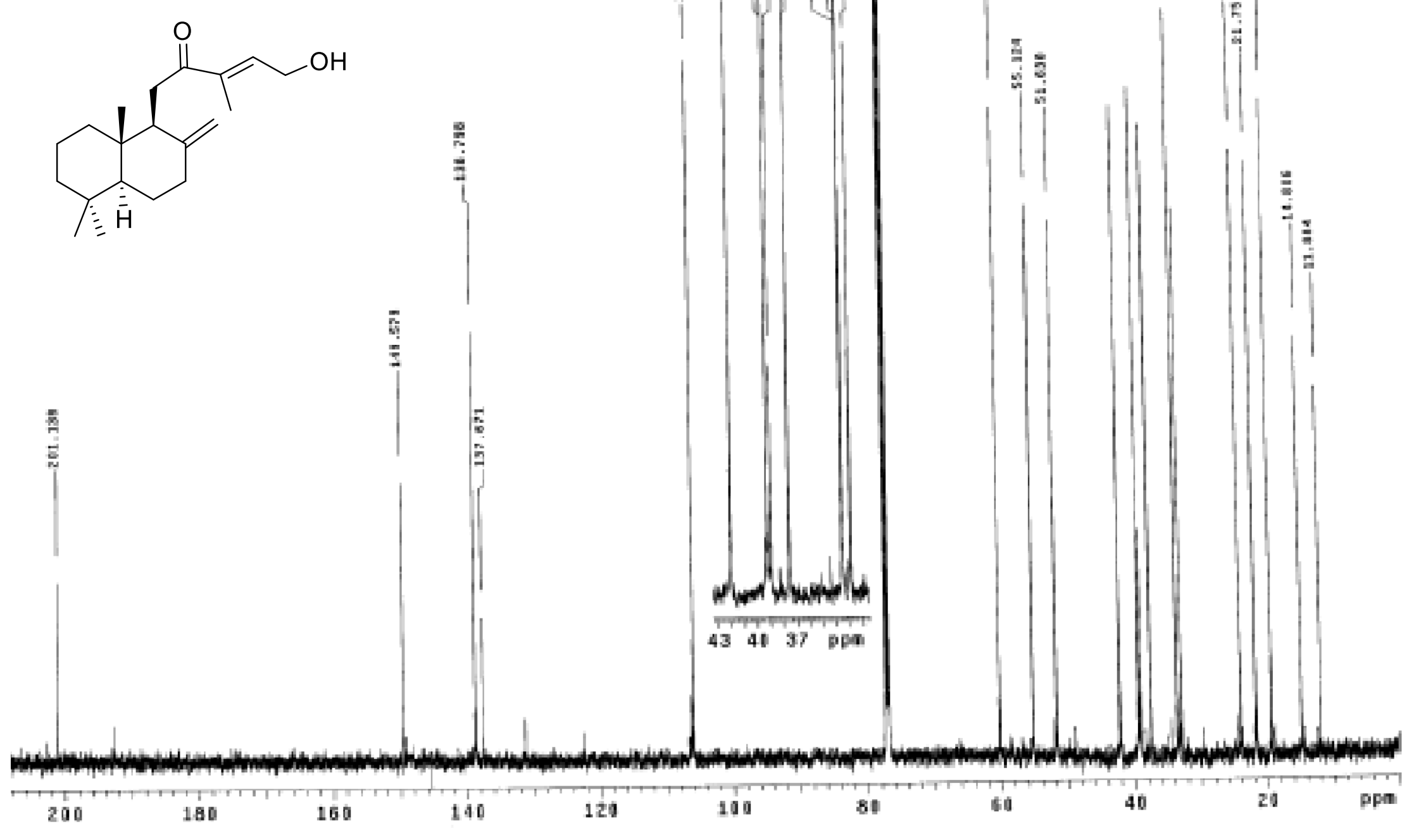

Figure S14. ${ }^{13} \mathrm{C}$ NMR spectrum of compound $3\left(125 \mathrm{MHz}, \mathrm{CDCl}_{3}\right)$. 


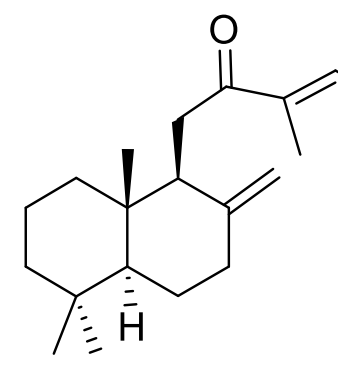

$\mathrm{OH}$

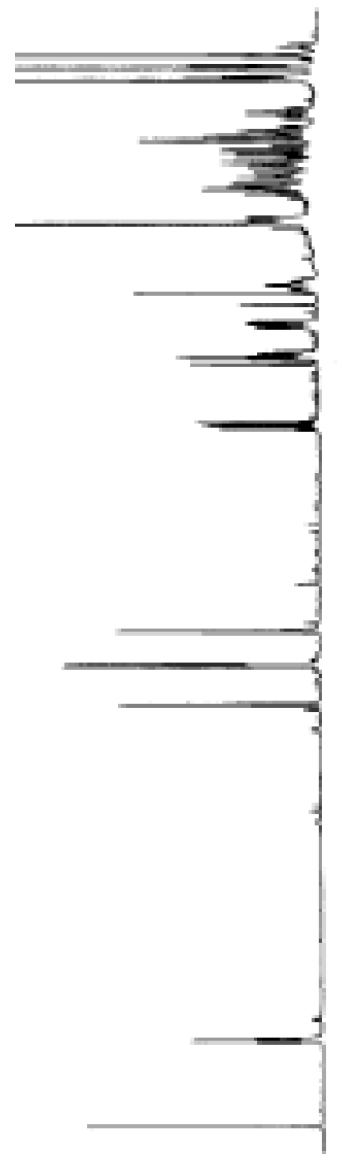

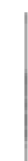
F2

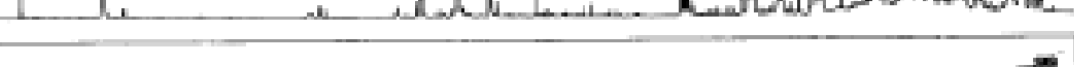

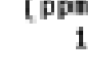

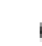

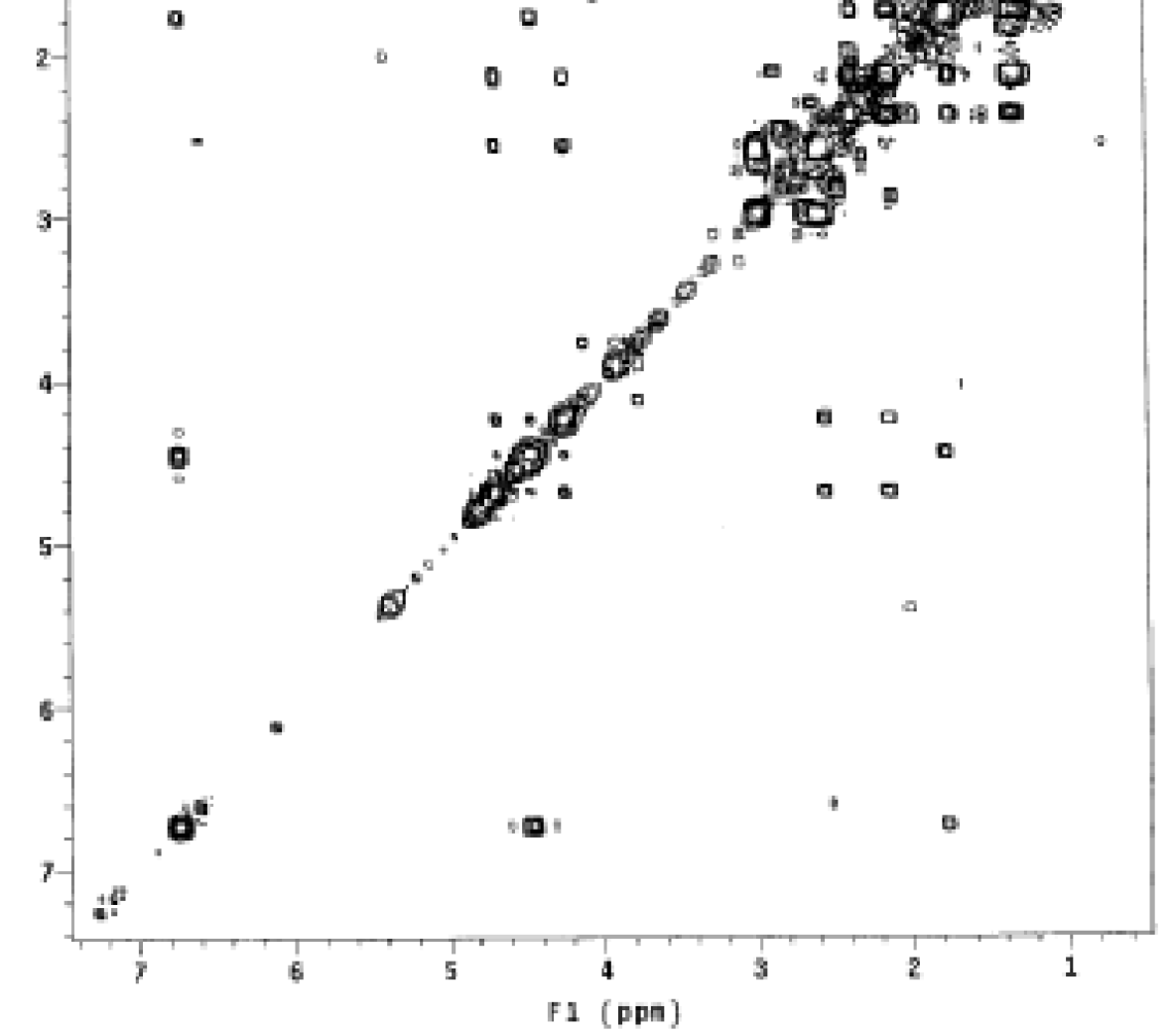

Figure S15. COSY spectrum of compound $3\left(\mathrm{CDCl}_{3}\right)$. 

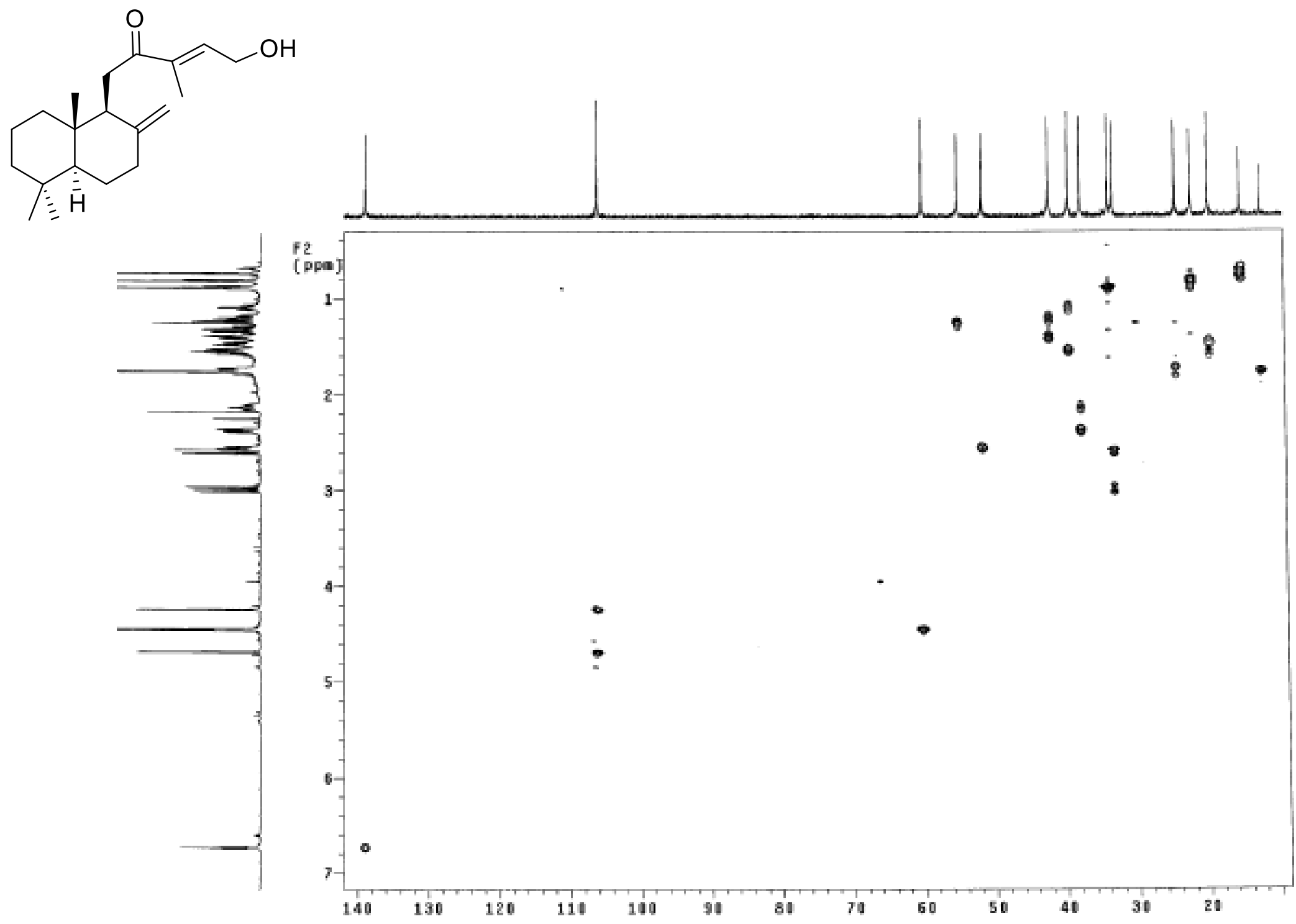

Figure S16. HSQC spectrum of compound $\mathbf{3}\left(\mathrm{CDCl}_{3}\right)$. 


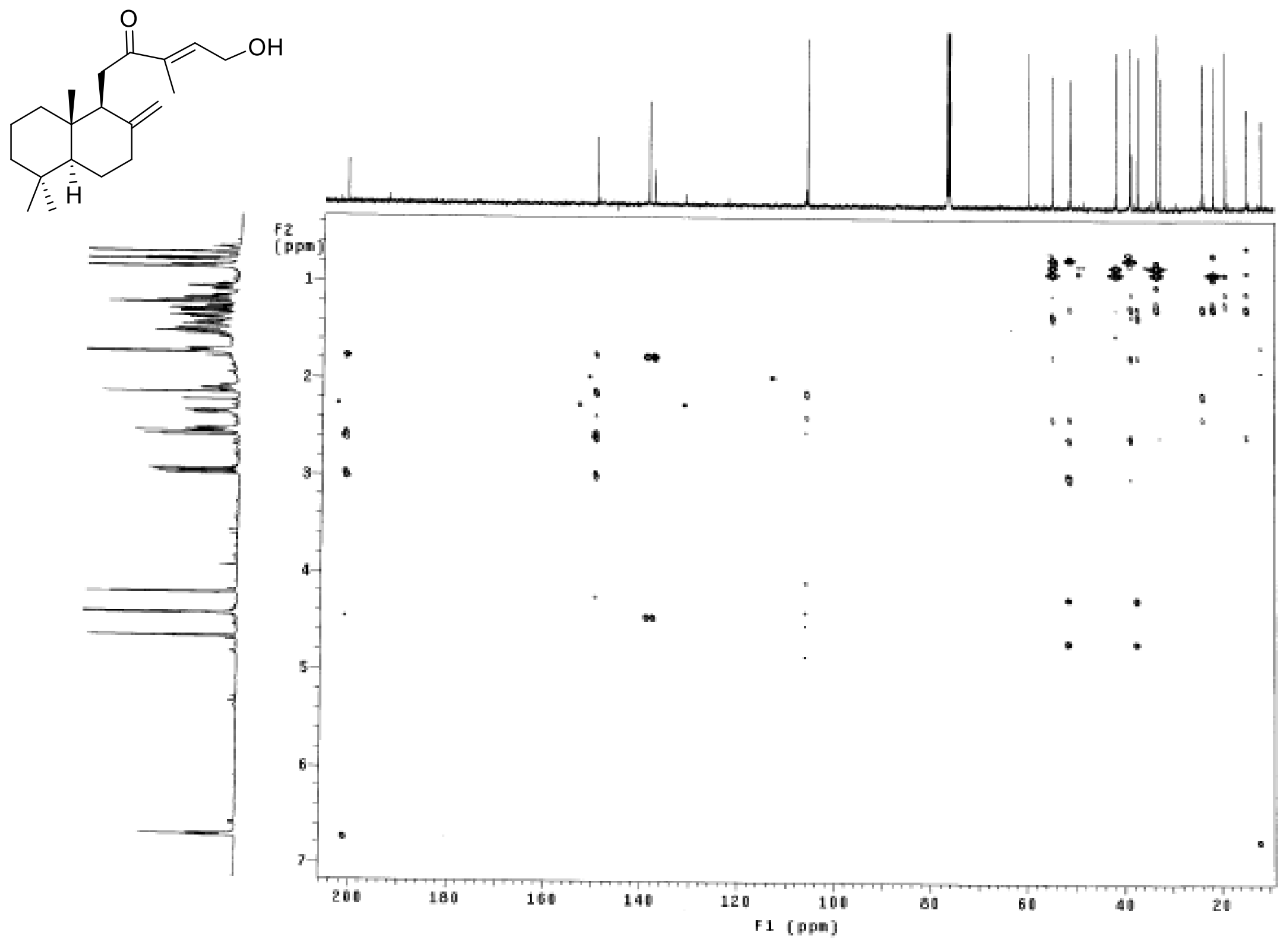

Figure S17. $\mathrm{HMBC}$ spectrum of compound $\mathbf{3}\left(\mathrm{CDCl}_{3}\right)$. 


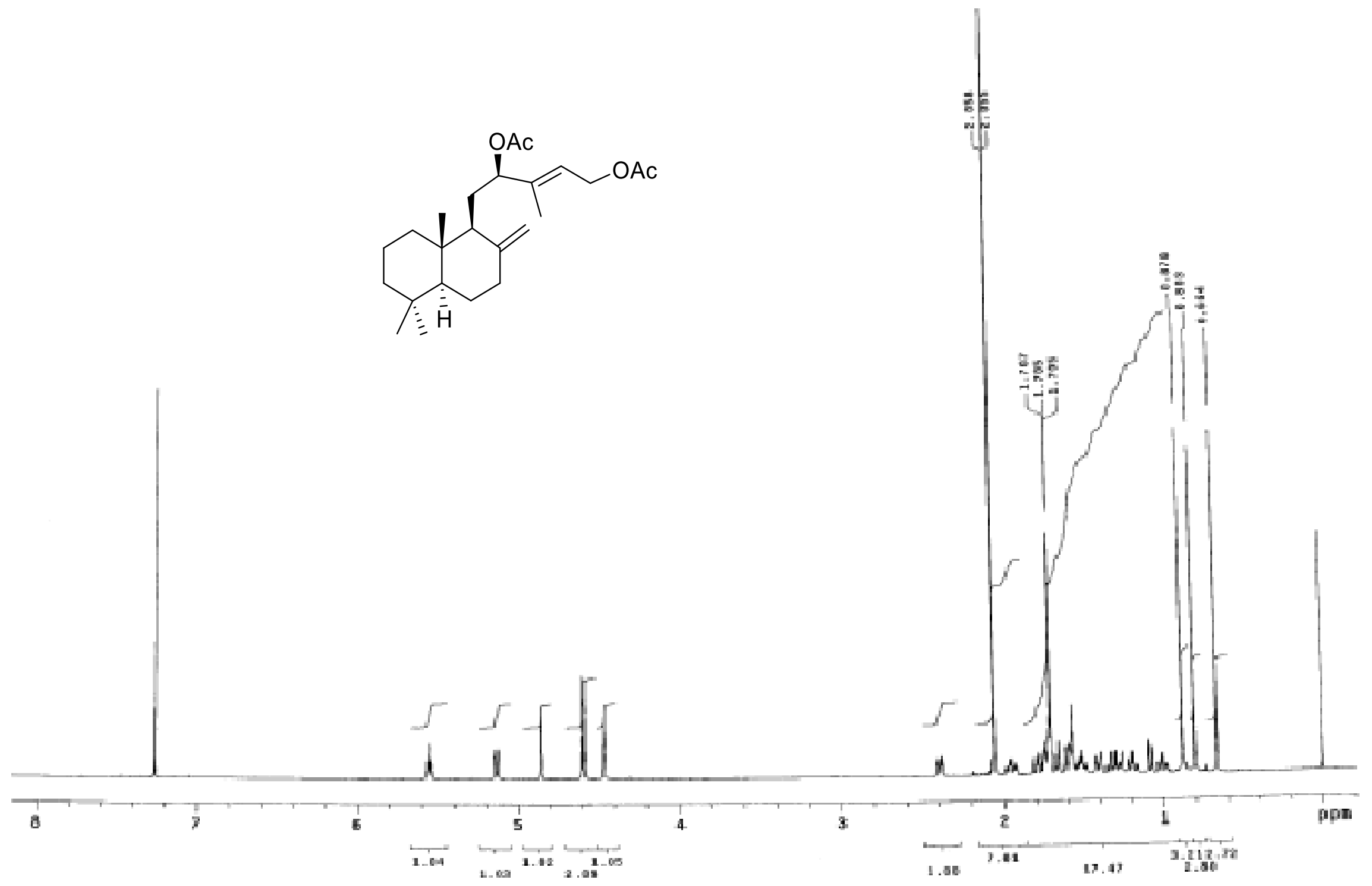

Figure S18. ${ }^{1} \mathrm{H}$ NMR spectrum of compound $1 \mathbf{a}\left(500 \mathrm{MHz}, \mathrm{CDCl}_{3}\right)$. 


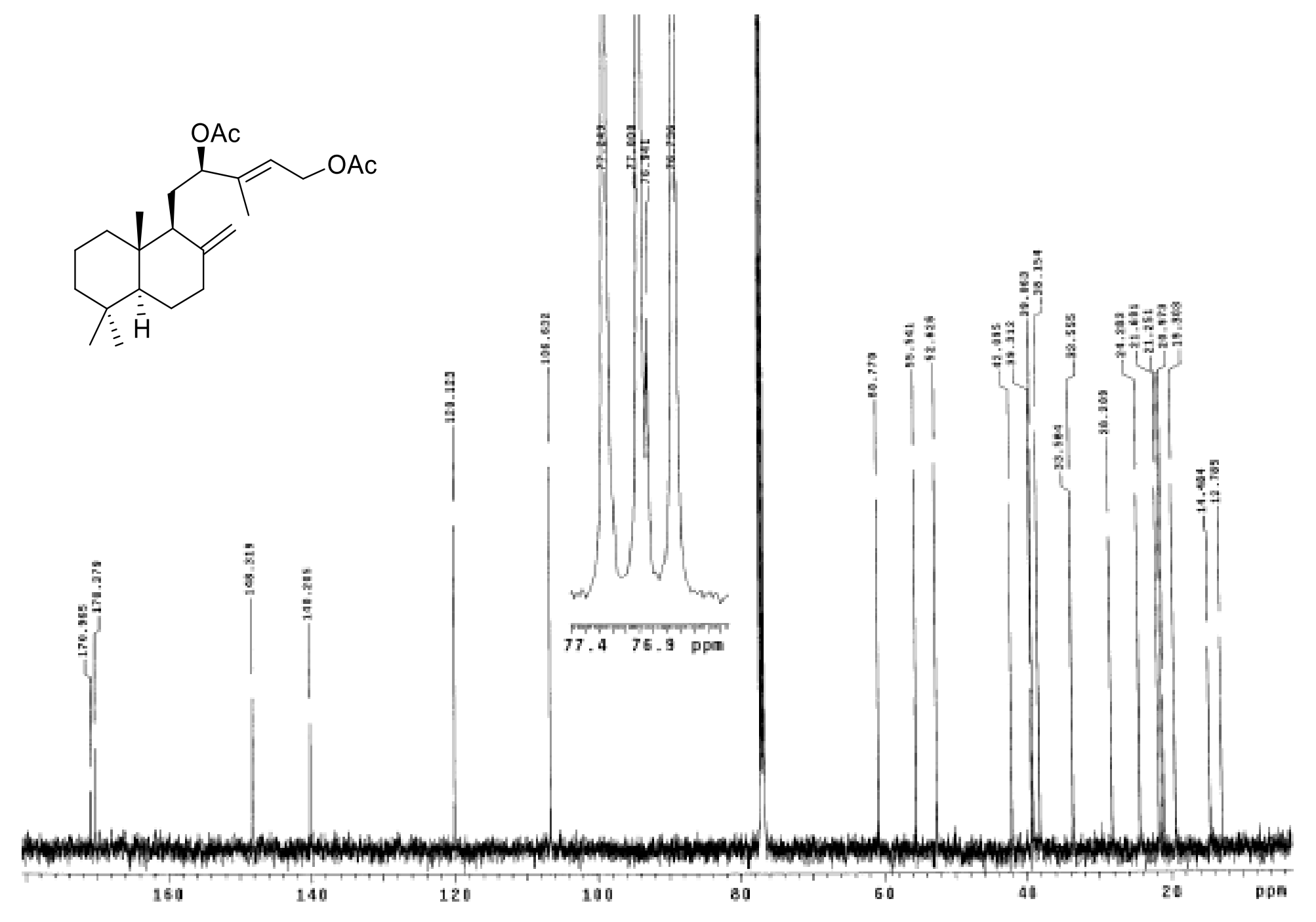

Figure S19. ${ }^{13} \mathrm{C}$ NMR spectrum of compound $1 \mathbf{a}\left(125 \mathrm{MHz}, \mathrm{CDCl}_{3}\right)$. 


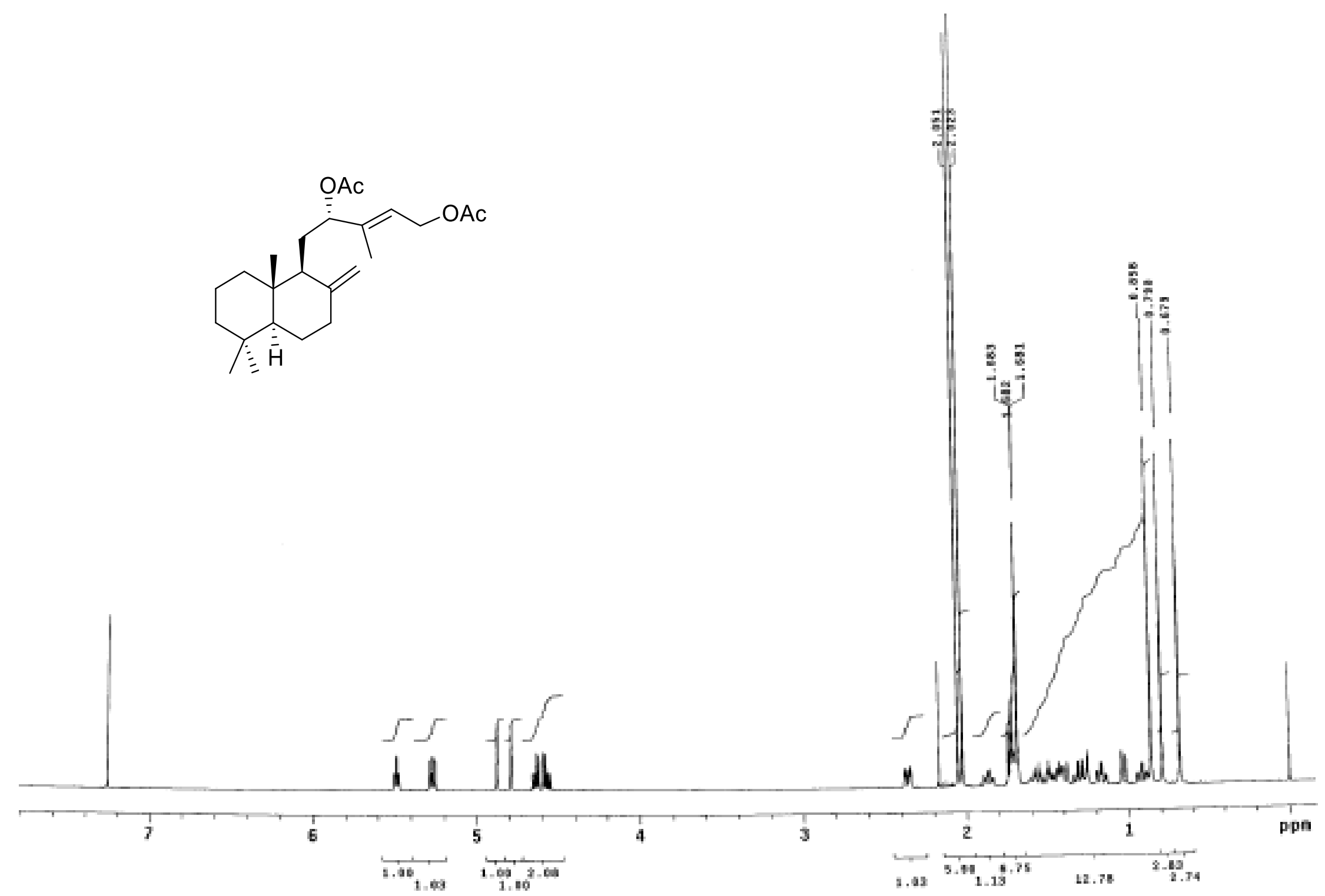

Figure S20. ${ }^{1} \mathrm{H}$ NMR spectrum of compound $2 \mathbf{a}\left(500 \mathrm{MHz}, \mathrm{CDCl}_{3}\right)$. 

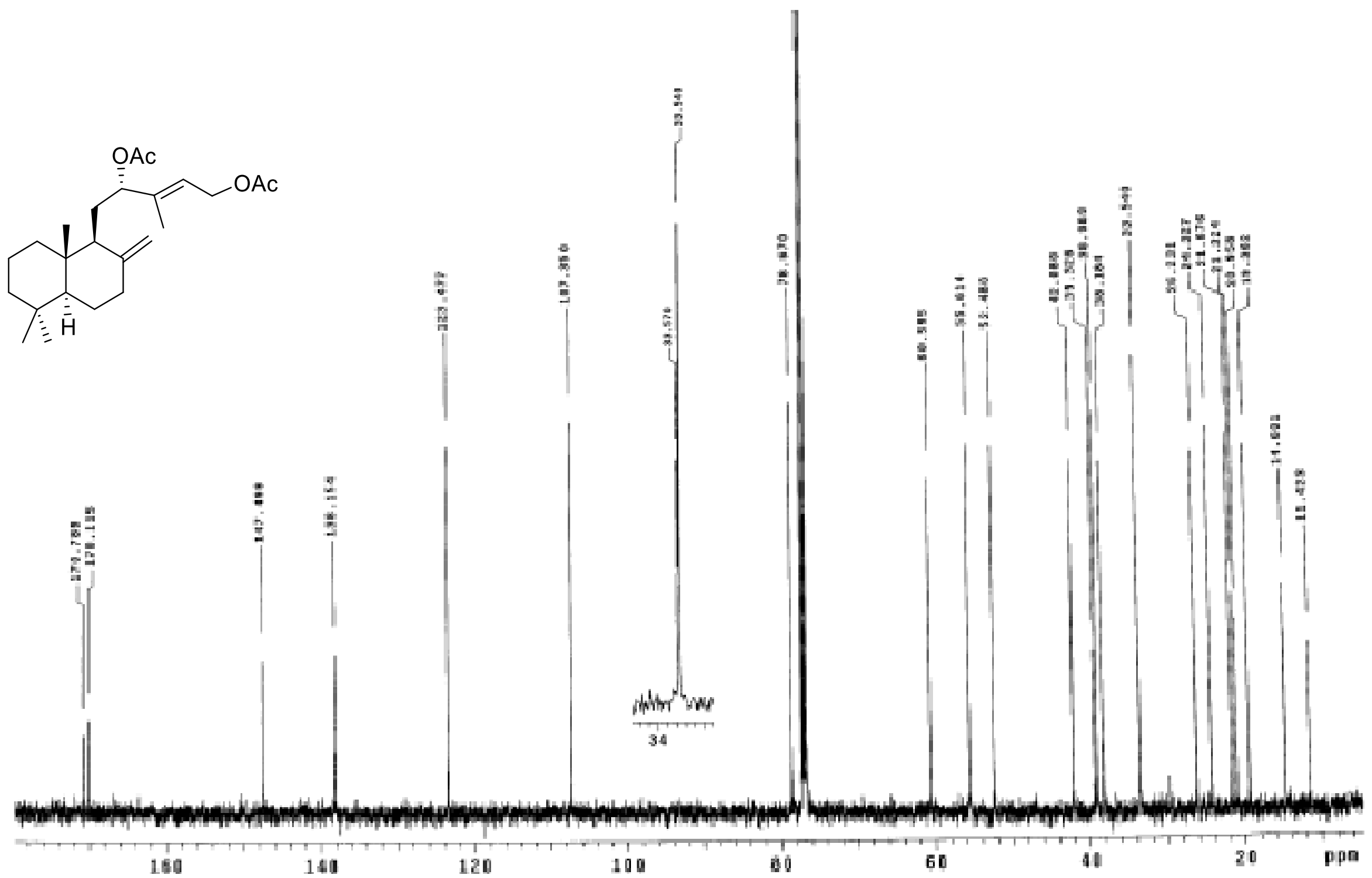

Figure S21. ${ }^{13} \mathrm{C}$ NMR spectrum of compound $\mathbf{2 a}\left(125 \mathrm{MHz}, \mathrm{CDCl}_{3}\right)$. 


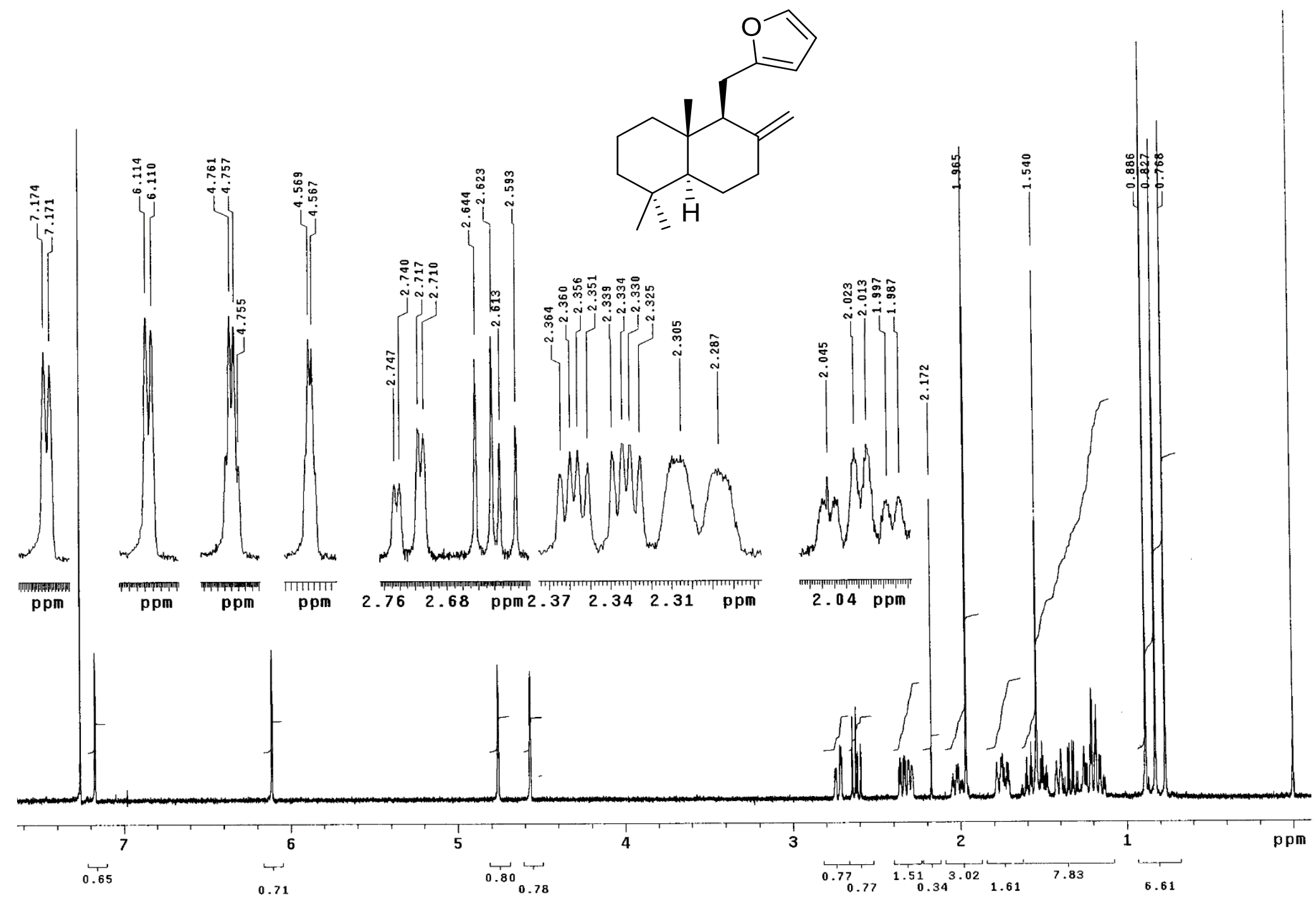

Figure S22. ${ }^{1} \mathrm{H}$ NMR spectrum of compound 3a $\left(500 \mathrm{MHz}, \mathrm{CDCl}_{3}\right)$. 


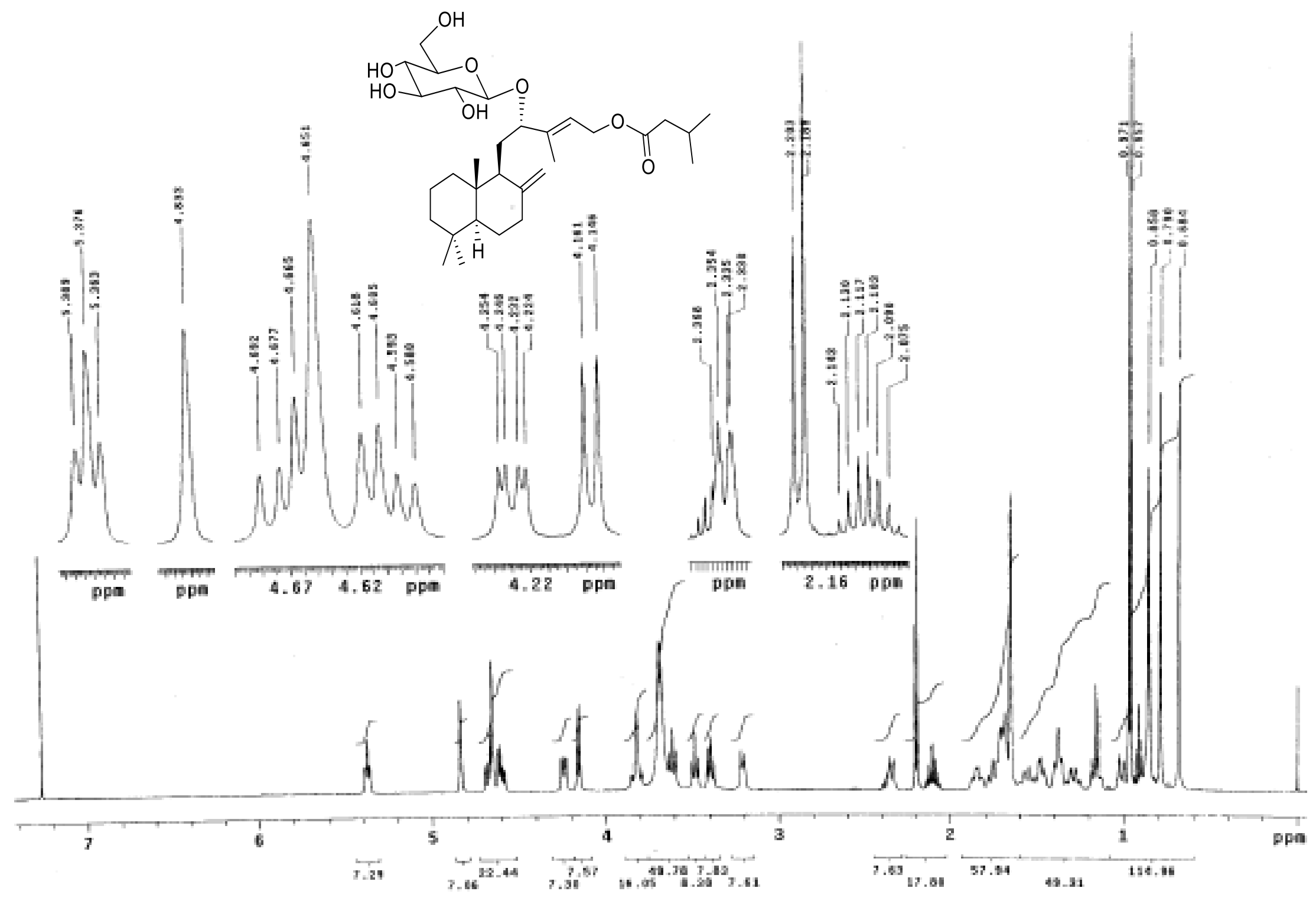

Figure S23. ${ }^{1} \mathrm{H}$ NMR spectrum of compound 4 (500 $\left.\mathrm{MHz}, \mathrm{CDCl}_{3}\right)$. 


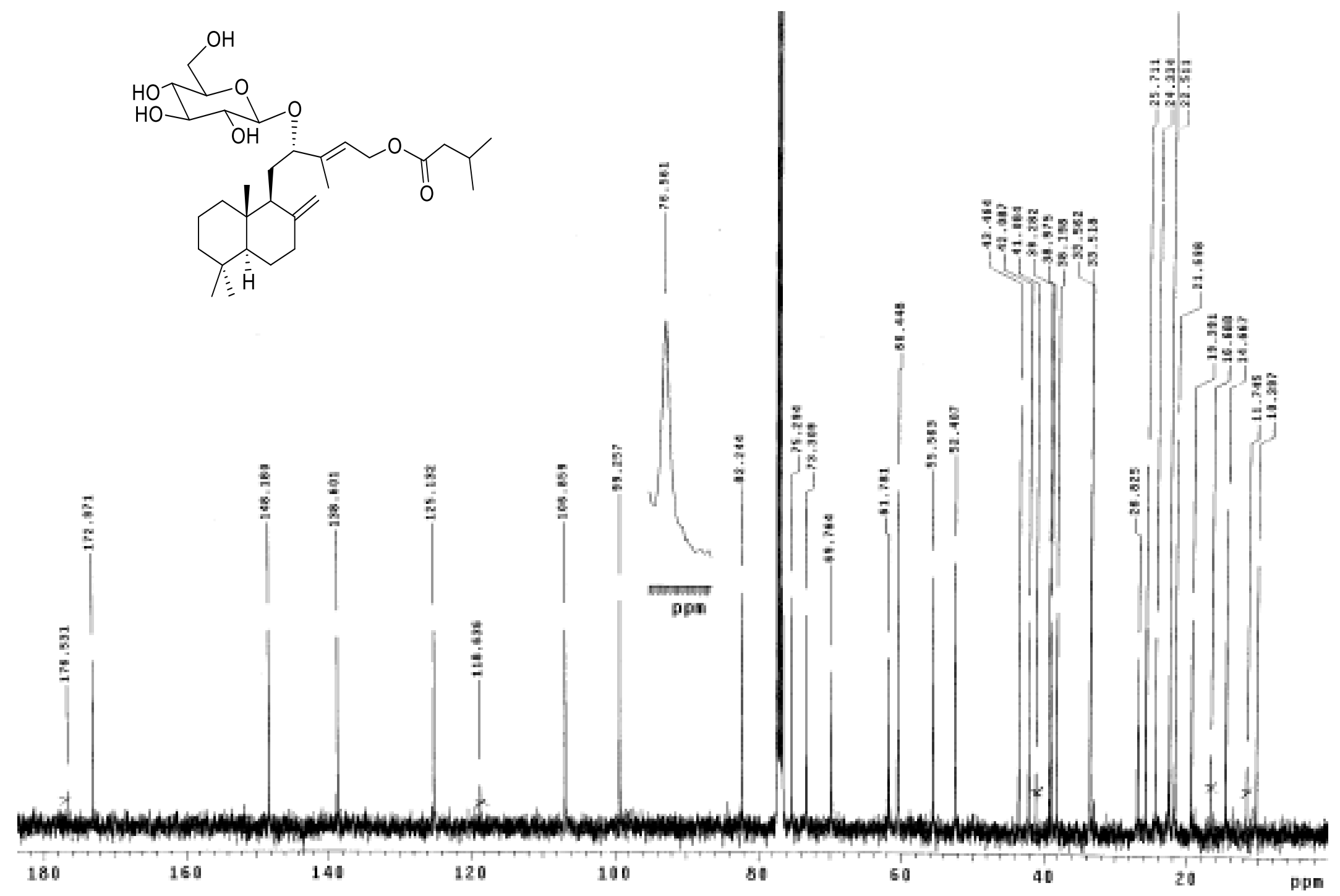

Figure S24. ${ }^{13} \mathrm{C}$ NMR spectrum of compound $4\left(125 \mathrm{MHz}, \mathrm{CDCl}_{3}\right)$. 


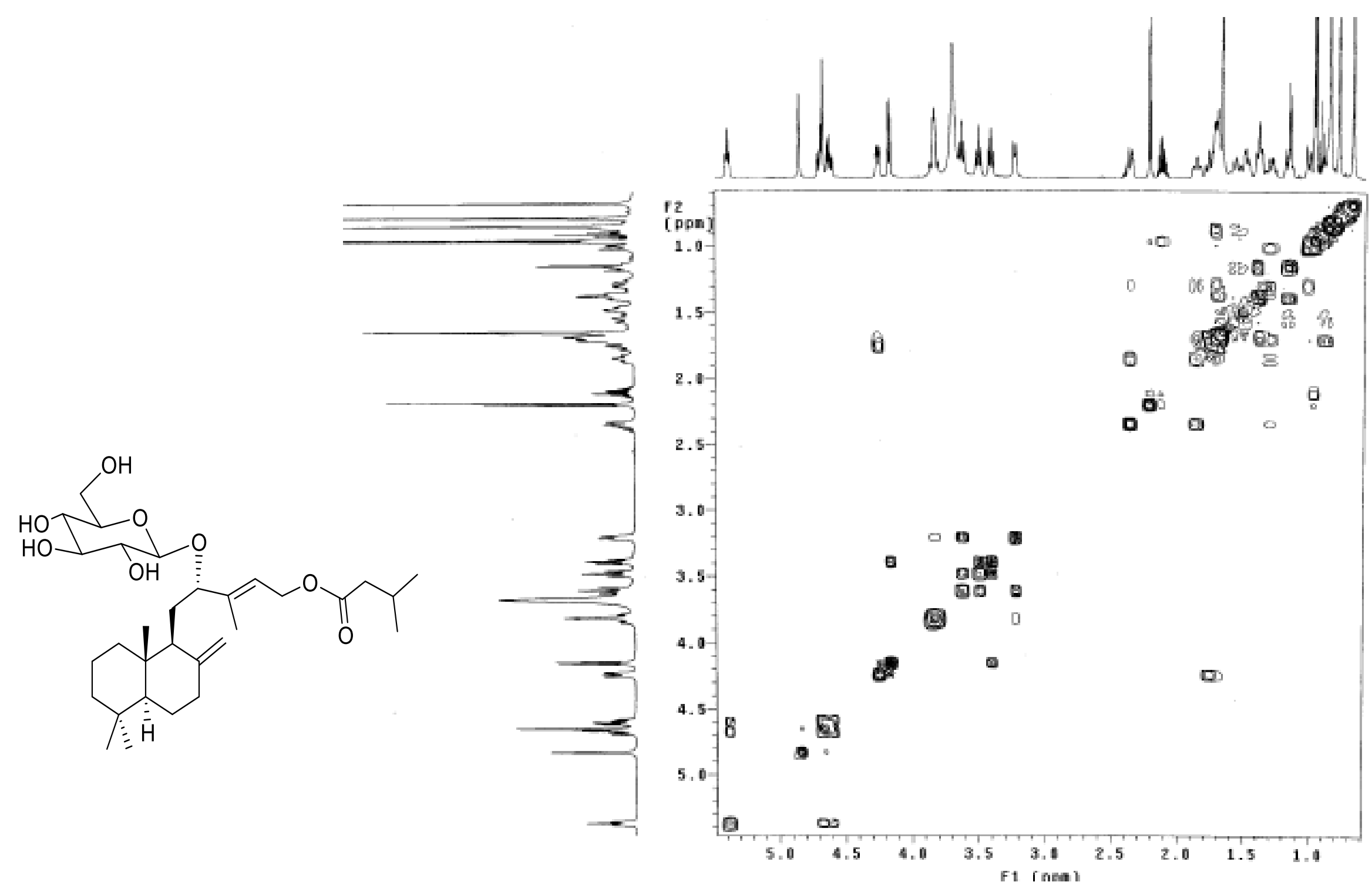

Figure S25. COSY spectrum of compound $4\left(\mathrm{CDCl}_{3}\right)$. 


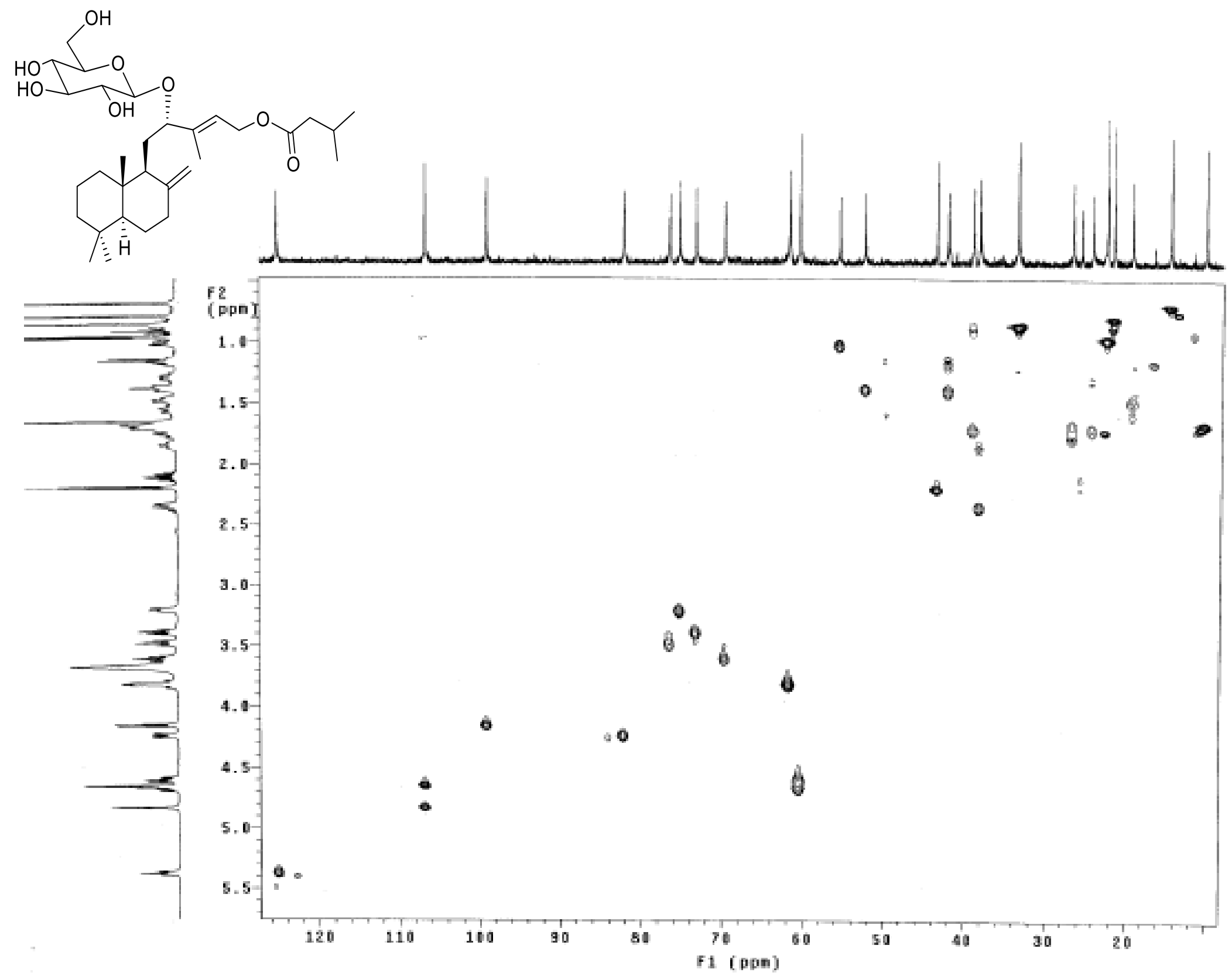

Figure S26. HSQC spectrum of compound $4\left(\mathrm{CDCl}_{3}\right)$. 


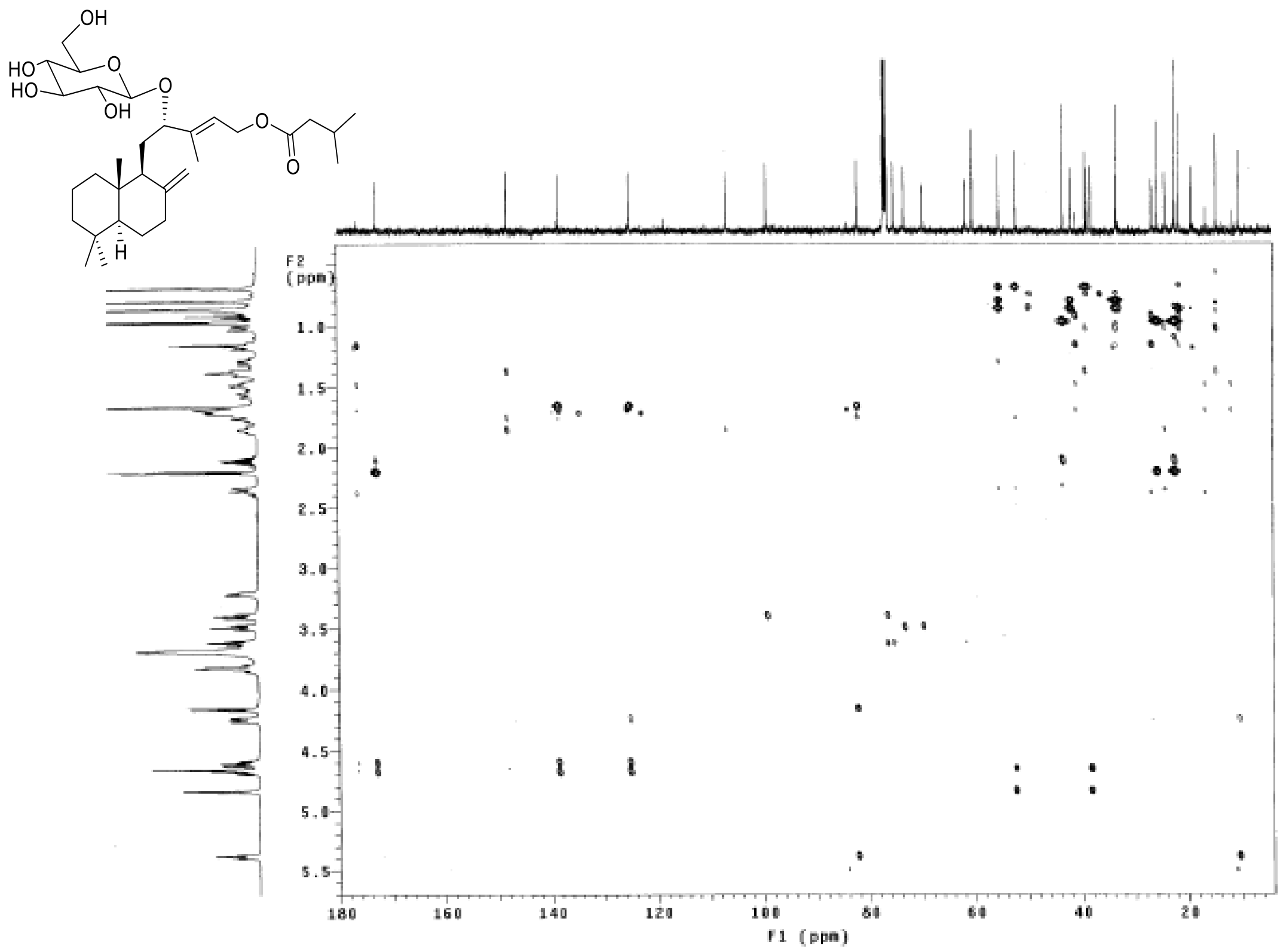

Figure S27. HMBC spectrum of compound $4\left(\mathrm{CDCl}_{3}\right)$. 


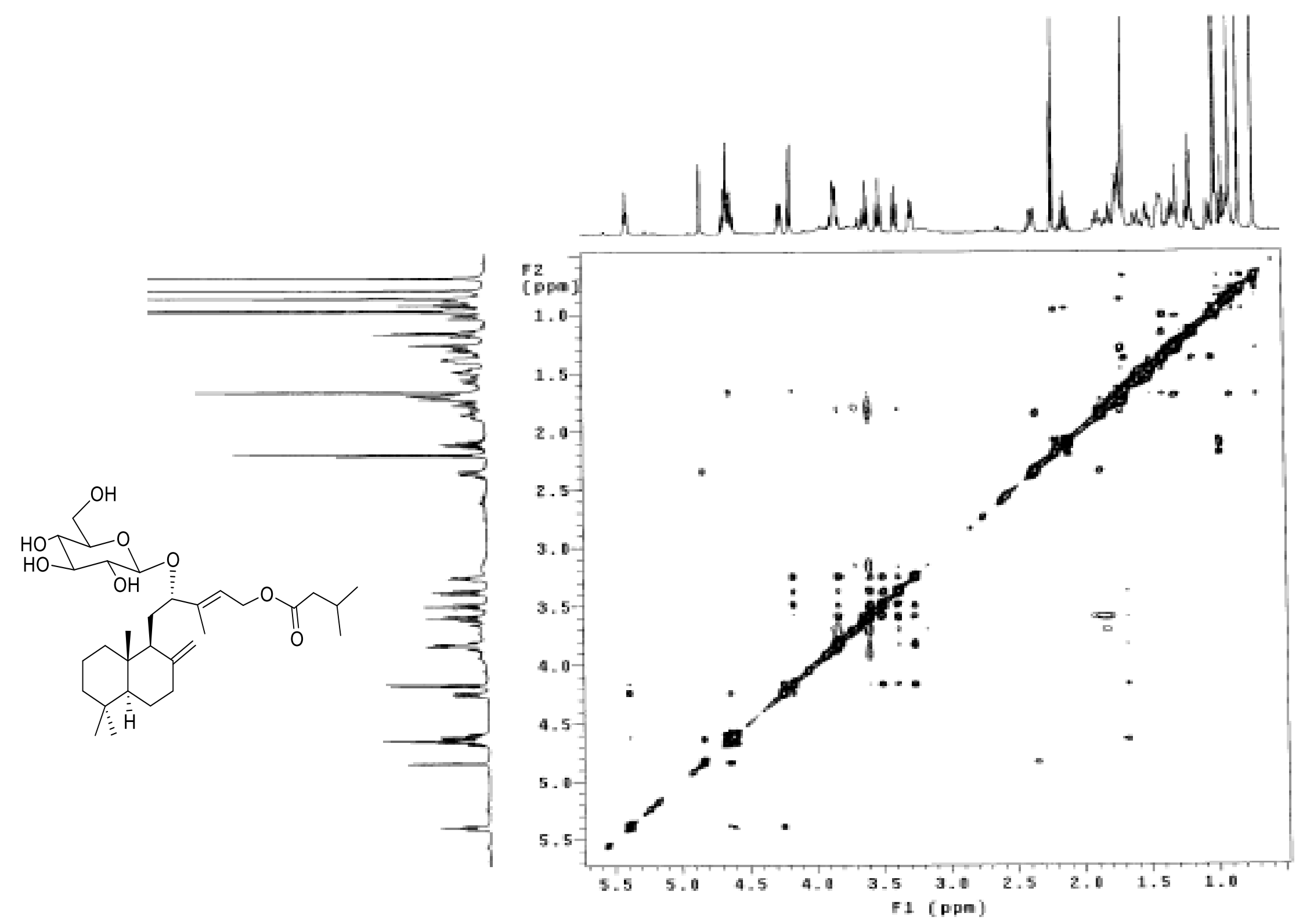

Figure S28. NOESY spectrum of compound $4\left(\mathrm{CDCl}_{3}\right)$. 


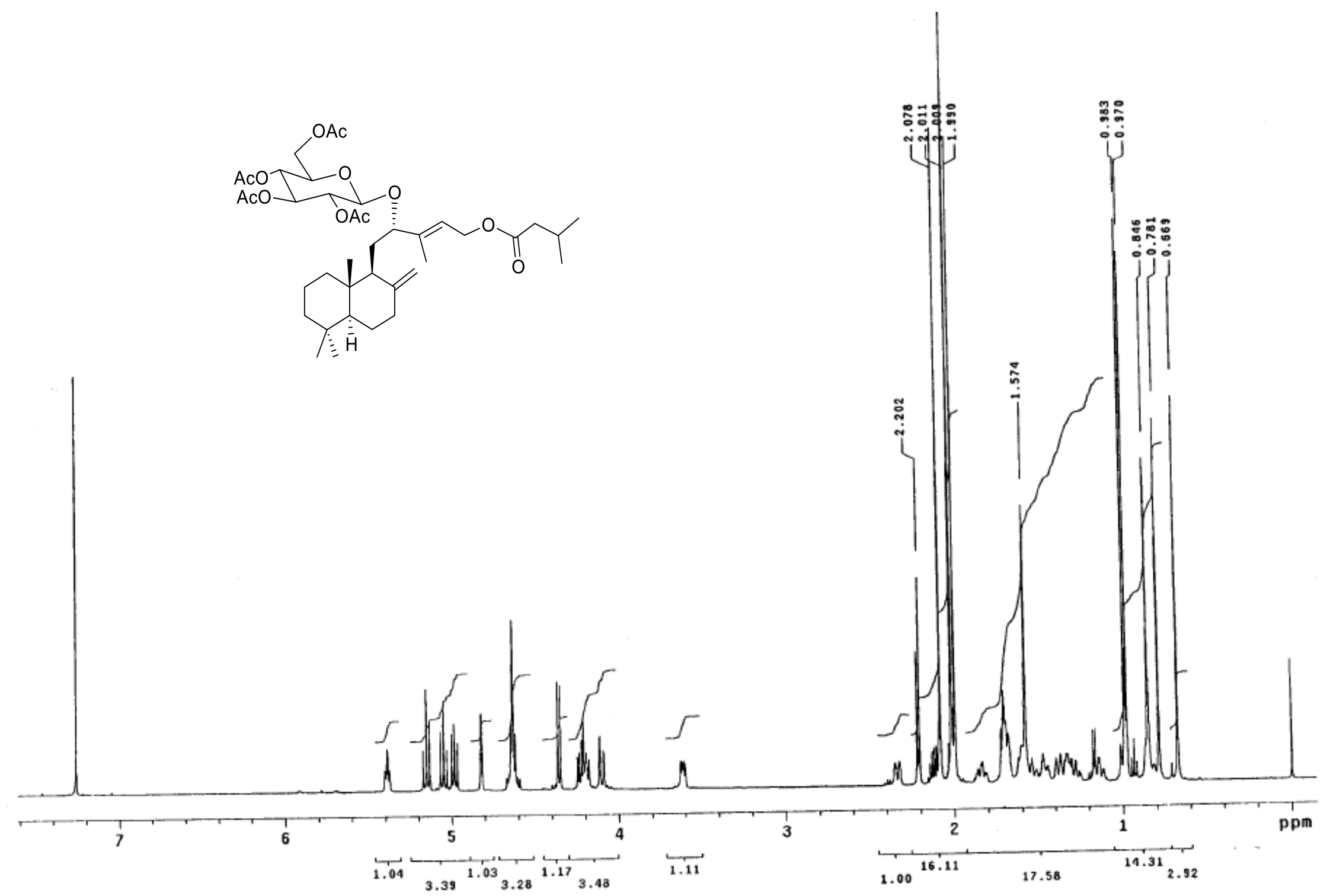

Figure S29. ${ }^{1} \mathrm{H}$ NMR spectrum of compound $4 \mathbf{a}\left(500 \mathrm{MHz}, \mathrm{CDCl}_{3}\right)$. 


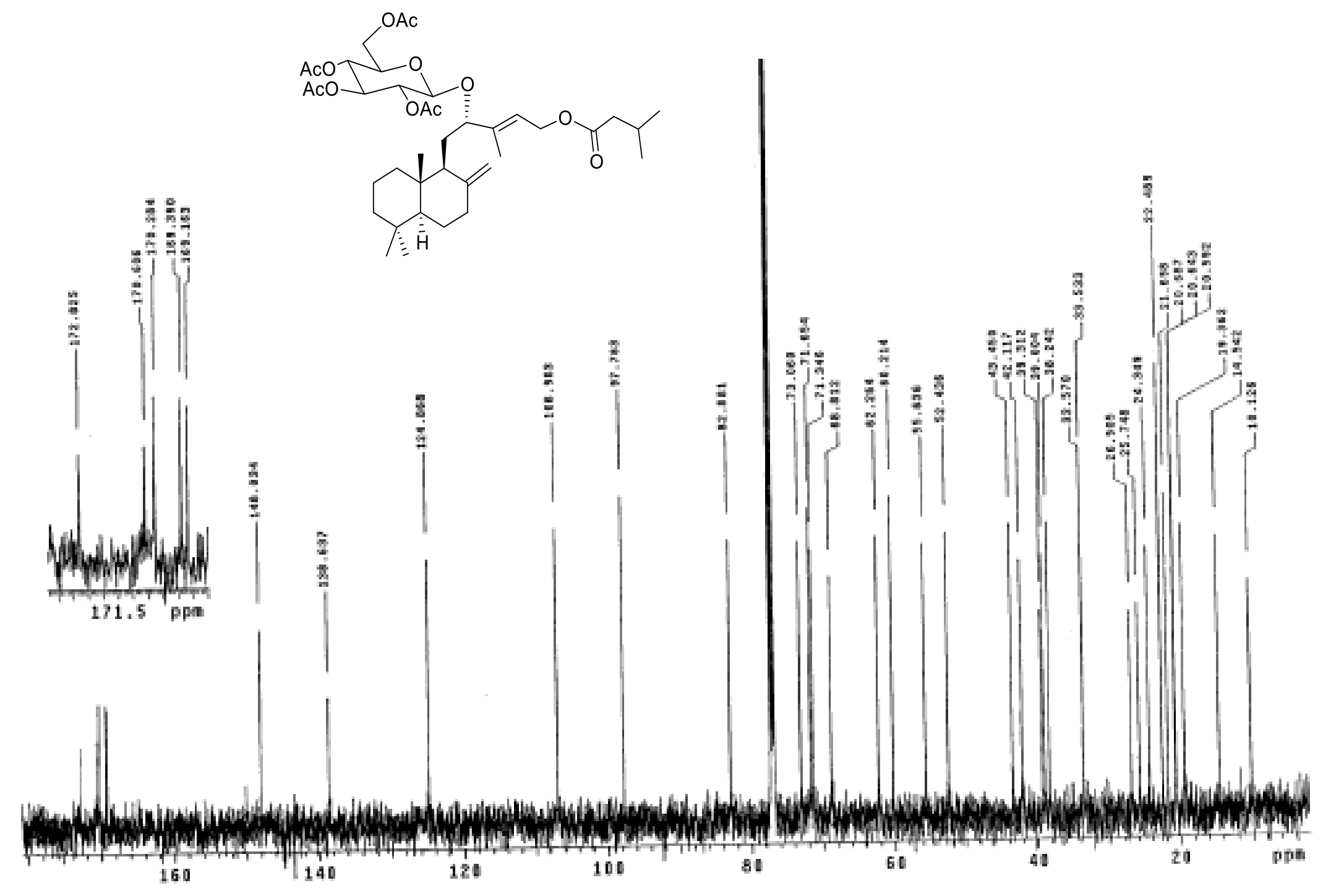

Figure S30. ${ }^{13} \mathrm{C}$ NMR spectrum of compound $\mathbf{4 a}\left(125 \mathrm{MHz}, \mathrm{CDCl}_{3}\right)$. 


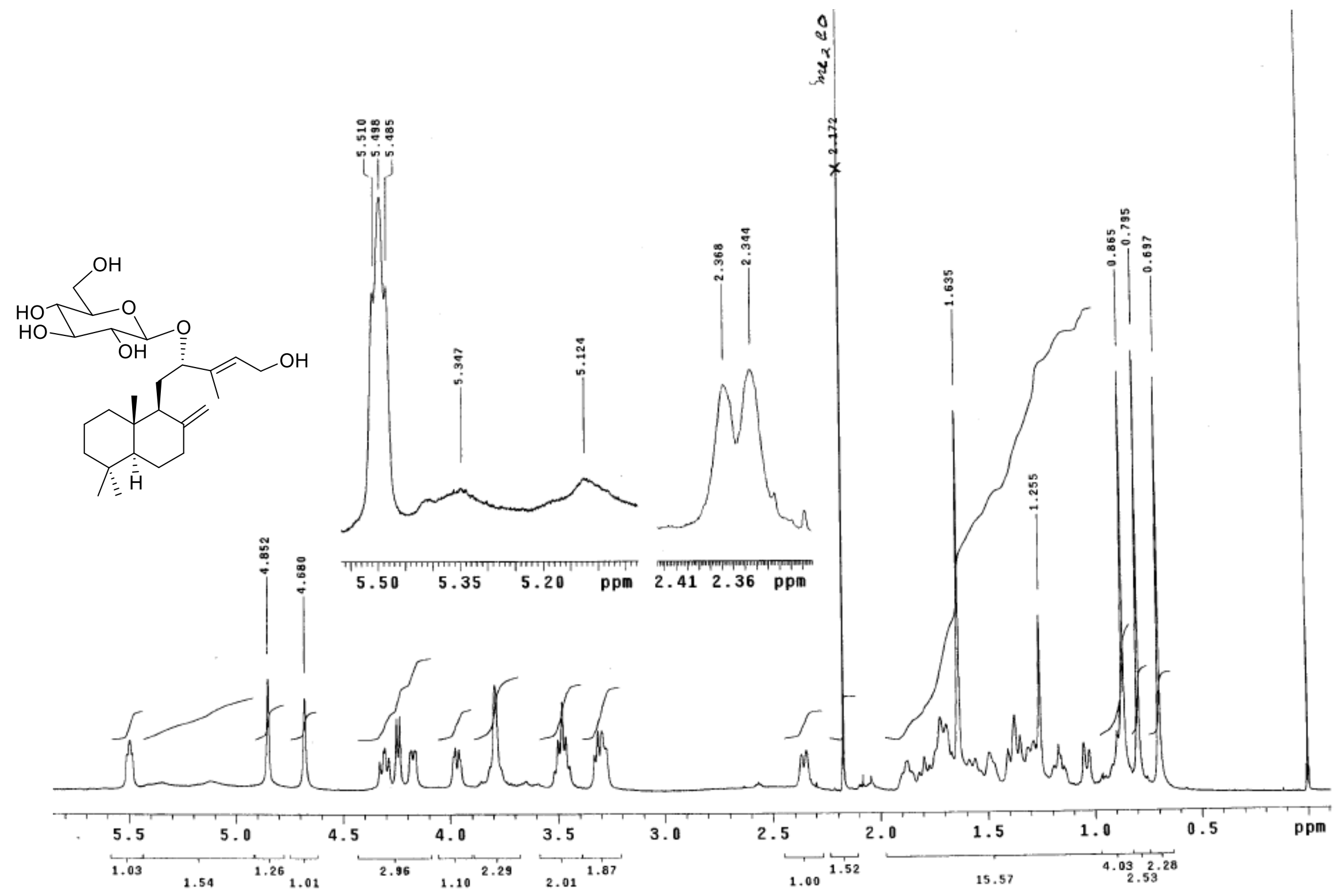

Figure S31. ${ }^{1} \mathrm{H}$ NMR spectrum of compound $5\left(500 \mathrm{MHz}, \mathrm{CDCl}_{3}\right)$. 


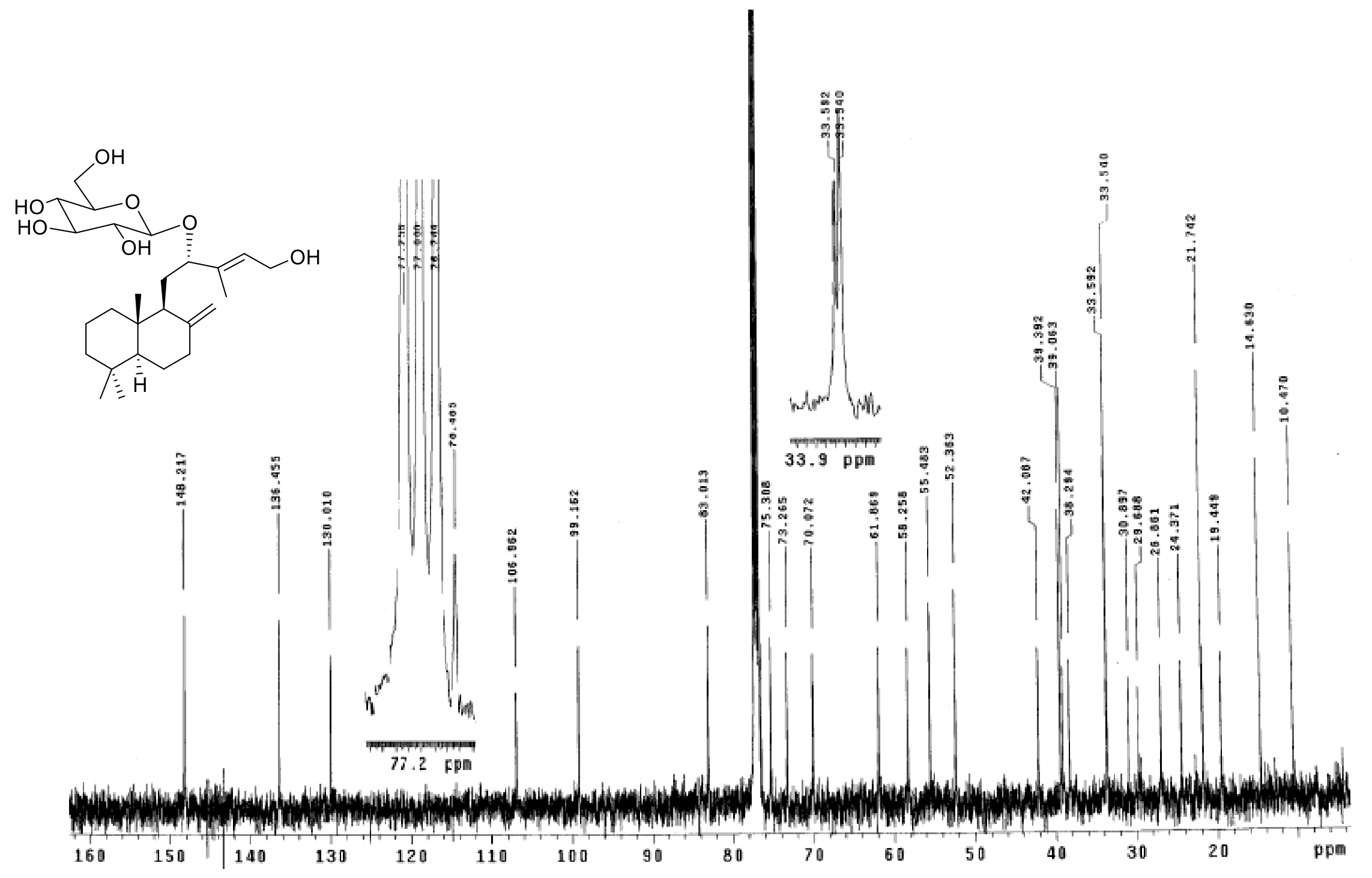

Figure S32. ${ }^{13} \mathrm{C} \mathrm{NMR}$ spectrum of compound $5\left(125 \mathrm{MHz}, \mathrm{CDCl}_{3}\right)$. 


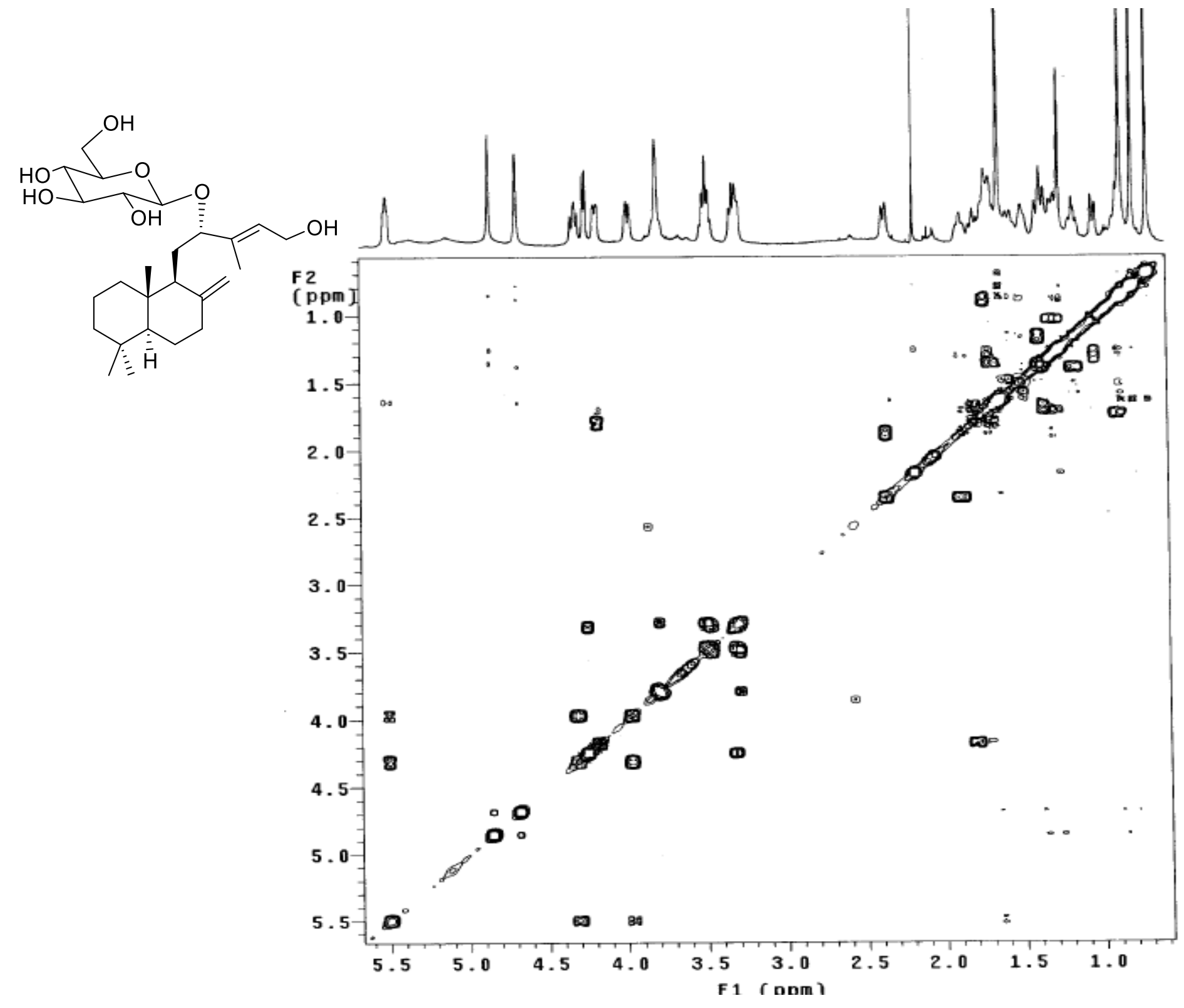

Figure S33. COSY spectrum of compound $\mathbf{5}\left(\mathrm{CDCl}_{3}\right)$. 


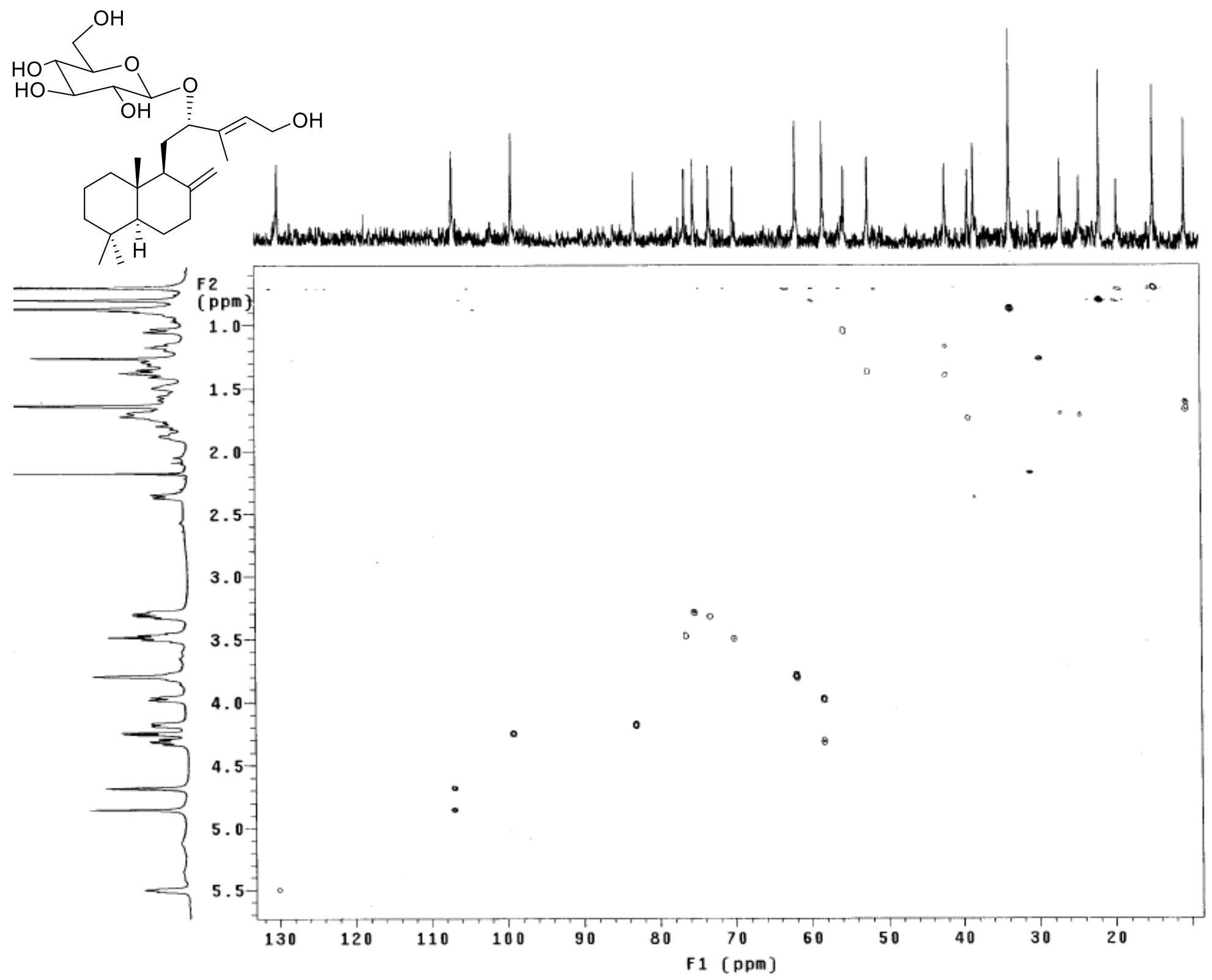

Figure S34. HSQC spectrum of compound $5\left(\mathrm{CDCl}_{3}\right)$. 


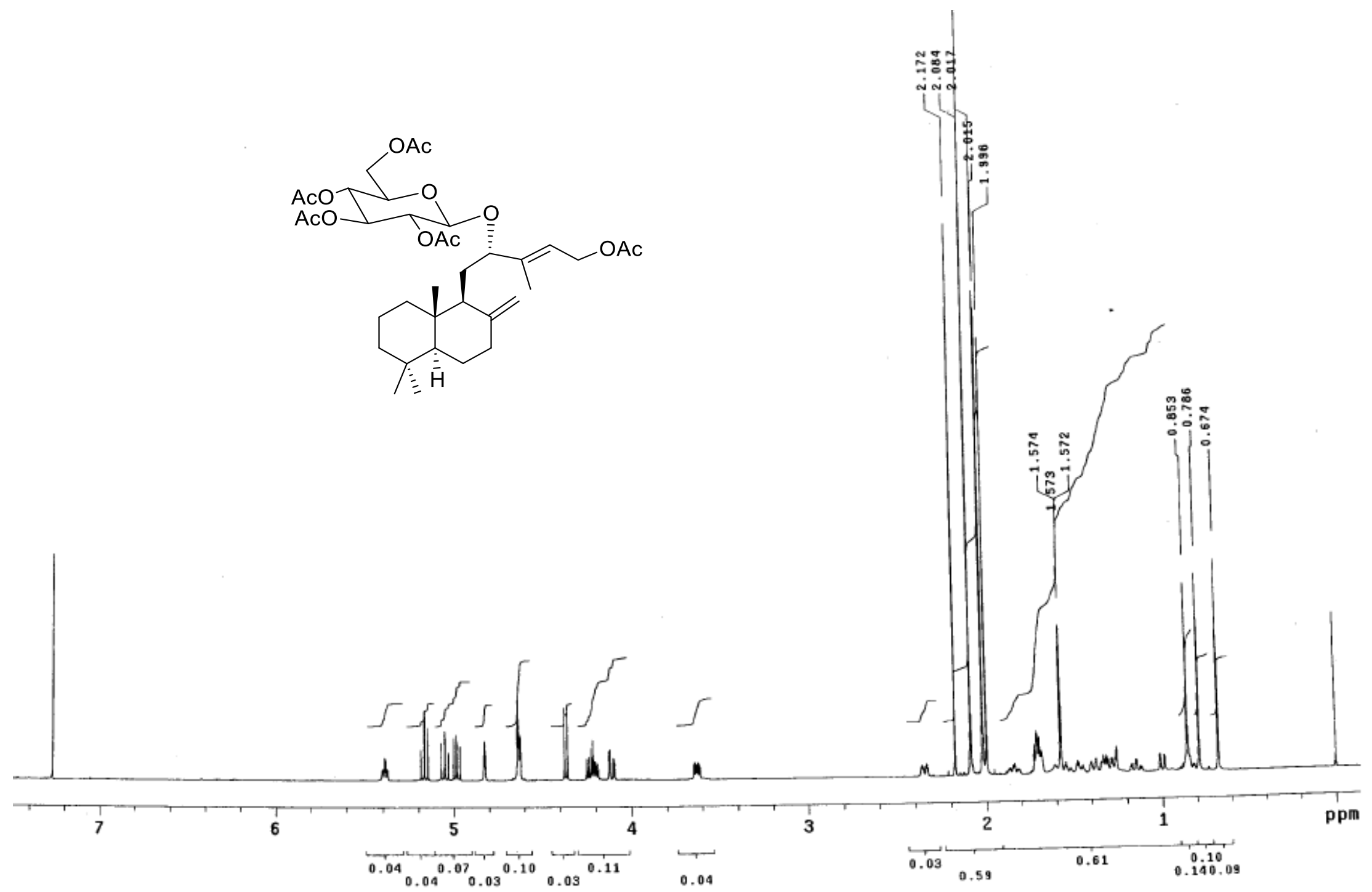

Figure S35. ${ }^{1} \mathrm{H} \mathrm{NMR}$ spectrum of compound $\mathbf{5 a}\left(500 \mathrm{MHz}, \mathrm{CDCl}_{3}\right)$. 


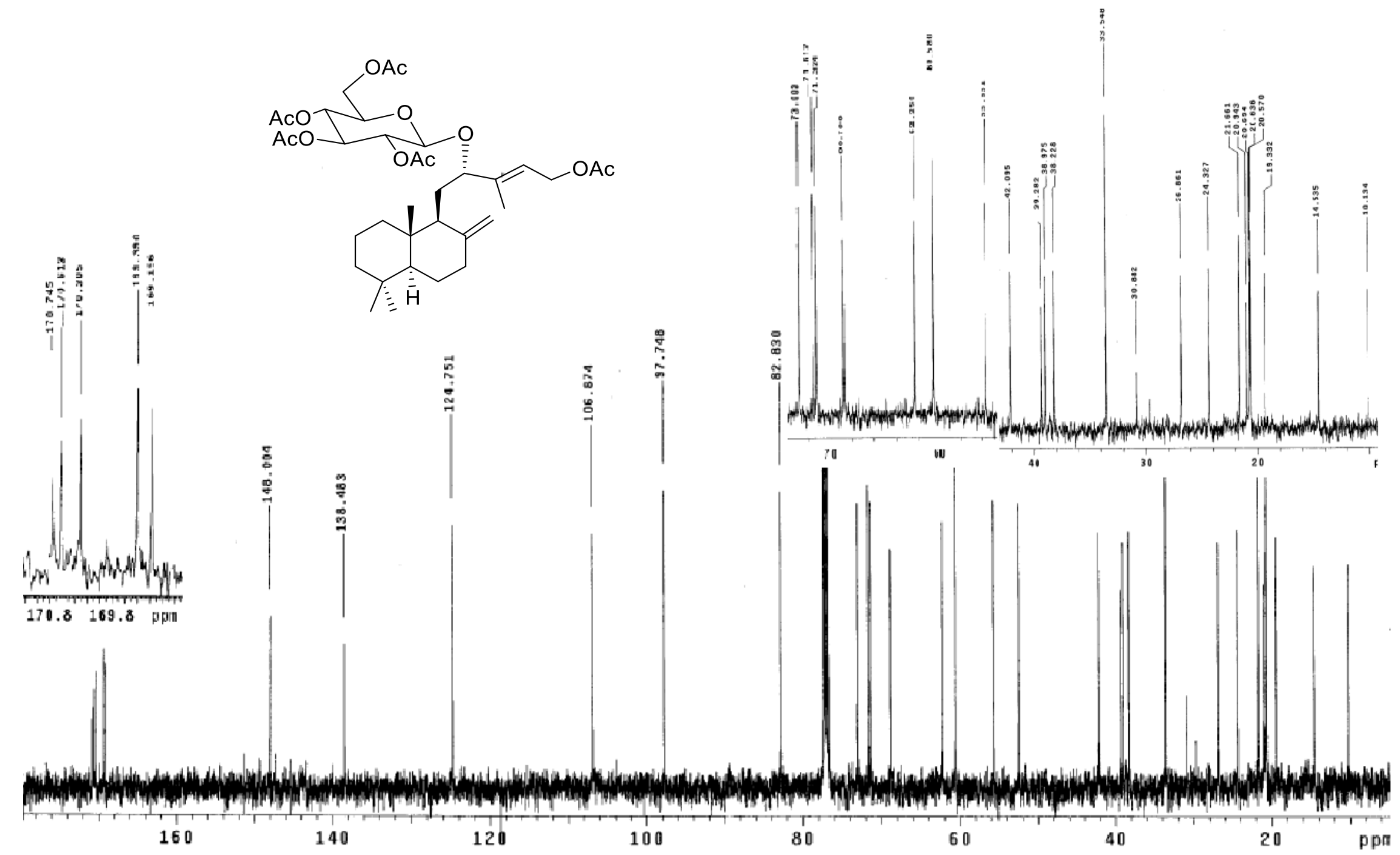

Figure S36. ${ }^{13} \mathrm{C}$ NMR spectrum of compound $5 \mathbf{a}\left(125 \mathrm{MHz}, \mathrm{CDCl}_{3}\right)$. 

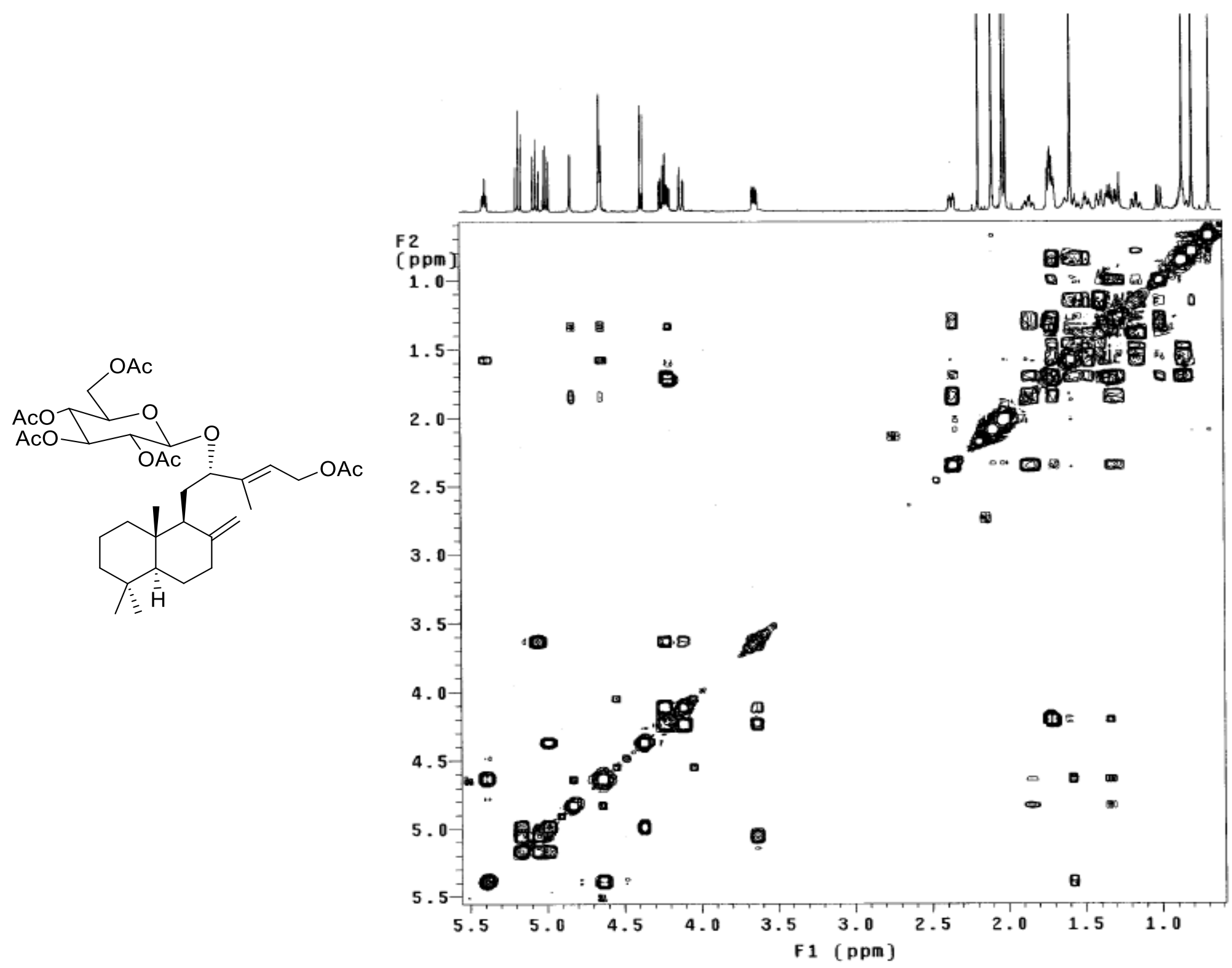

Figure S37. COSY spectrum of compound 5a $\left(\mathrm{CDCl}_{3}\right)$. 


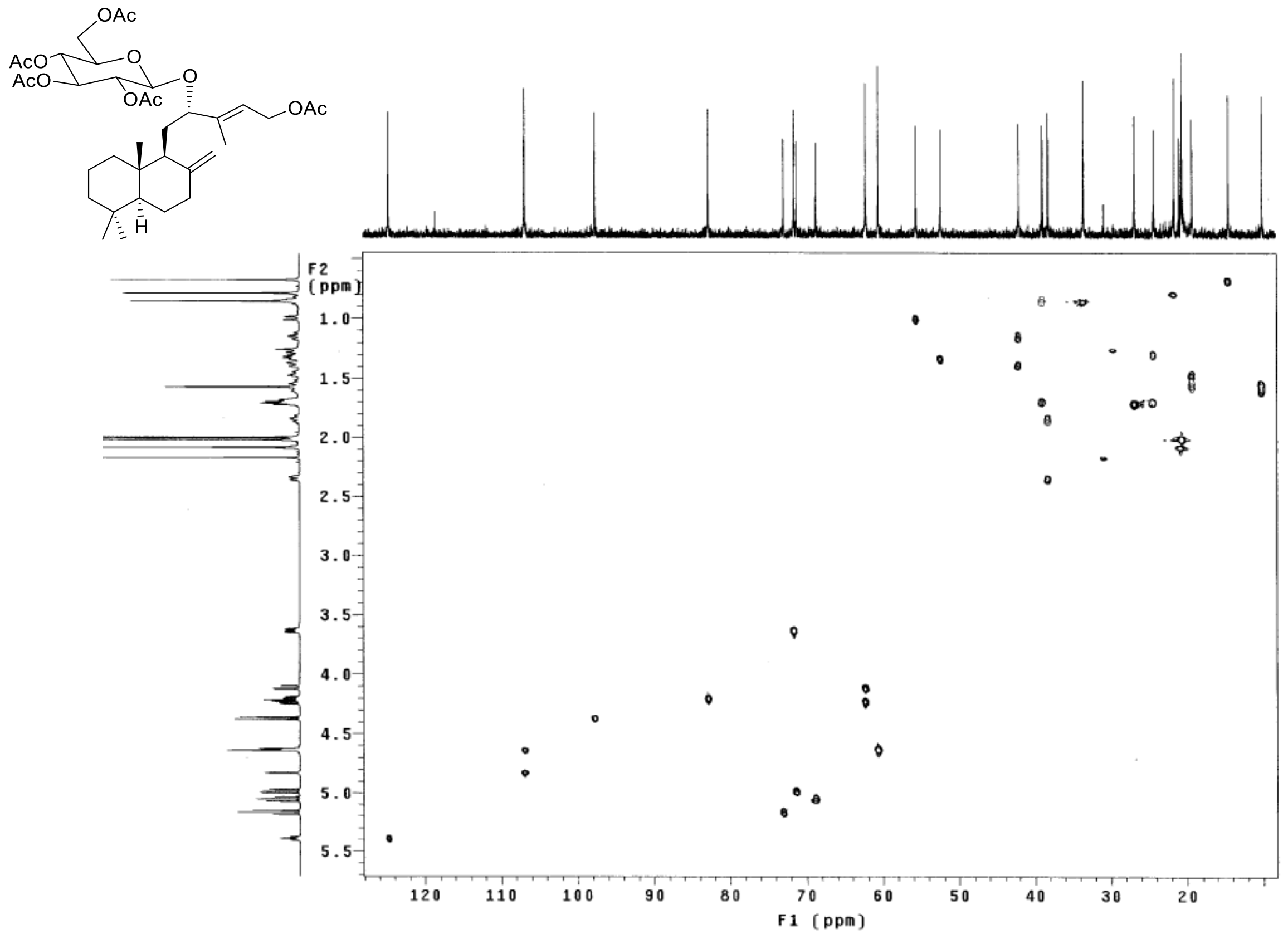

Figure S38. HSQC spectrum of compound $\mathbf{5 a}\left(\mathrm{CDCl}_{3}\right)$. 


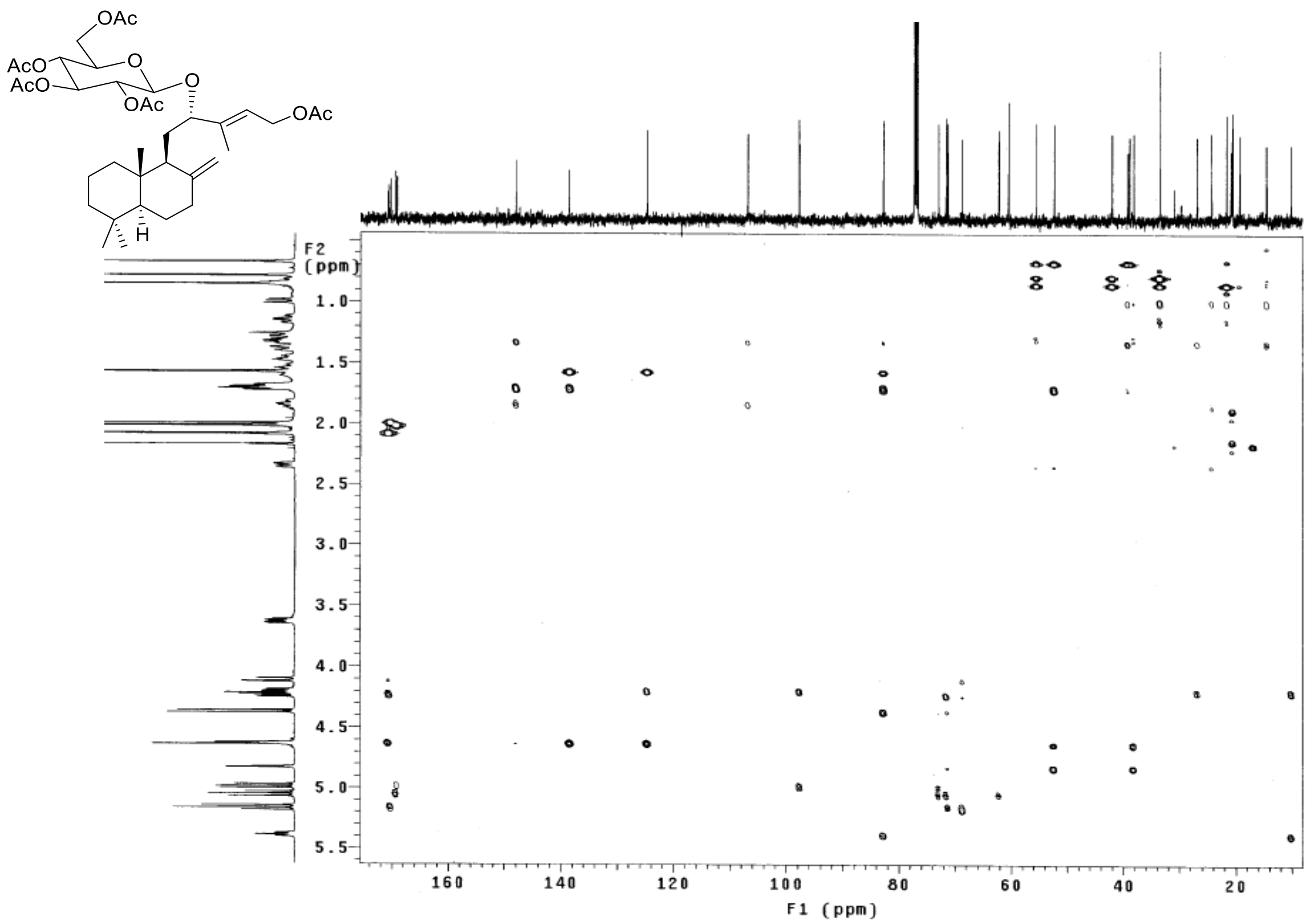

Figure S39. HMBC spectrum of compound 5a $\left(\mathrm{CDCl}_{3}\right)$. 

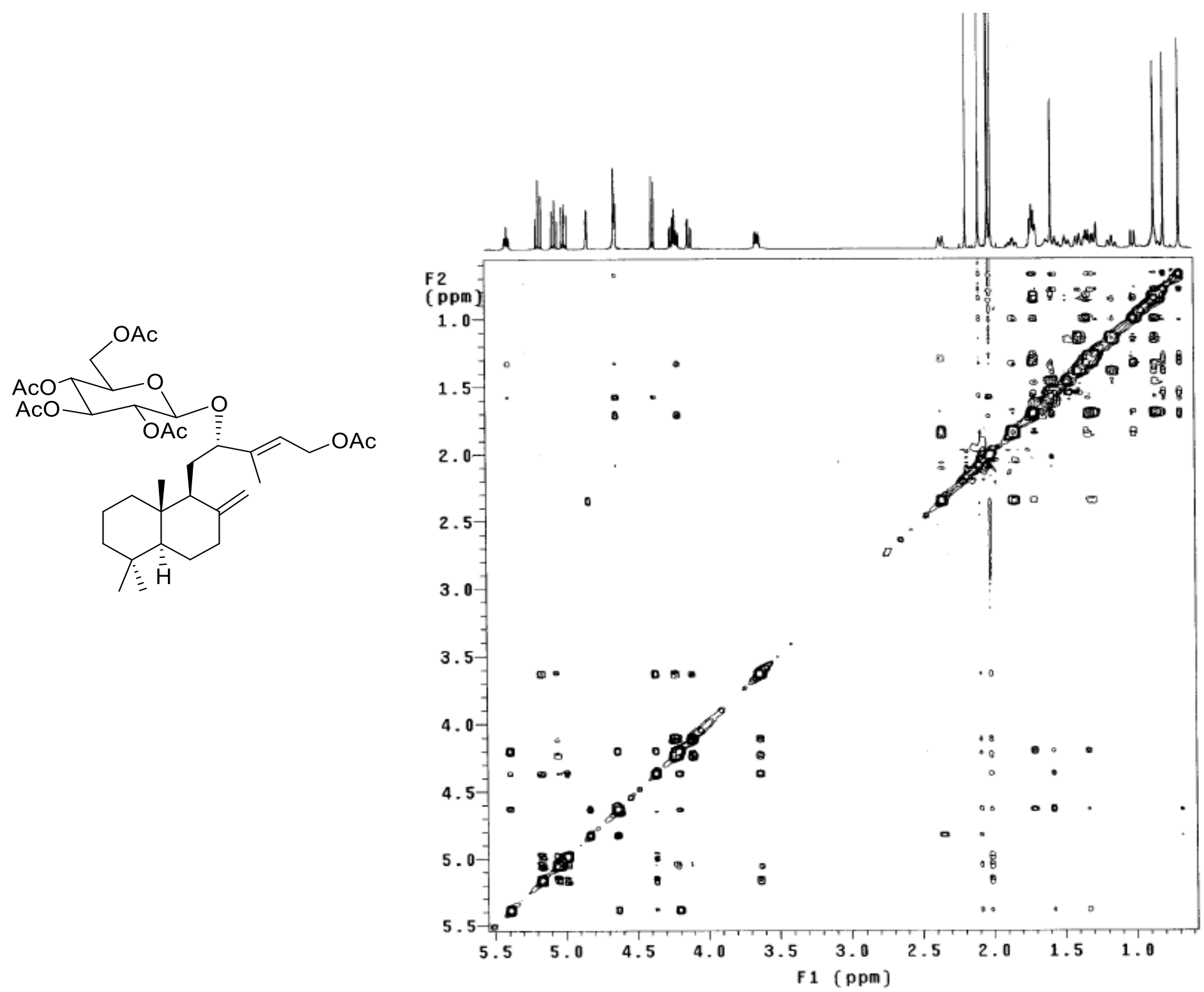

Figure S40. NOESY spectrum of compound $\mathbf{5 a}\left(\mathrm{CDCl}_{3}\right)$. 


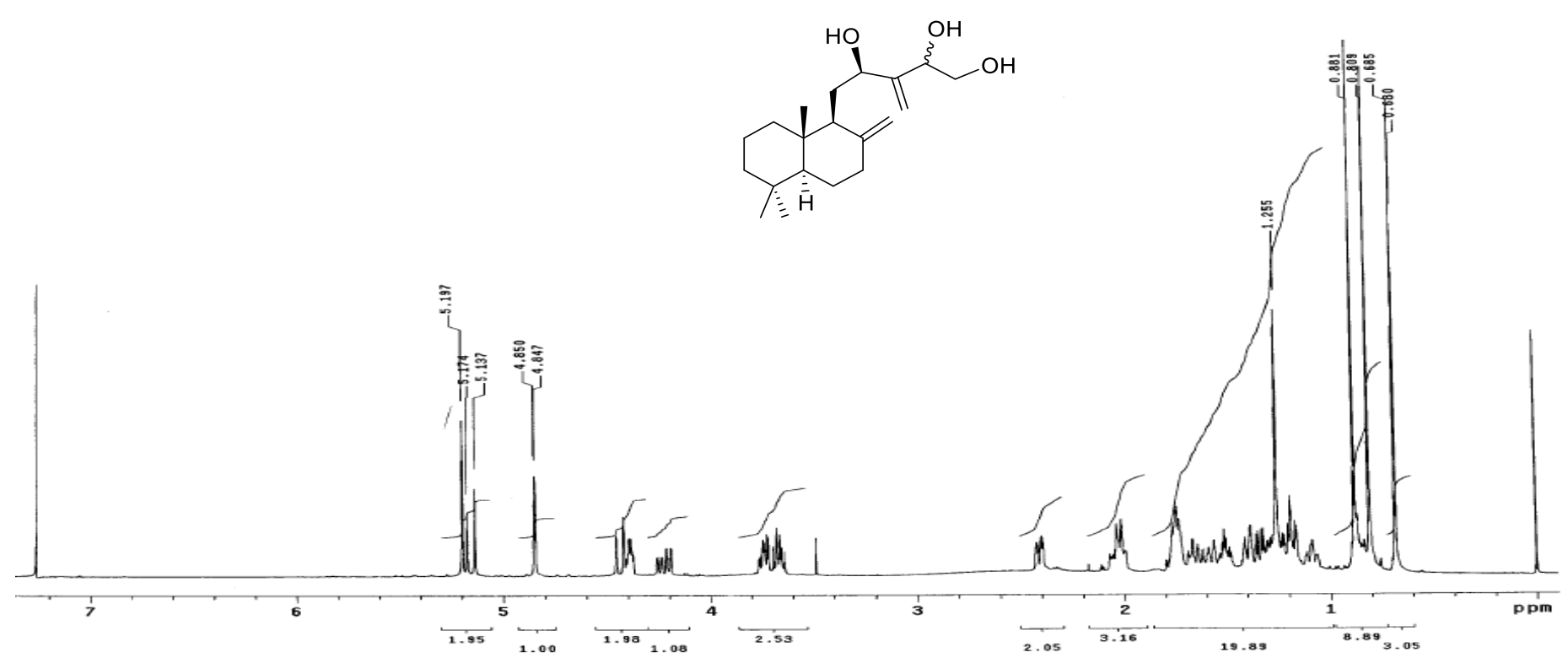

B

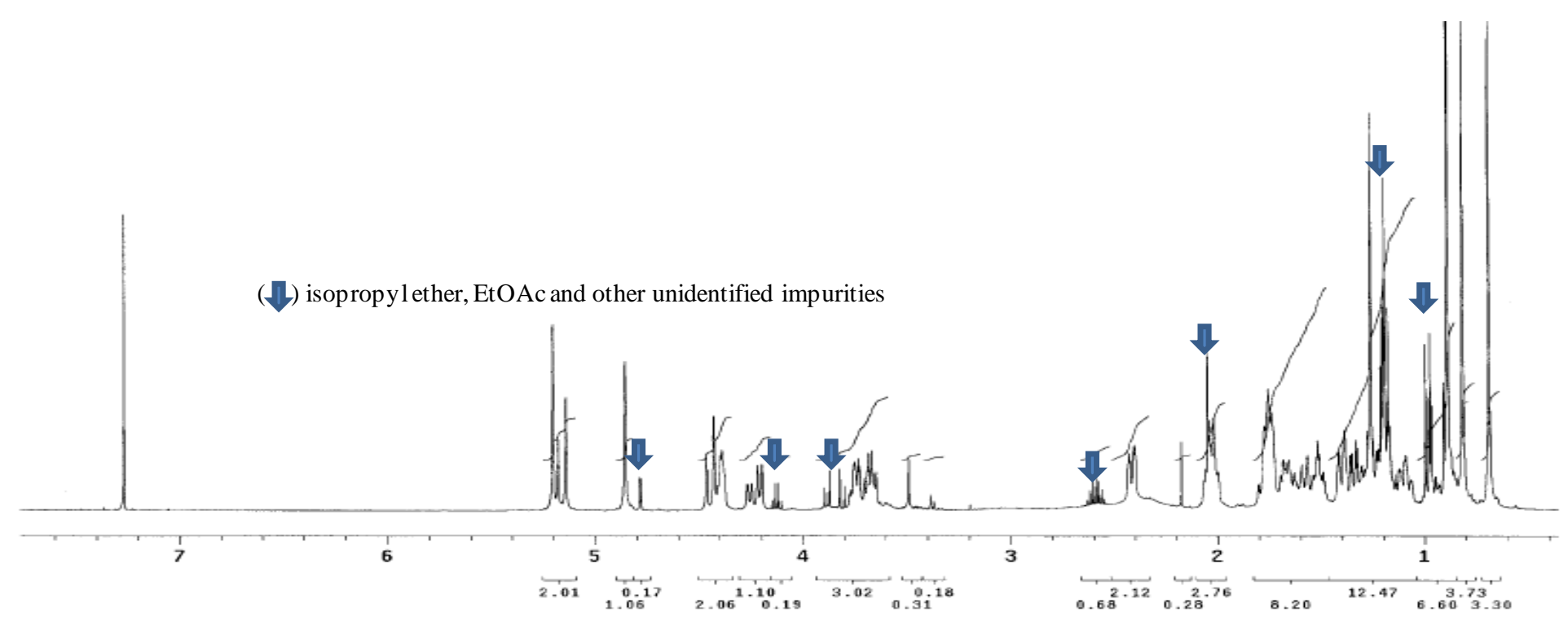

Figure S41. ${ }^{1} \mathrm{H}$ NMR spectra of natural (A) and semisynthetic (B) compounds $6 / 7\left(125 \mathrm{MHz}, \mathrm{CDCl}_{3}\right)$. 


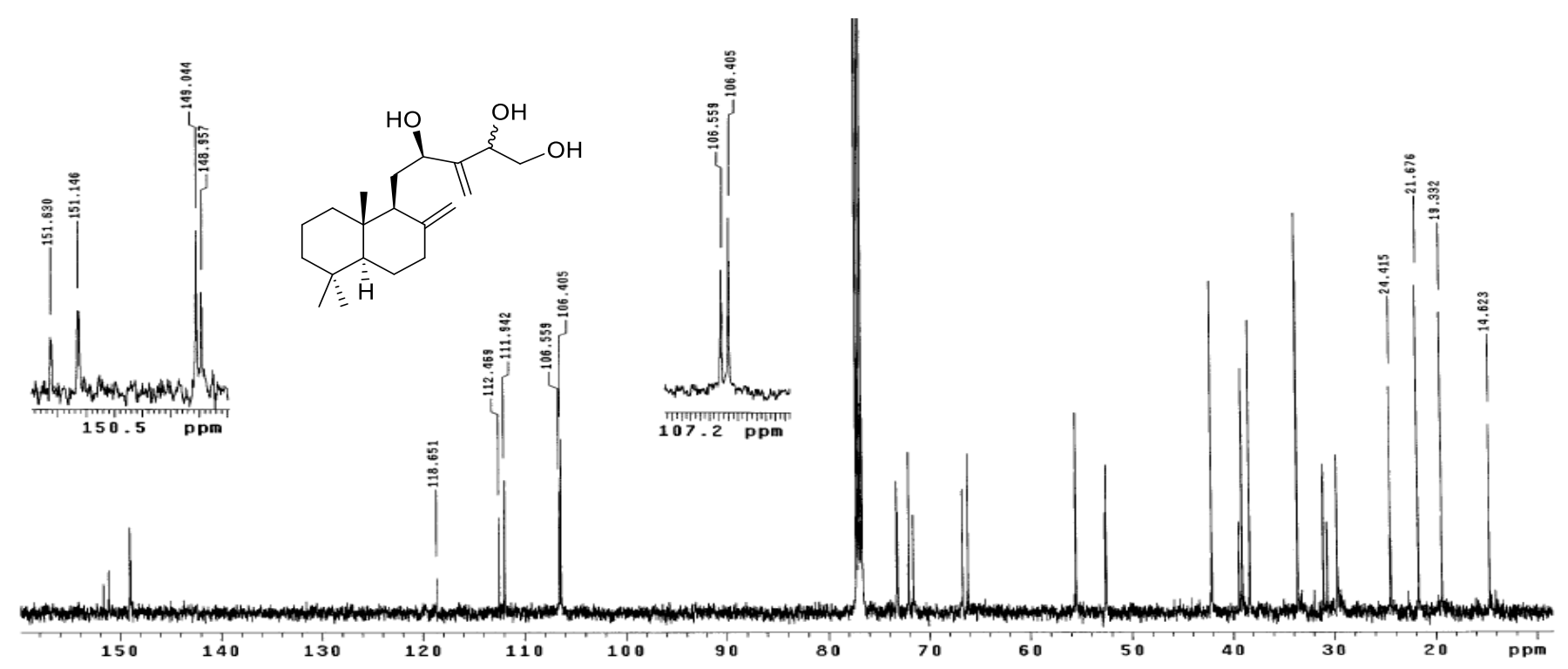

B

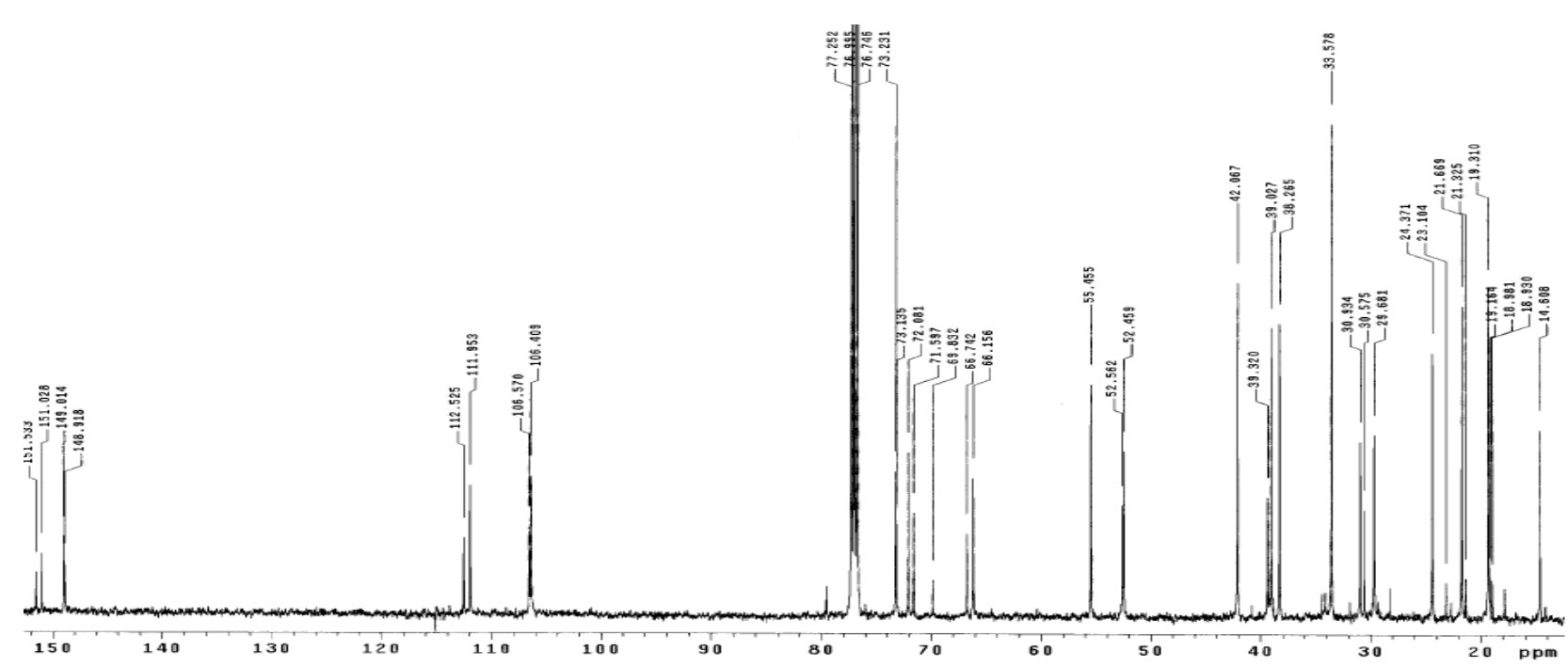

Figure S42. ${ }^{13} \mathrm{C}$ NMR spectra of natural (A) and semisynthetic (B) compounds $6 / 7\left(125 \mathrm{MHz}, \mathrm{CDCl}_{3}\right)$. 


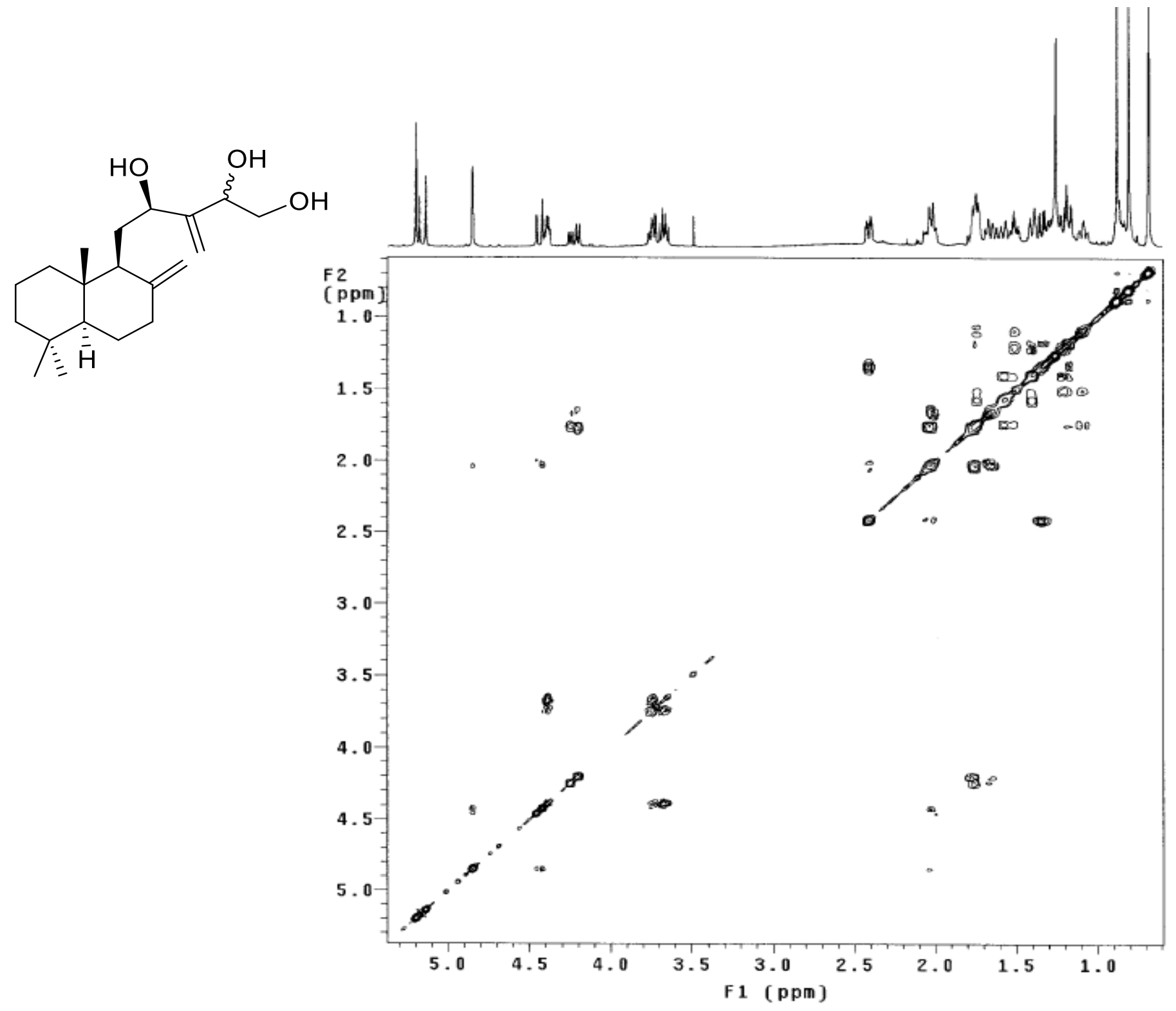

Figure S43. COSY spectrum of natural compounds $6 / 7\left(\mathrm{CDCl}_{3}\right)$. 


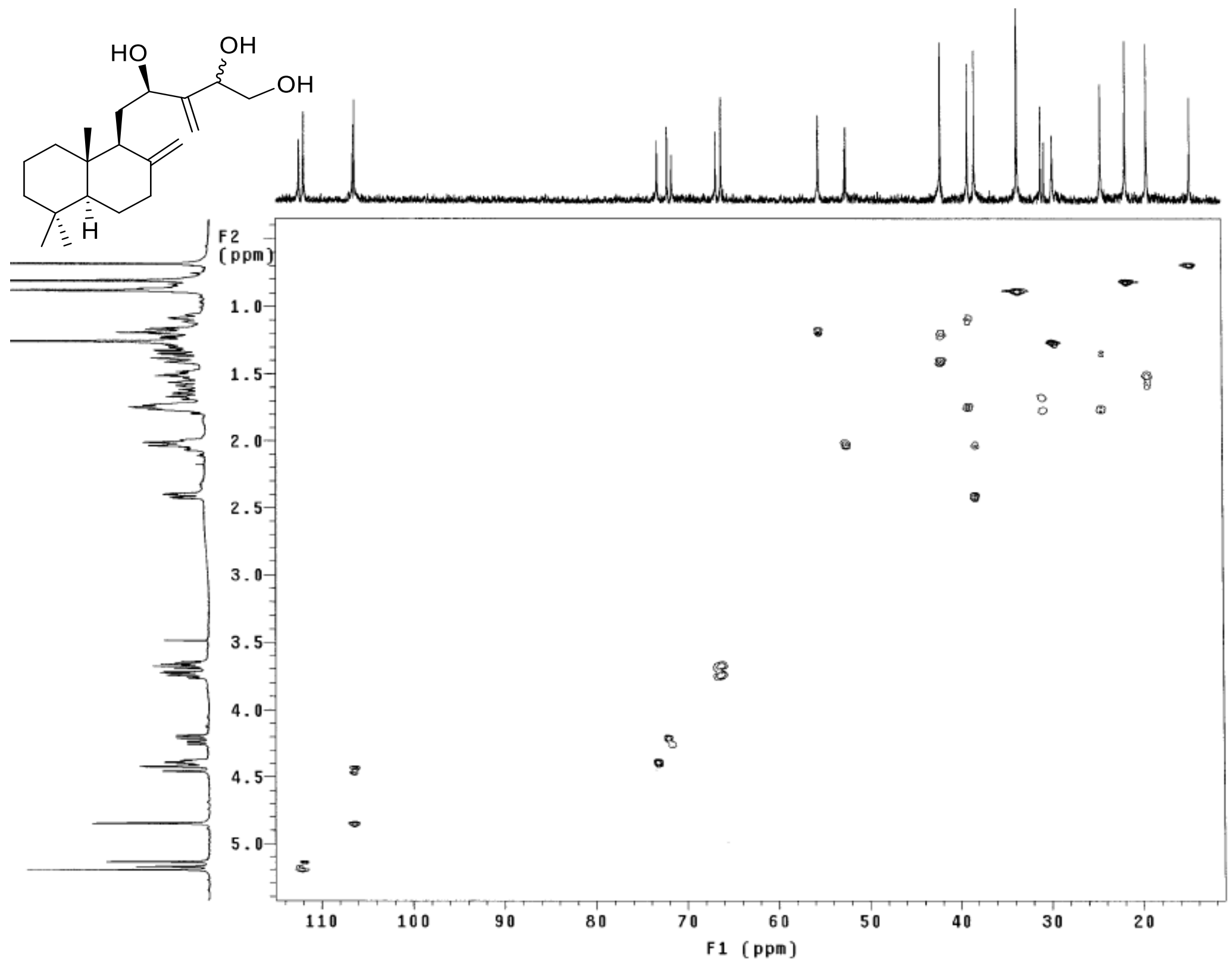

Figure S44. HSQC spectrum of natural compounds $6 / 7\left(\mathrm{CDCl}_{3}\right)$. 


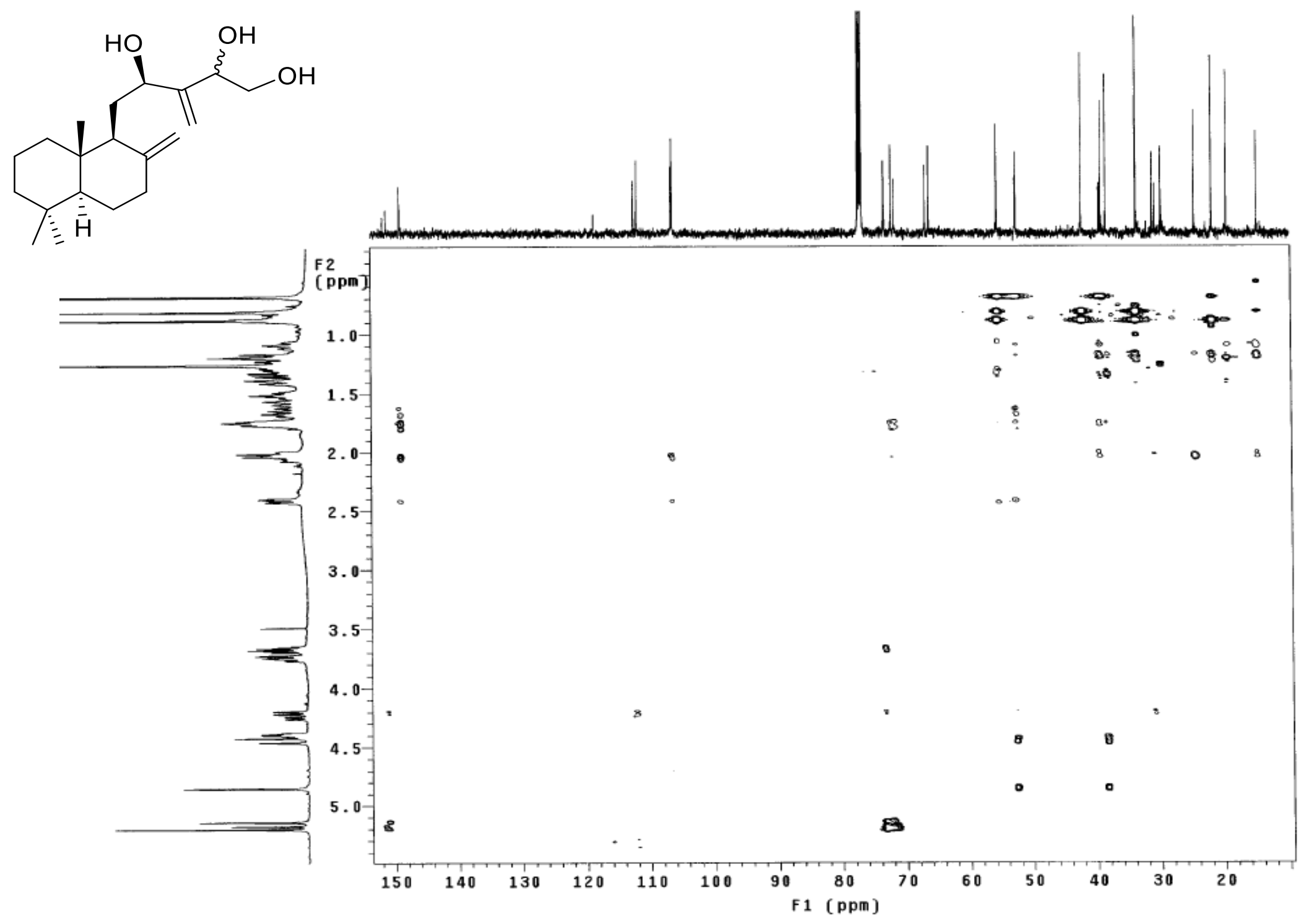

Figure S45. HMBC spectrum of natural compounds $6 / 7\left(\mathrm{CDCl}_{3}\right)$. 


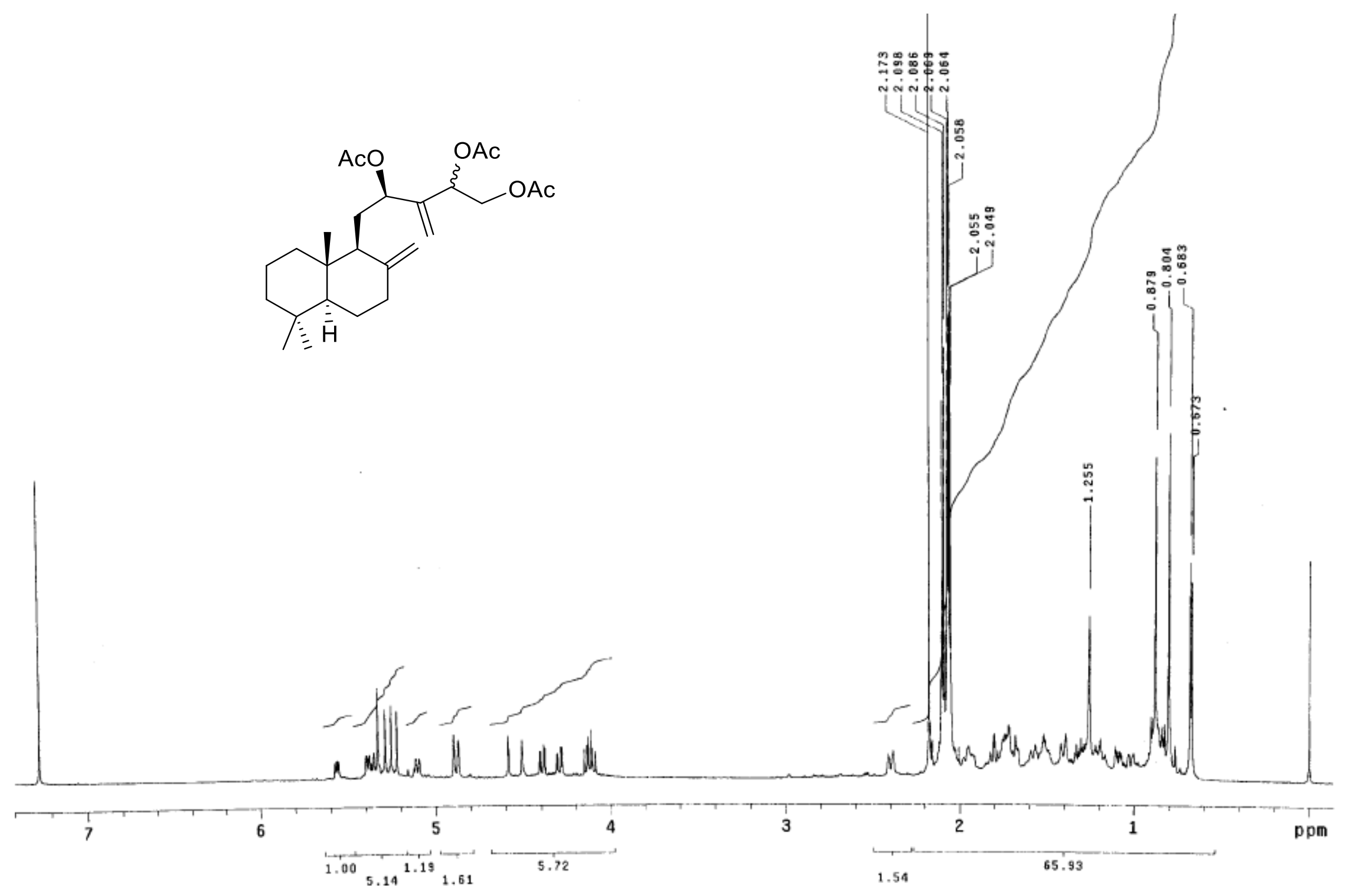

Figure S46. ${ }^{1} \mathrm{H}$ NMR spectrum of compounds $6 \mathbf{6} / 7 \mathbf{a}\left(500 \mathrm{MHz}, \mathrm{CDCl}_{3}\right)$. 


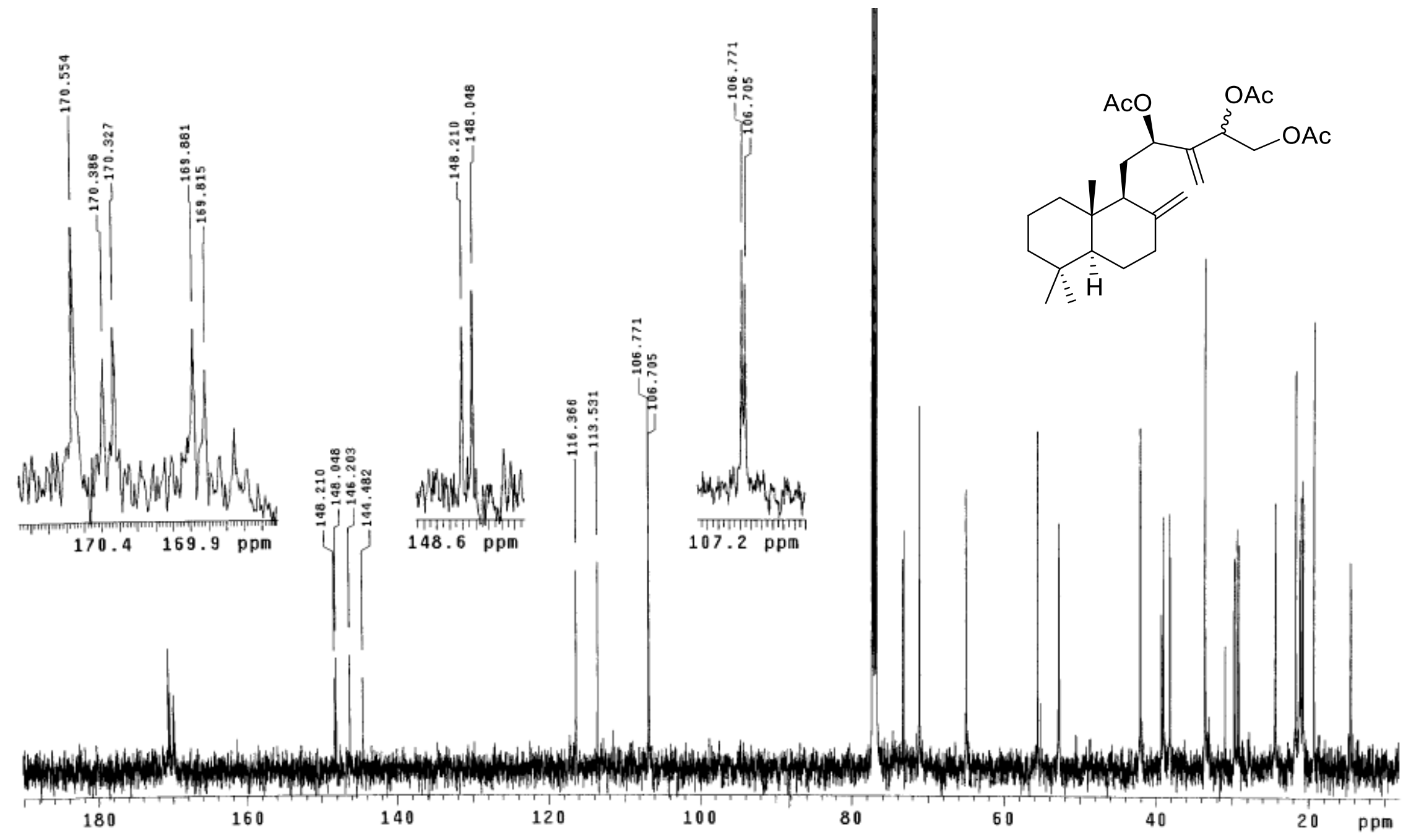

Figure S47. ${ }^{13} \mathrm{C}$ NMR spectrum of compounds $\mathbf{6 a} / 7 \mathbf{a}\left(125 \mathrm{MHz}, \mathrm{CDCl}_{3}\right)$. 
A

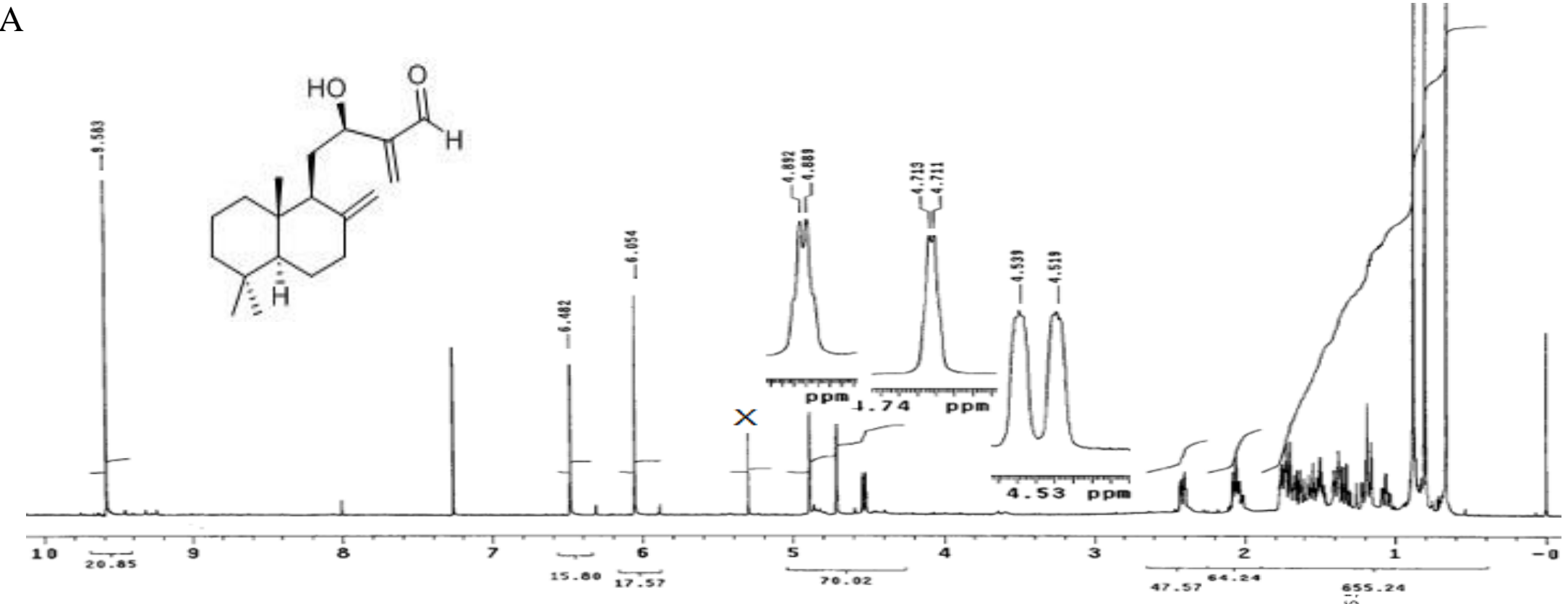

$\mathrm{B}$

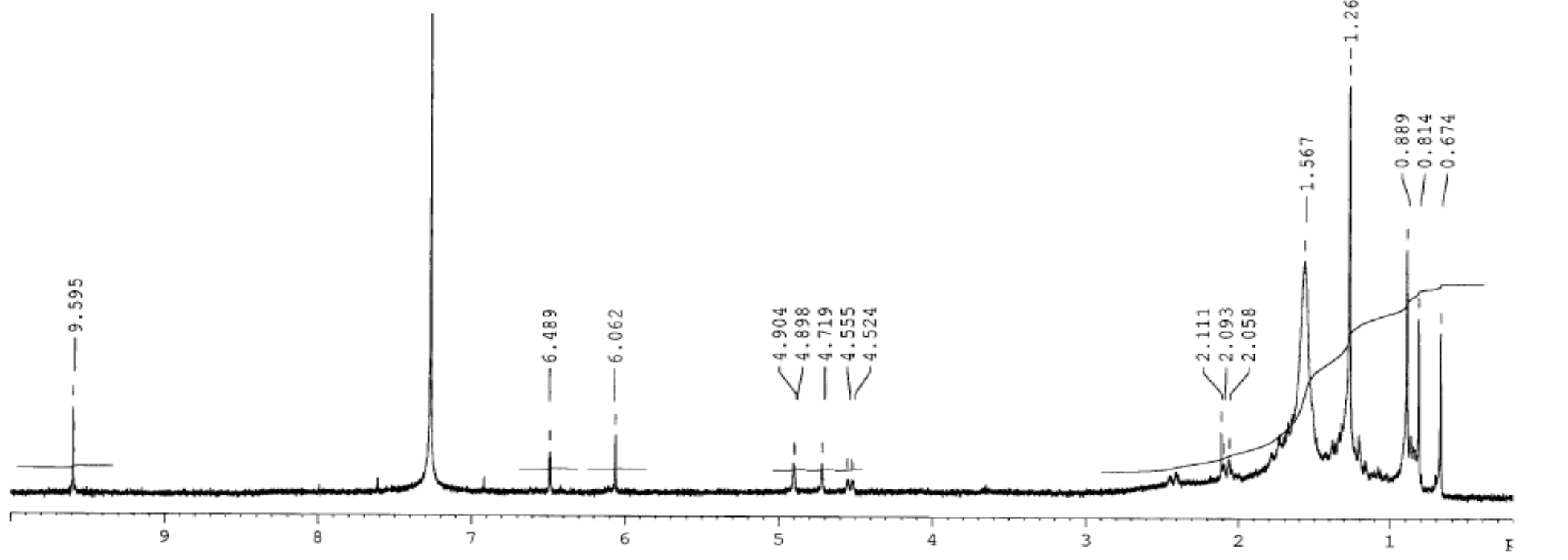

Figure S48. ${ }^{1} \mathrm{H}$ NMR spectra of compound 8 from natural (A) and semisynthetic $\mathbf{6 / 7}$ (B) $\left[500\right.$ (A), 300 (B) MHz, $\left.\mathrm{CDCl}_{3}\right]$. 
<smiles>C=C(C=O)C(O)C[C@H]1C(=C)CC[C@H]2C(C)CCCC12C</smiles>

\section{$\equiv H$}

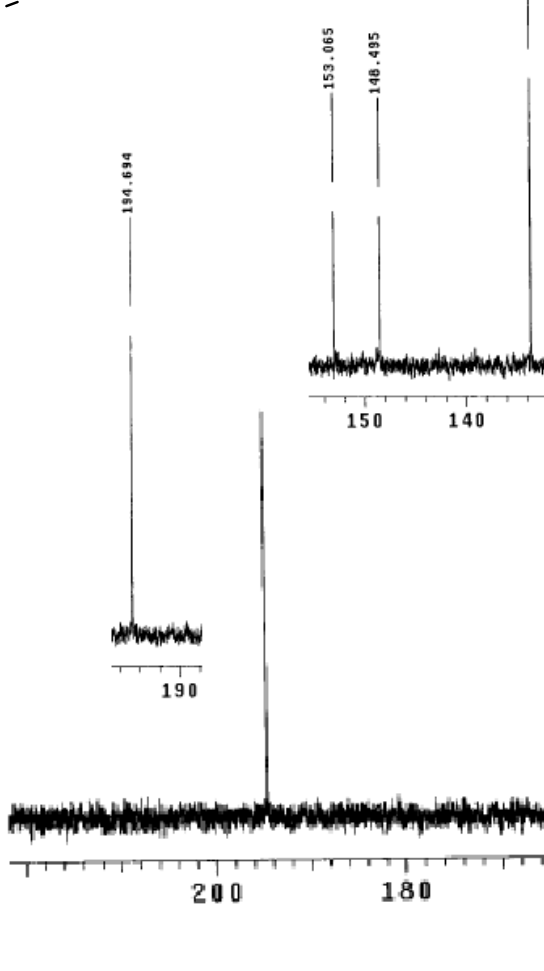

Figure S49. ${ }^{13} \mathrm{C}$ NMR spectrum of compound $8\left(125 \mathrm{MHz}, \mathrm{CDCl}_{3}\right)$. 

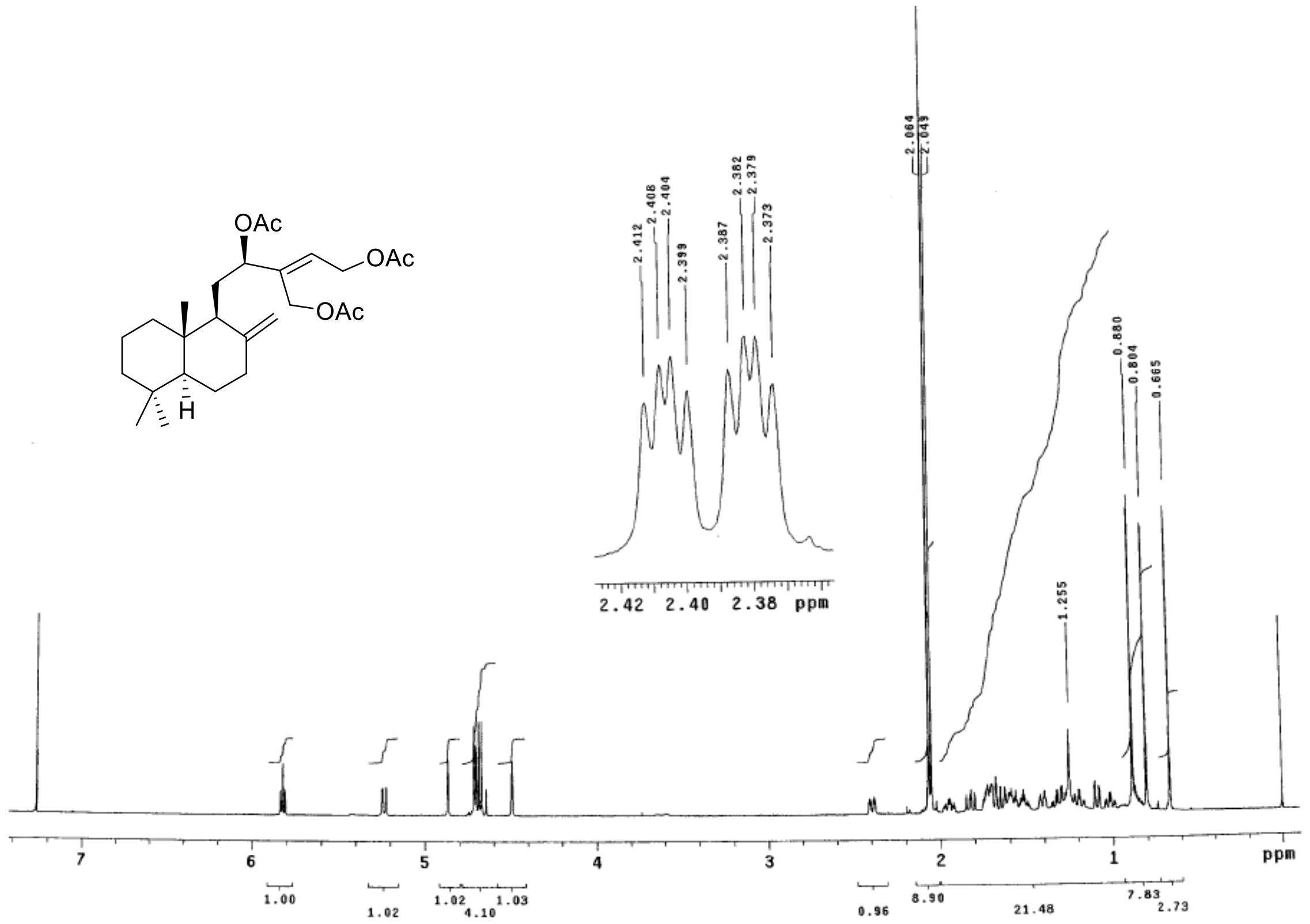

Figure S50. ${ }^{1} \mathrm{H}$ NMR spectrum of compound $9\left(500 \mathrm{MHz}, \mathrm{CDCl}_{3}\right)$. 


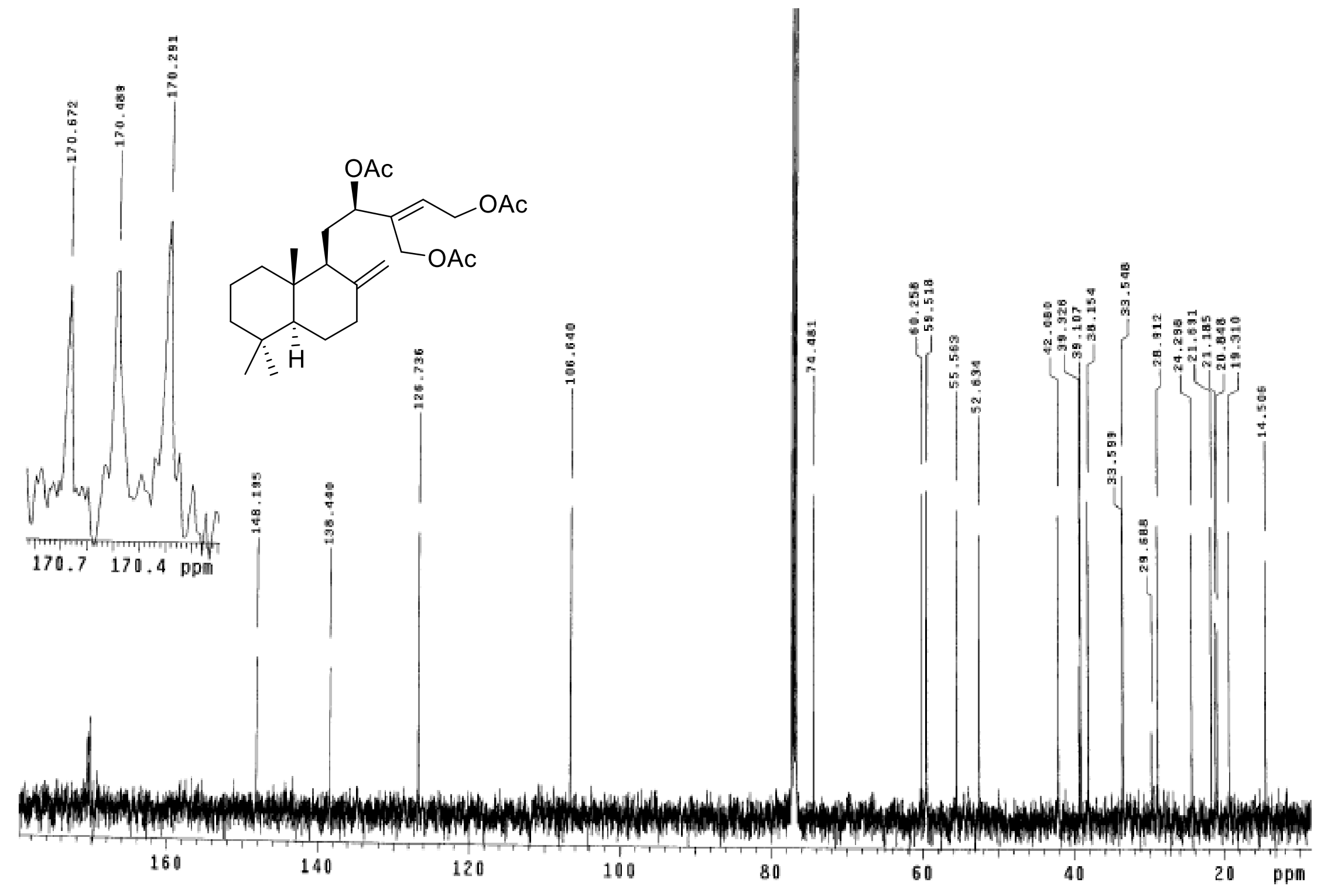

Figure S51. ${ }^{13} \mathrm{C}$ NMR spectrum of compound $9\left(125 \mathrm{MHz}, \mathrm{CDCl}_{3}\right)$. 


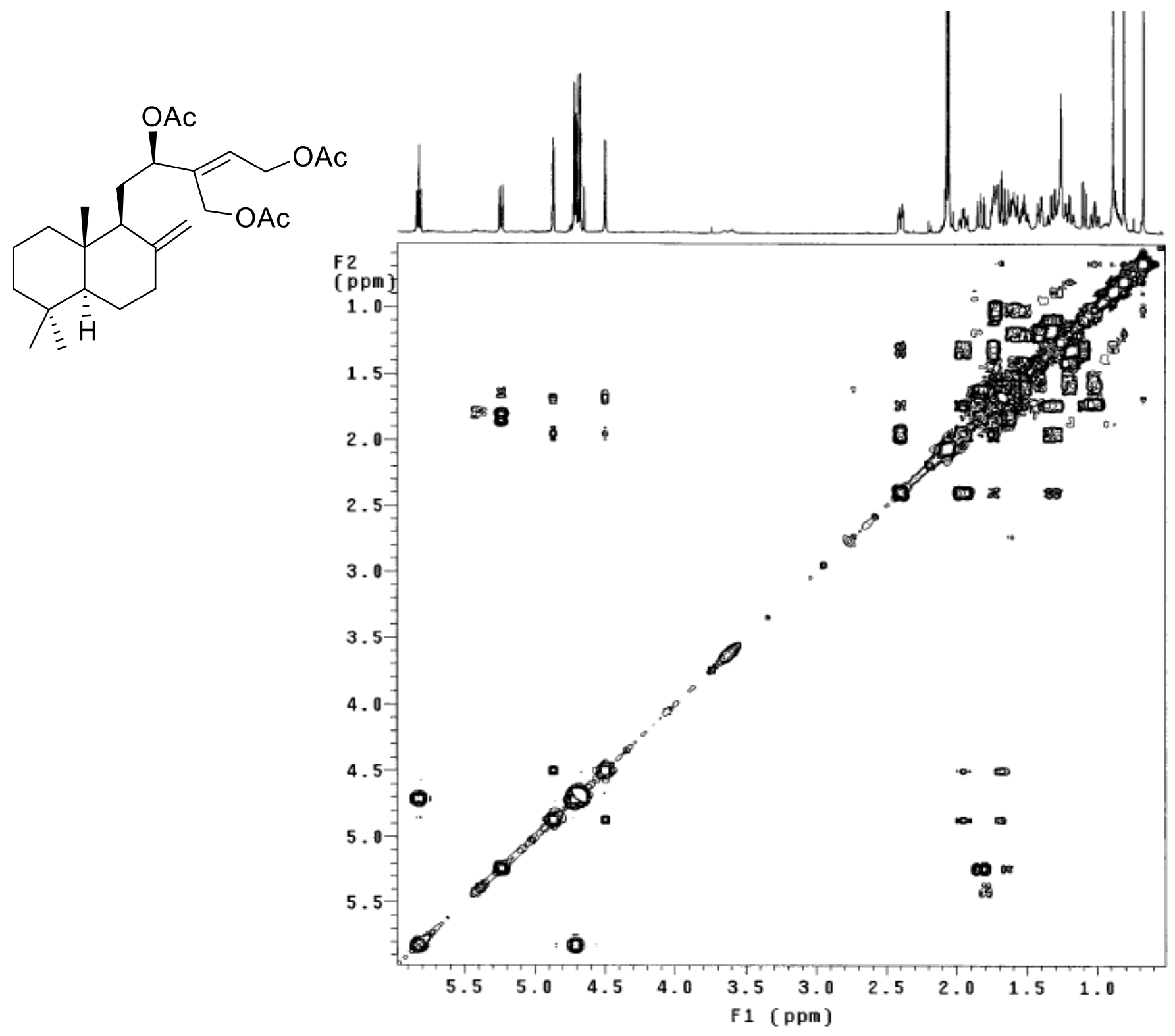

Figure S52. COSY spectrum of compound $9\left(\mathrm{CDCl}_{3}\right)$. 


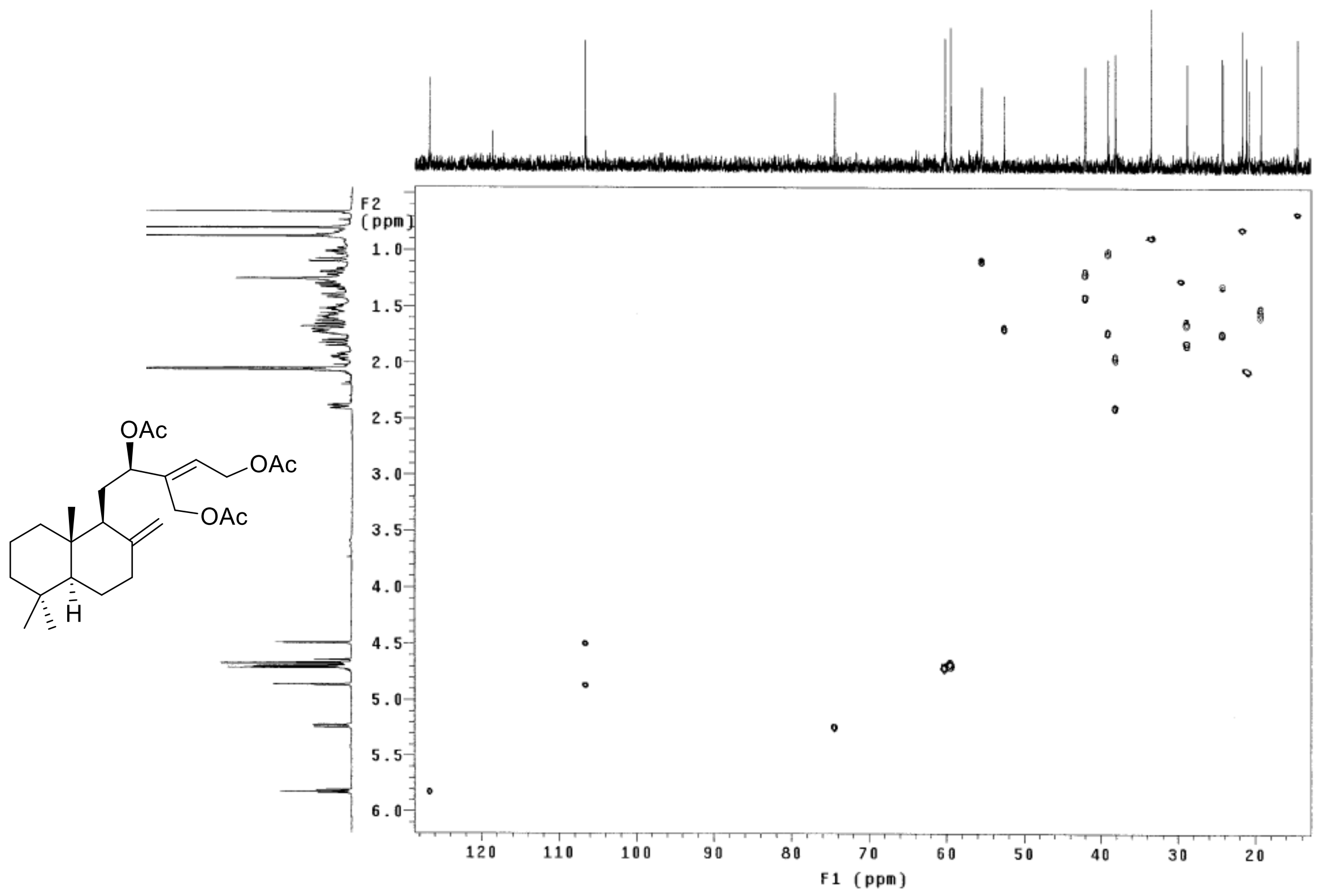

Figure S53. HSQC spectrum of compound $9\left(\mathrm{CDCl}_{3}\right)$. 


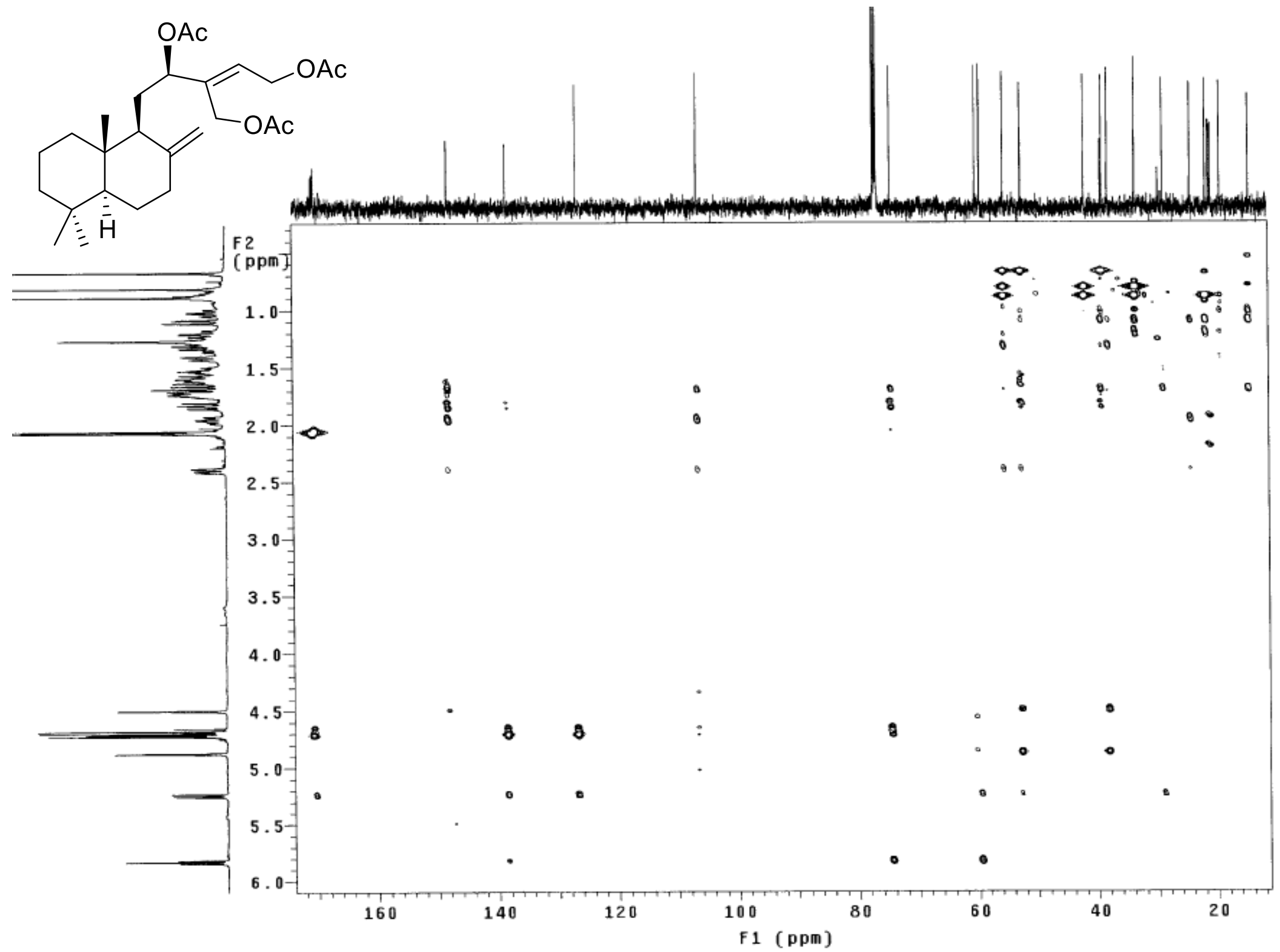

Figure S54. HMBC spectrum of compound $9\left(\mathrm{CDCl}_{3}\right)$. 

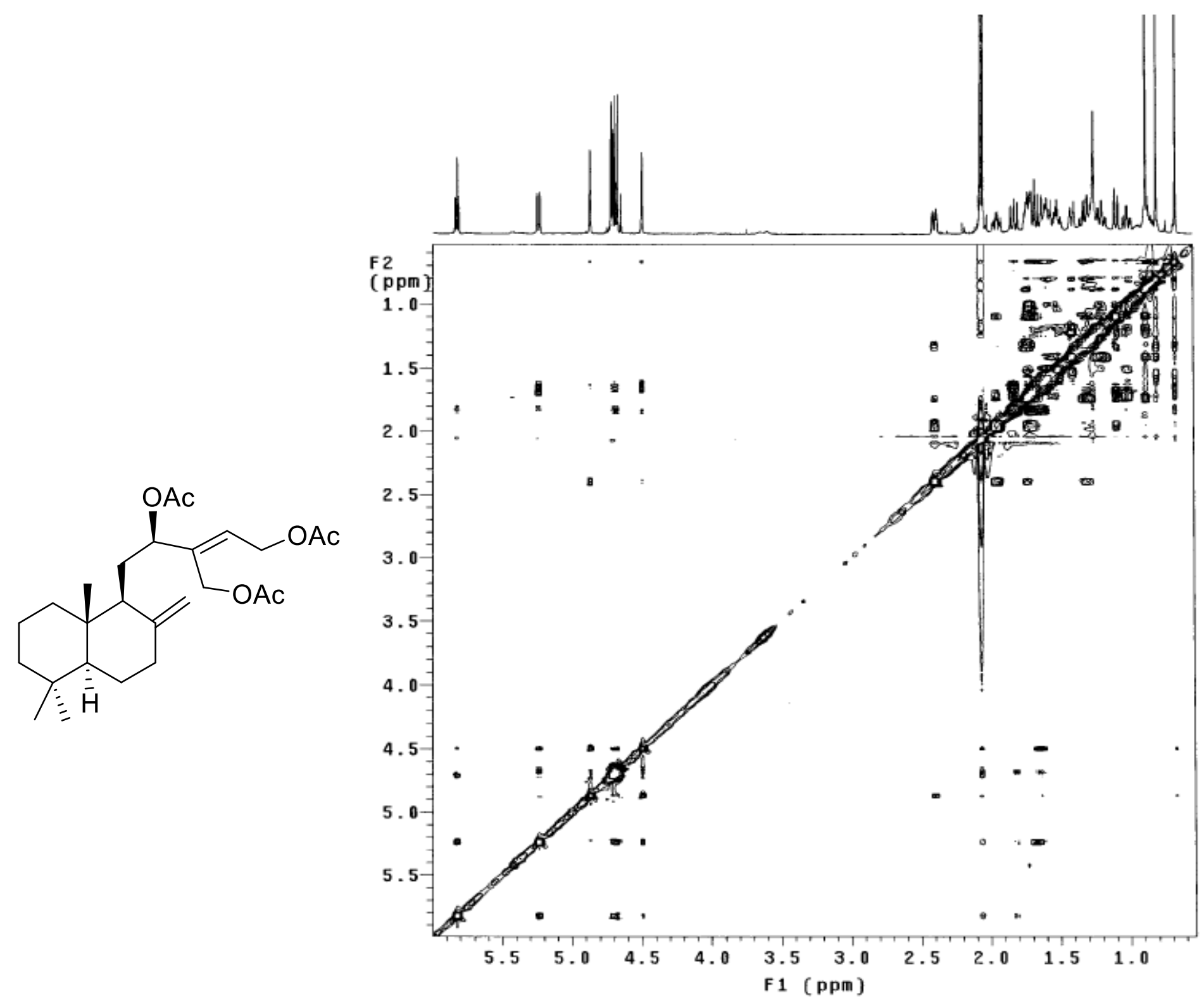

Figure S55. NOESY spectrum of compound $9\left(\mathrm{CDCl}_{3}\right)$. 


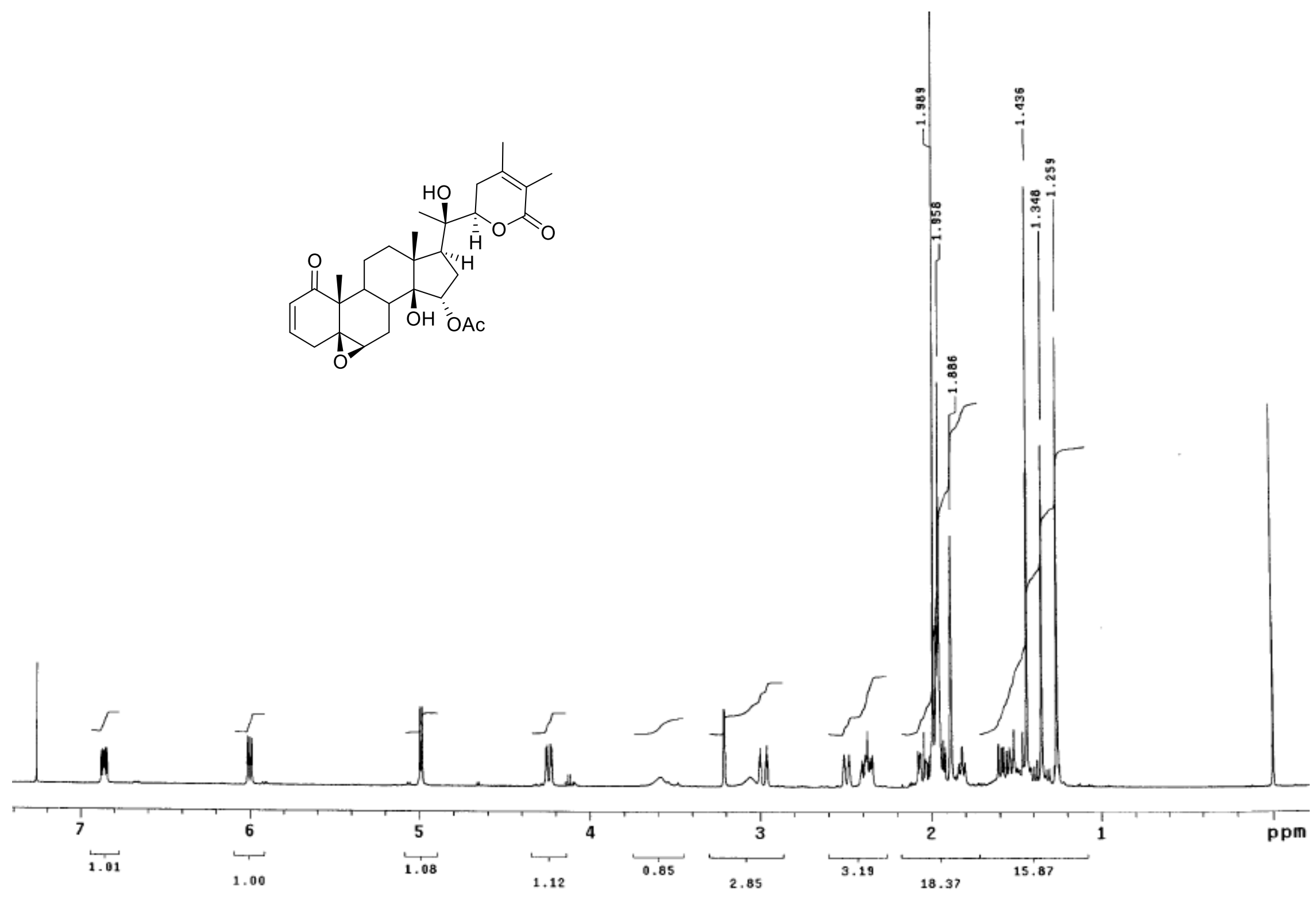

Figure S56. ${ }^{1} \mathrm{H}$ NMR spectrum of compound $14\left(500 \mathrm{MHz}, \mathrm{CDCl}_{3}\right)$. 

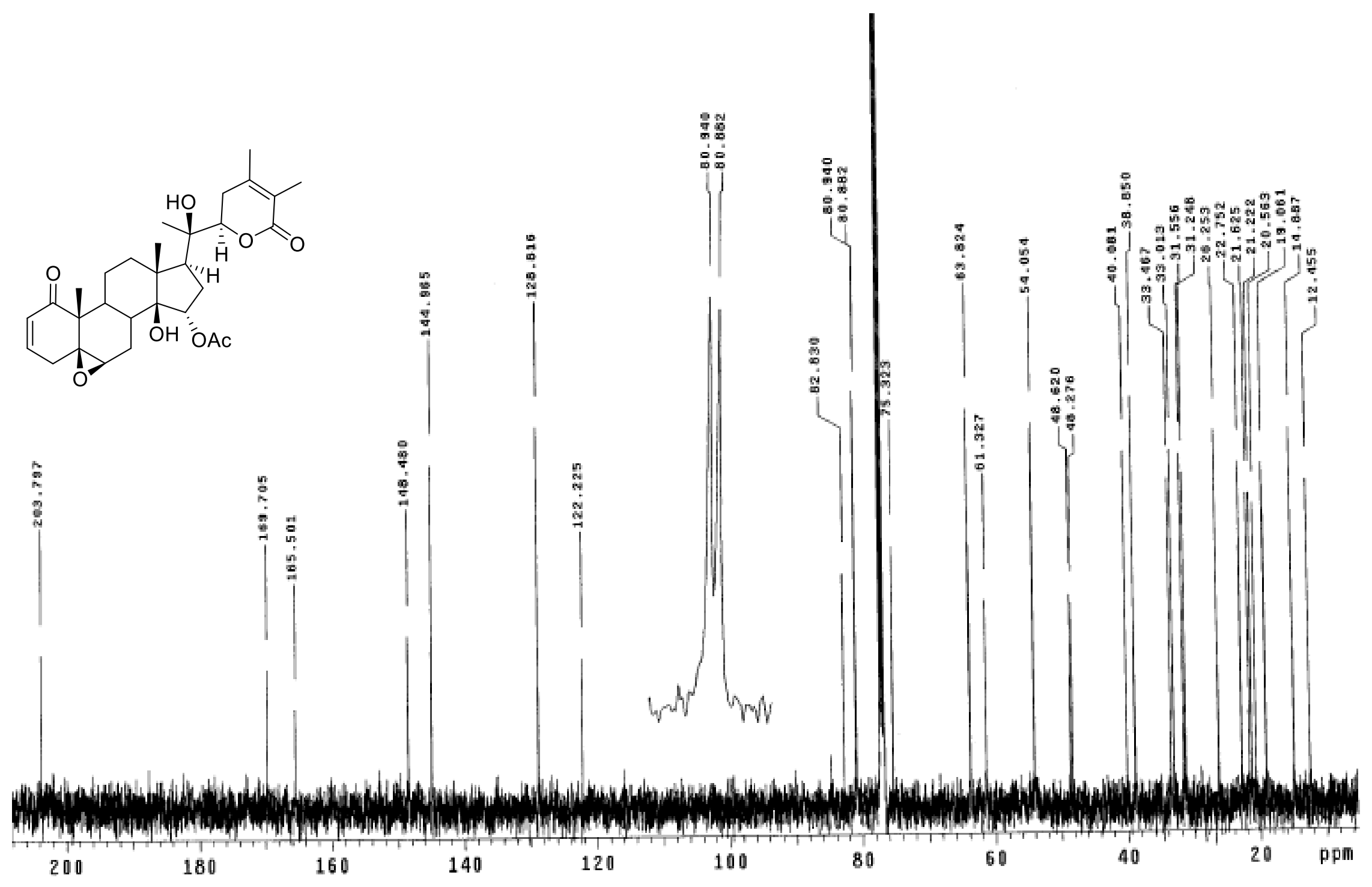

Figure S57. ${ }^{13} \mathrm{C}$ NMR spectrum of compound $14\left(125 \mathrm{MHz}, \mathrm{CDCl}_{3}\right)$. 

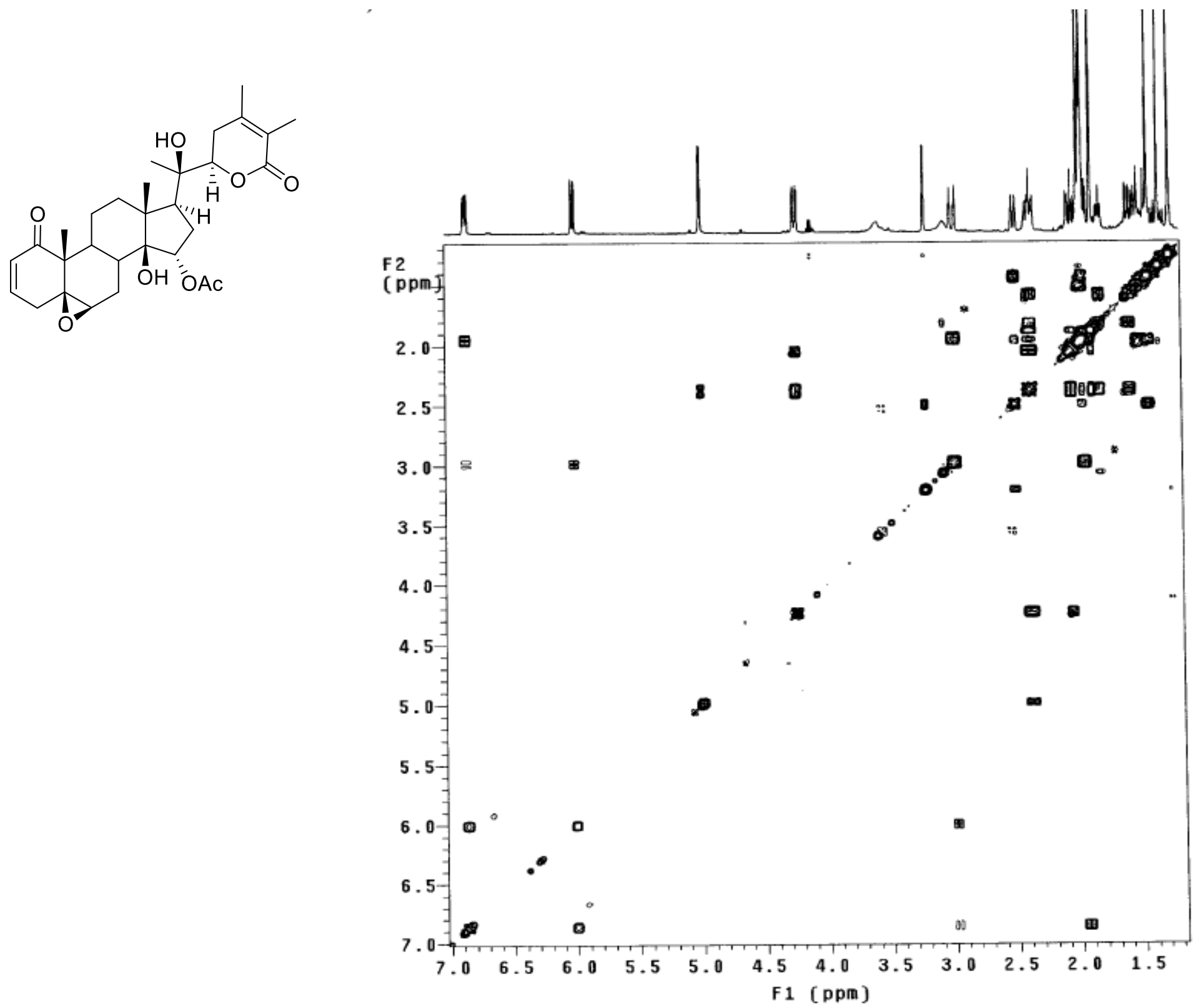

Figure S58. COSY spectrum of compound $14\left(\mathrm{CDCl}_{3}\right)$. 


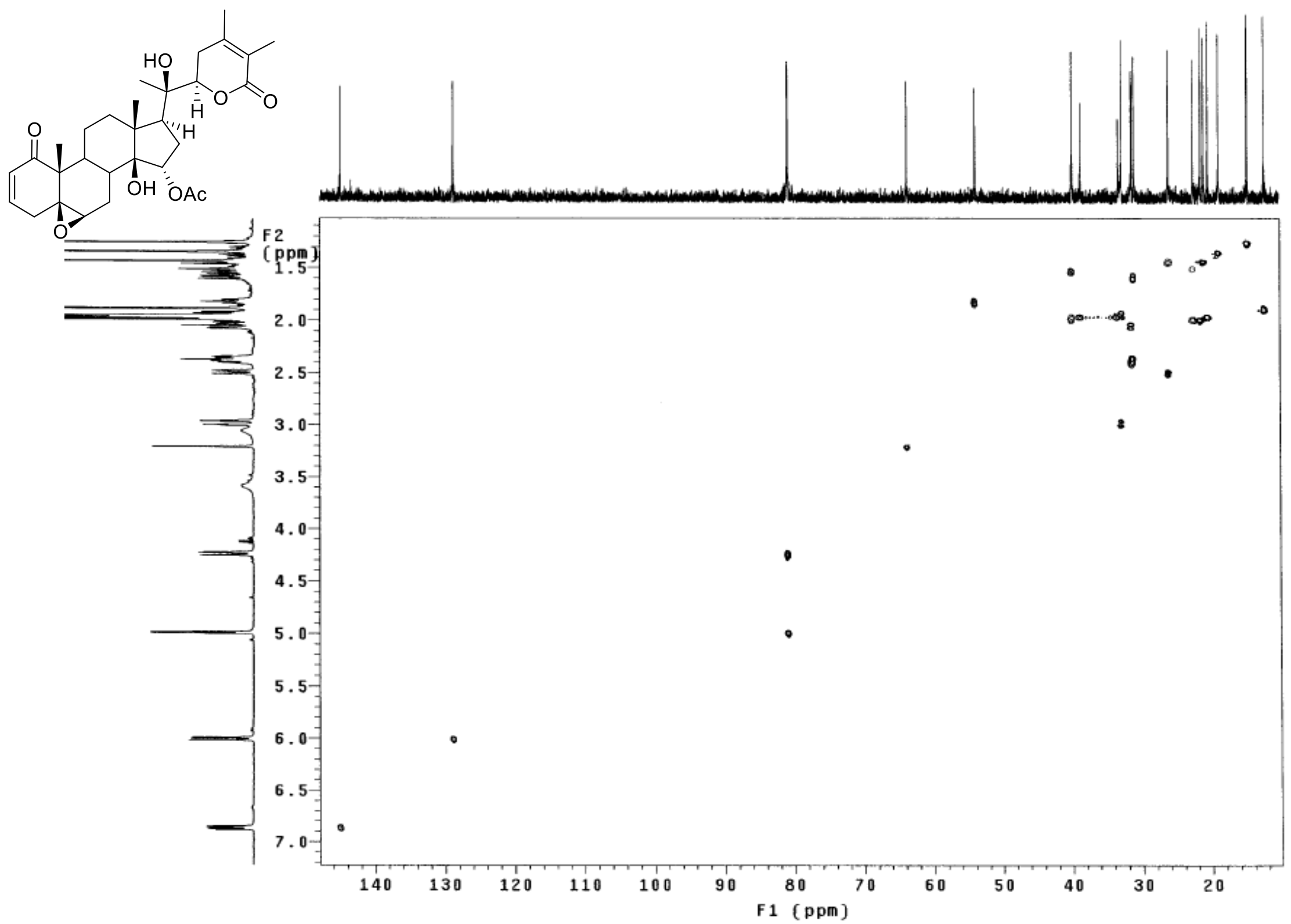

Figure S59. HSQC spectrum of compound $14\left(\mathrm{CDCl}_{3}\right)$. 


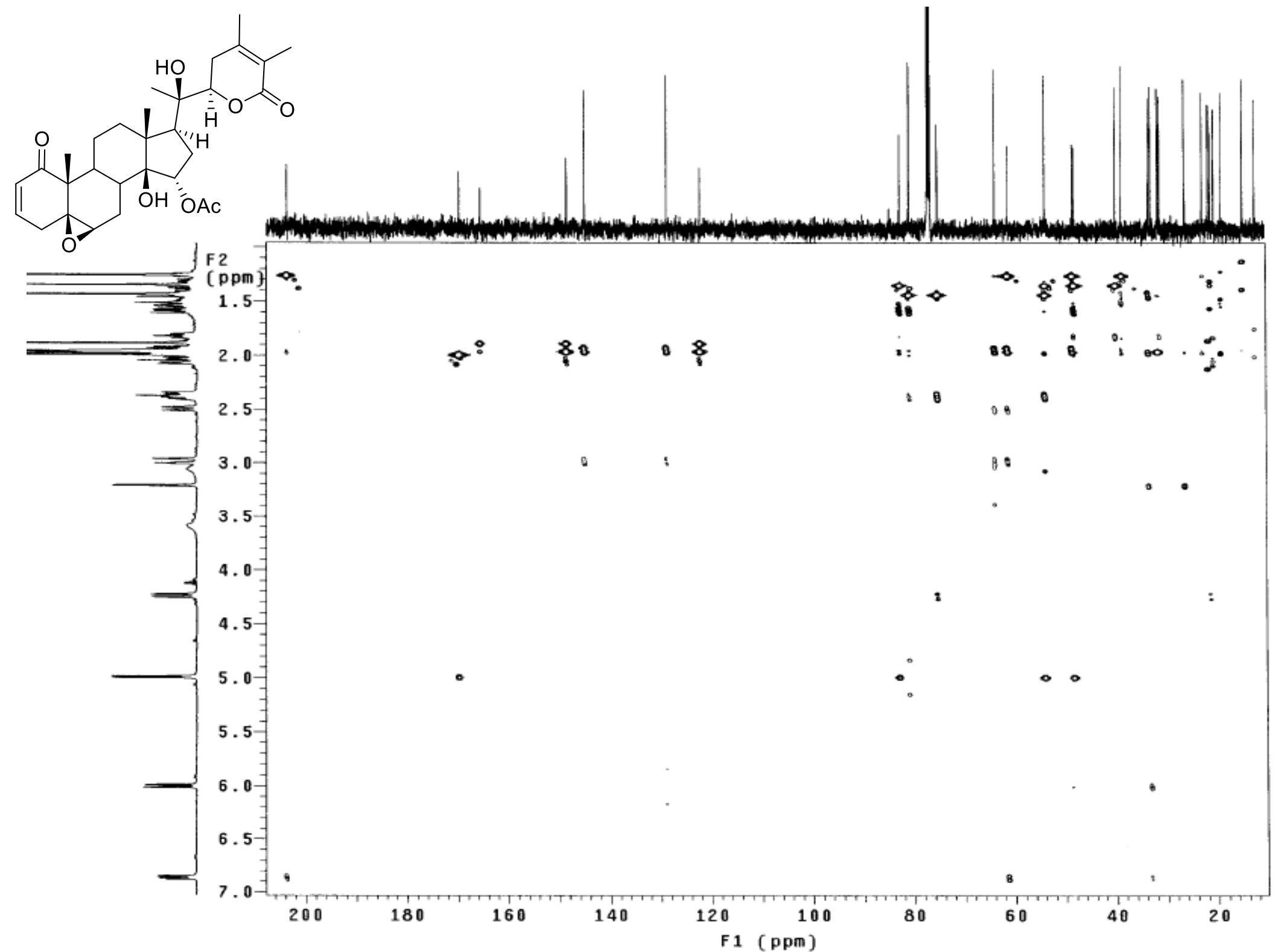

Figure S60. HMBC spectrum of compound $14\left(\mathrm{CDCl}_{3}\right)$. 


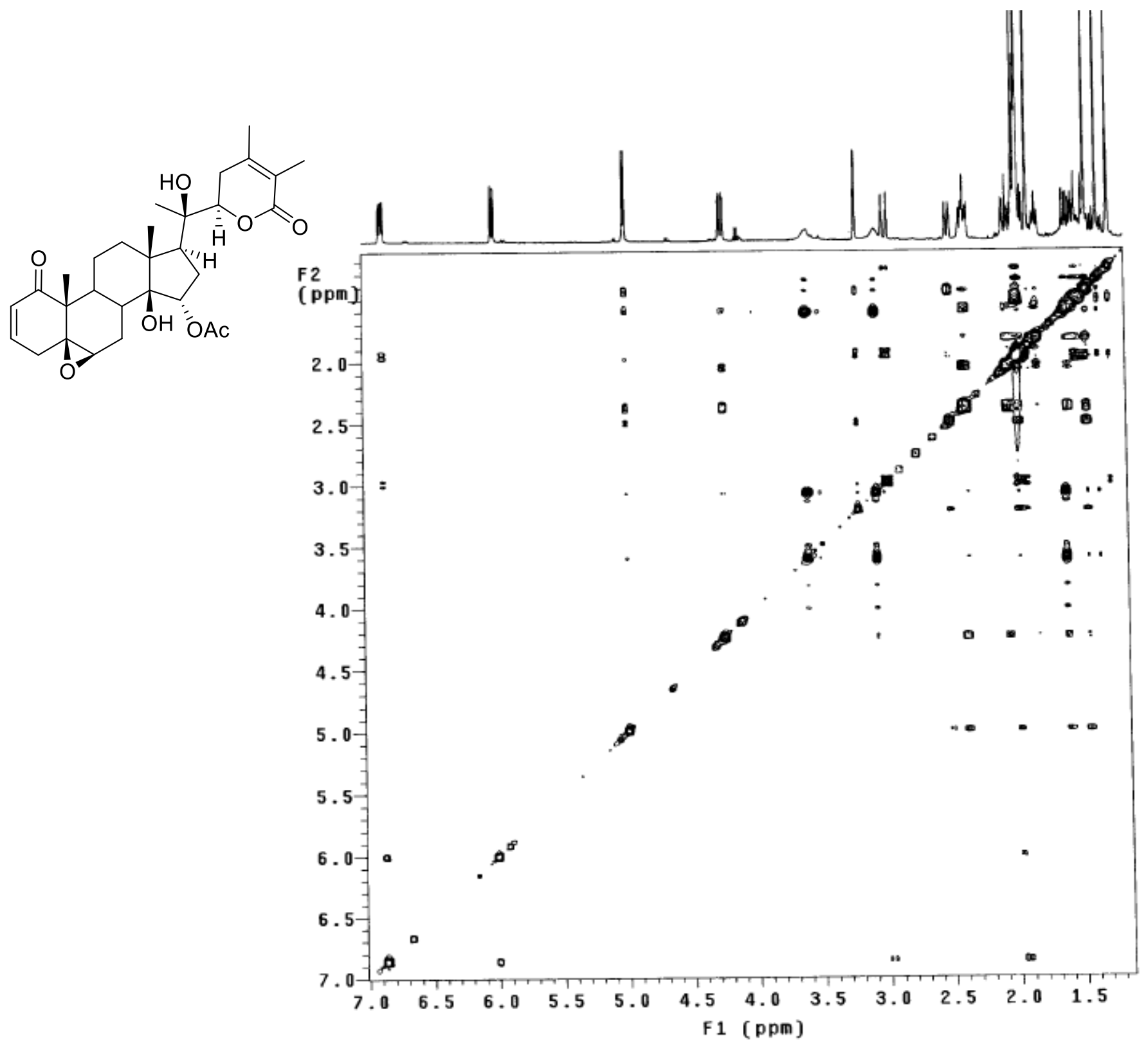

Figure S61. NOESY spectrum of compound $14\left(\mathrm{CDCl}_{3}\right)$. 


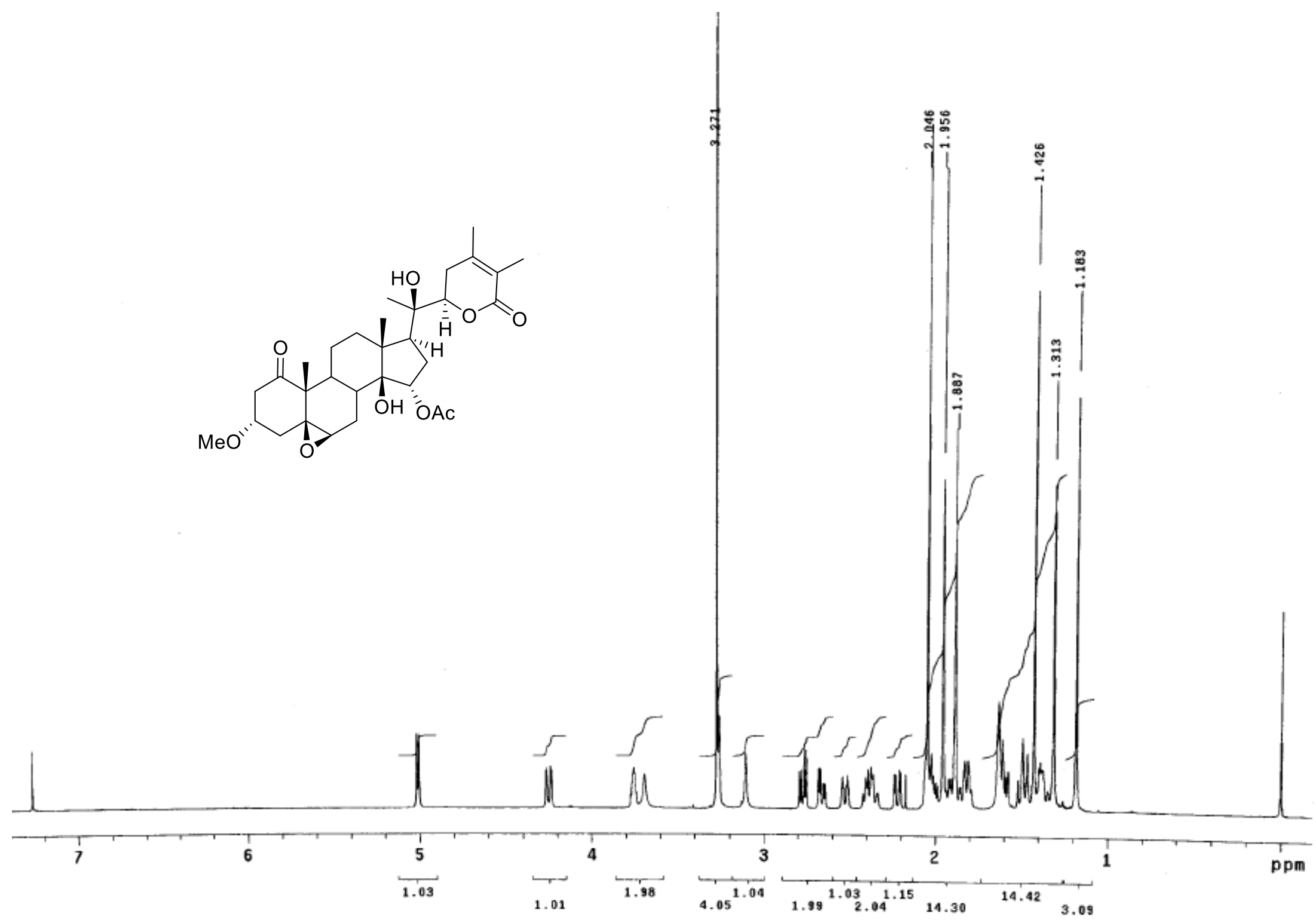

Figure S62. ${ }^{1} \mathrm{H}$ NMR spectrum of compound $15\left(500 \mathrm{MHz}, \mathrm{CDCl}_{3}\right)$. 


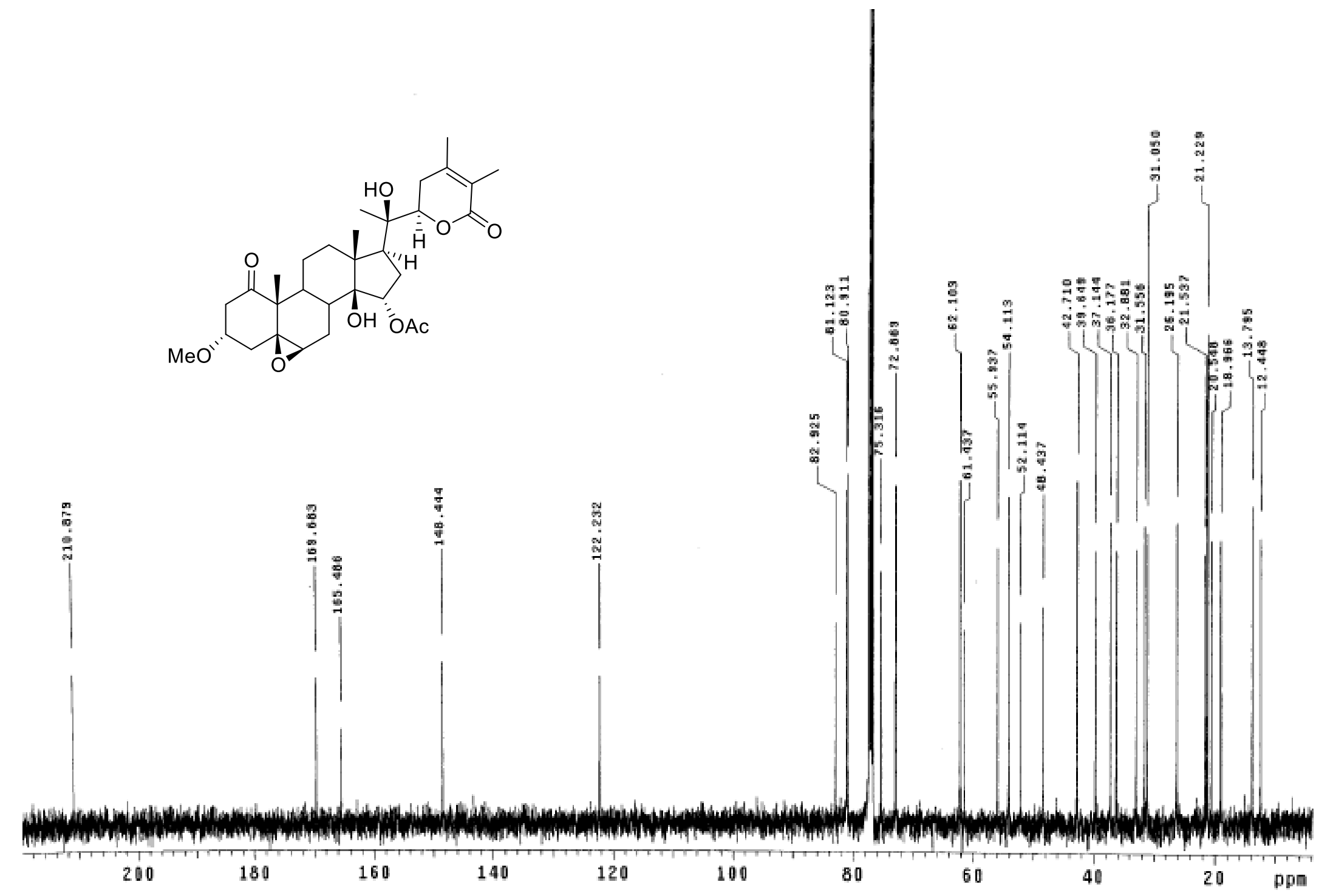

Figure $\mathbf{S 6 3}{ }^{13} \mathrm{C}$ NMR spectrum of compound $15\left(125 \mathrm{MHz}, \mathrm{CDCl}_{3}\right)$. 


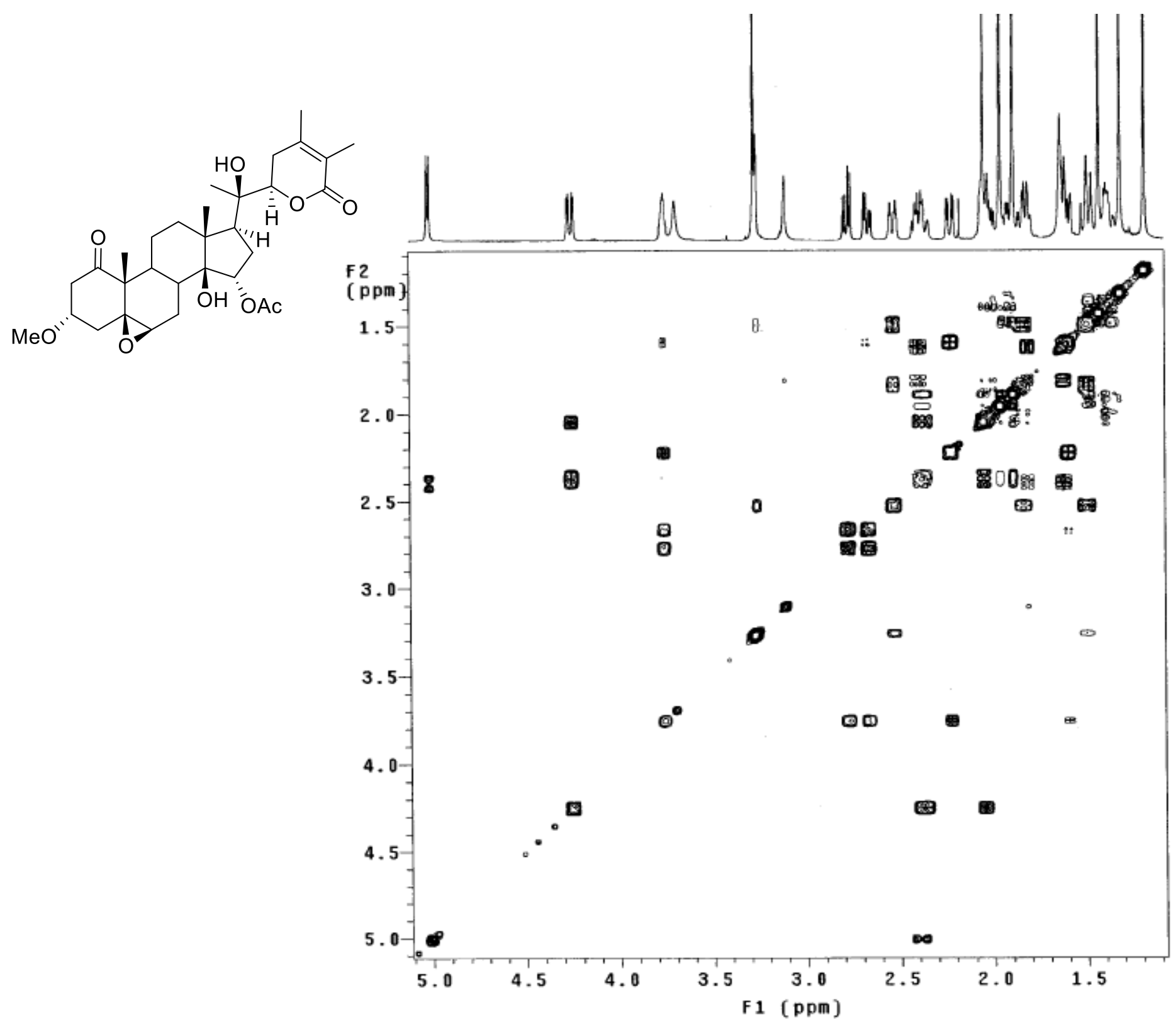

Figure S64. COSY spectrum of compound $15\left(\mathrm{CDCl}_{3}\right)$. 


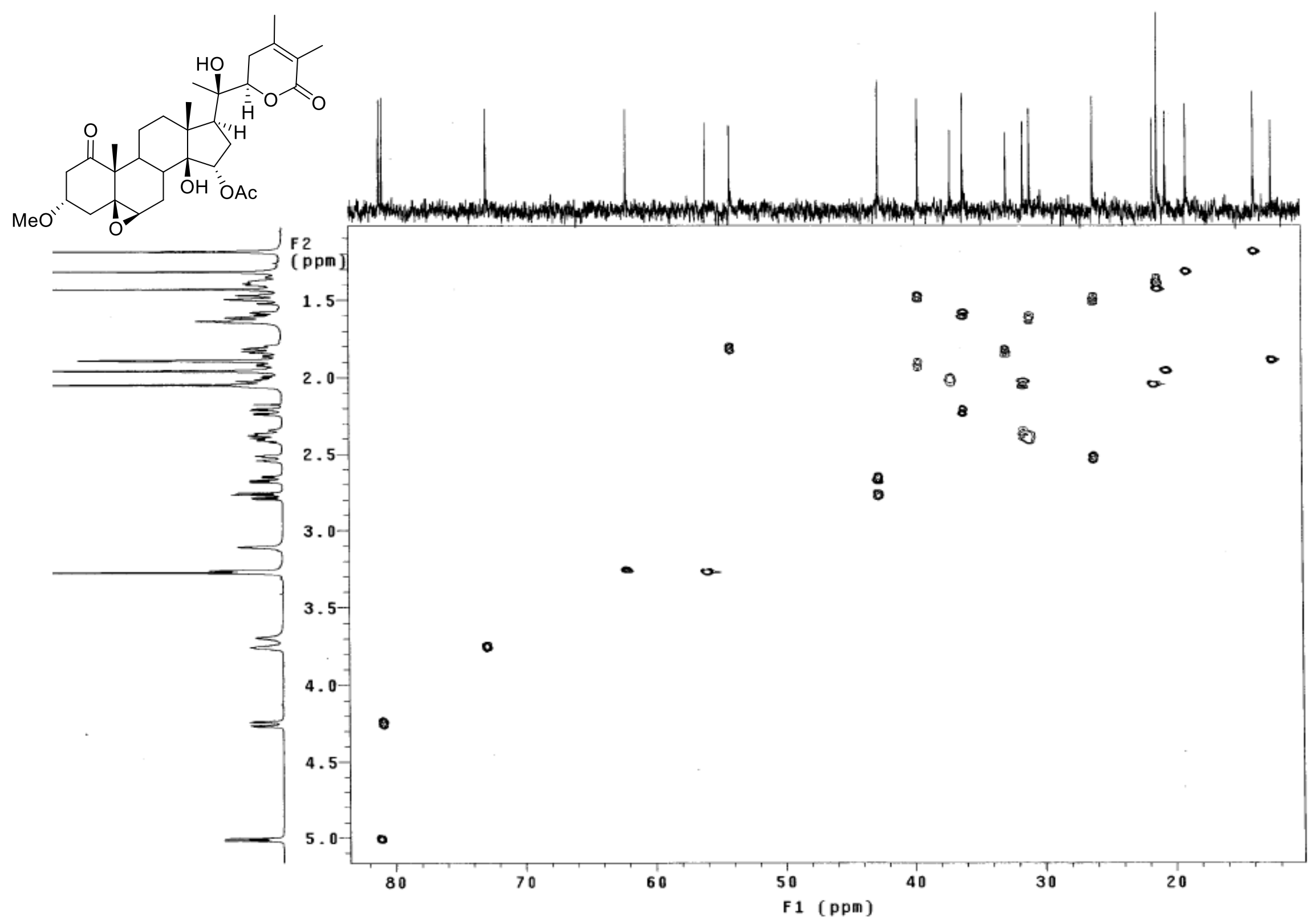

Figure S65. HSQC spectrum of compound $15\left(\mathrm{CDCl}_{3}\right)$. 


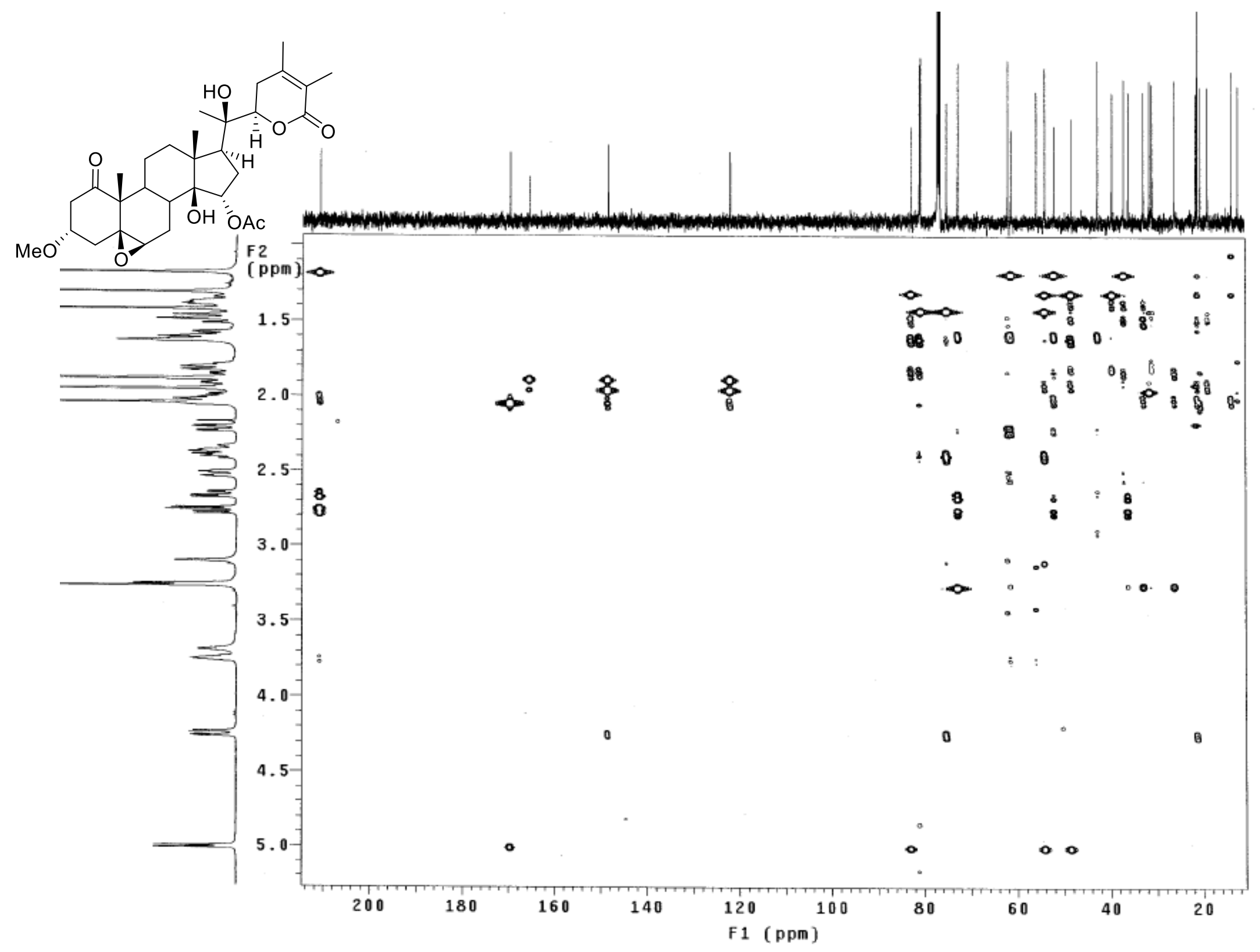

Figure S66. HMBC spectrum of compound $15\left(\mathrm{CDCl}_{3}\right)$. 

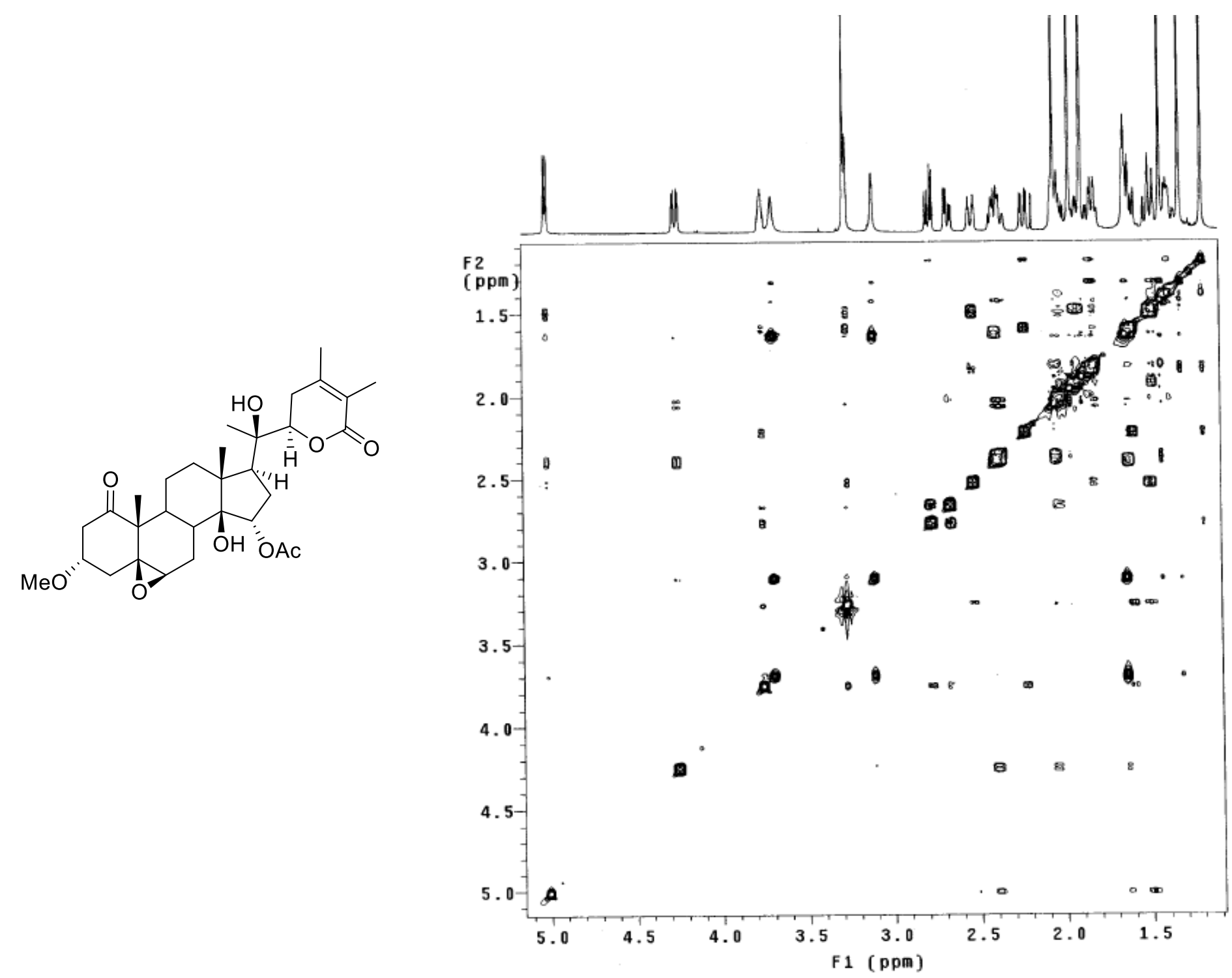

Figure S67. NOESY spectrum of compound $15\left(\mathrm{CDCl}_{3}\right)$. 


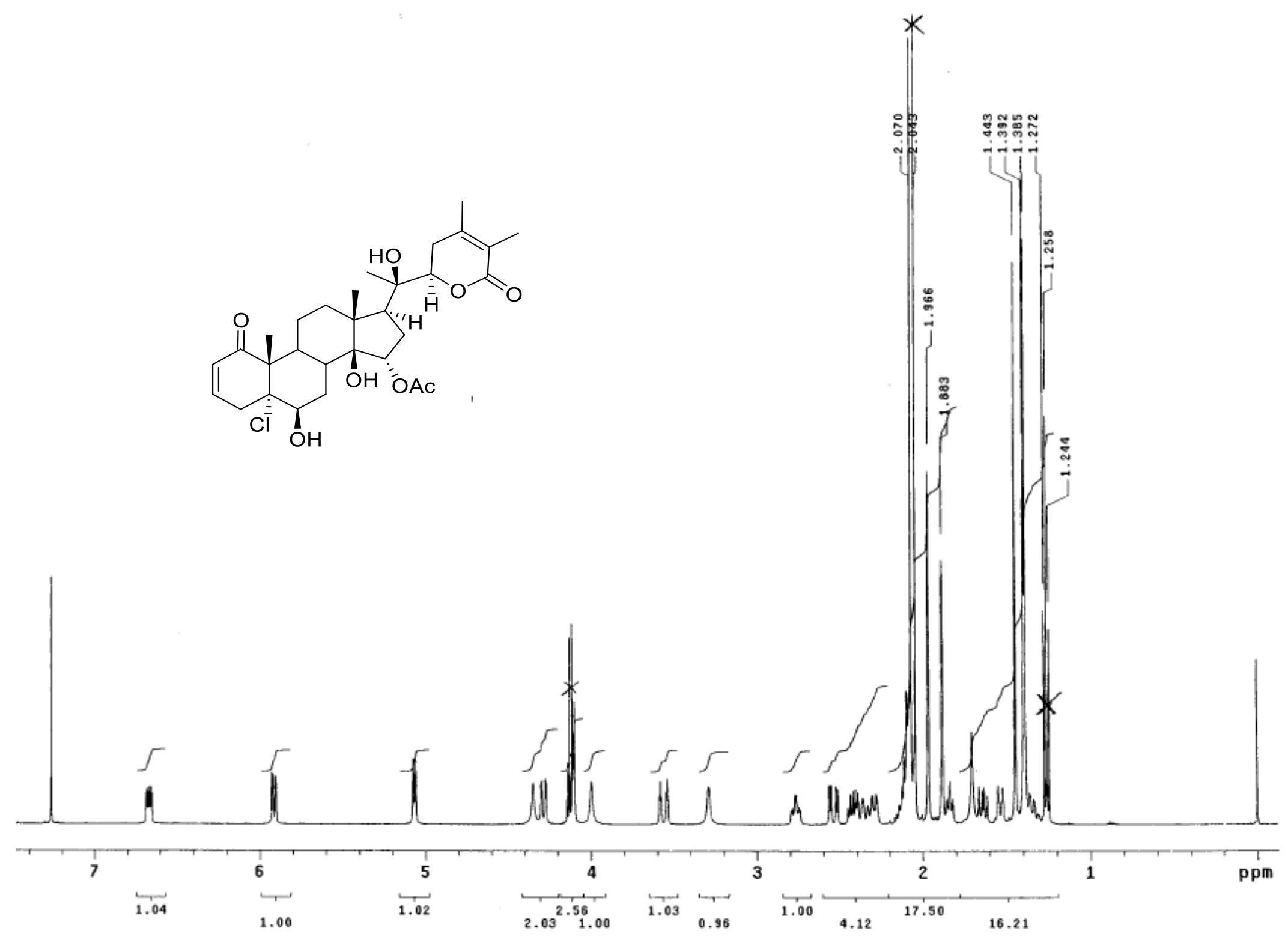

Figure S68. ${ }^{1} \mathrm{H}$ NMR spectrum of compound $16\left(500 \mathrm{MHz}, \mathrm{CDCl}_{3}\right)$, contains EtOAc. 


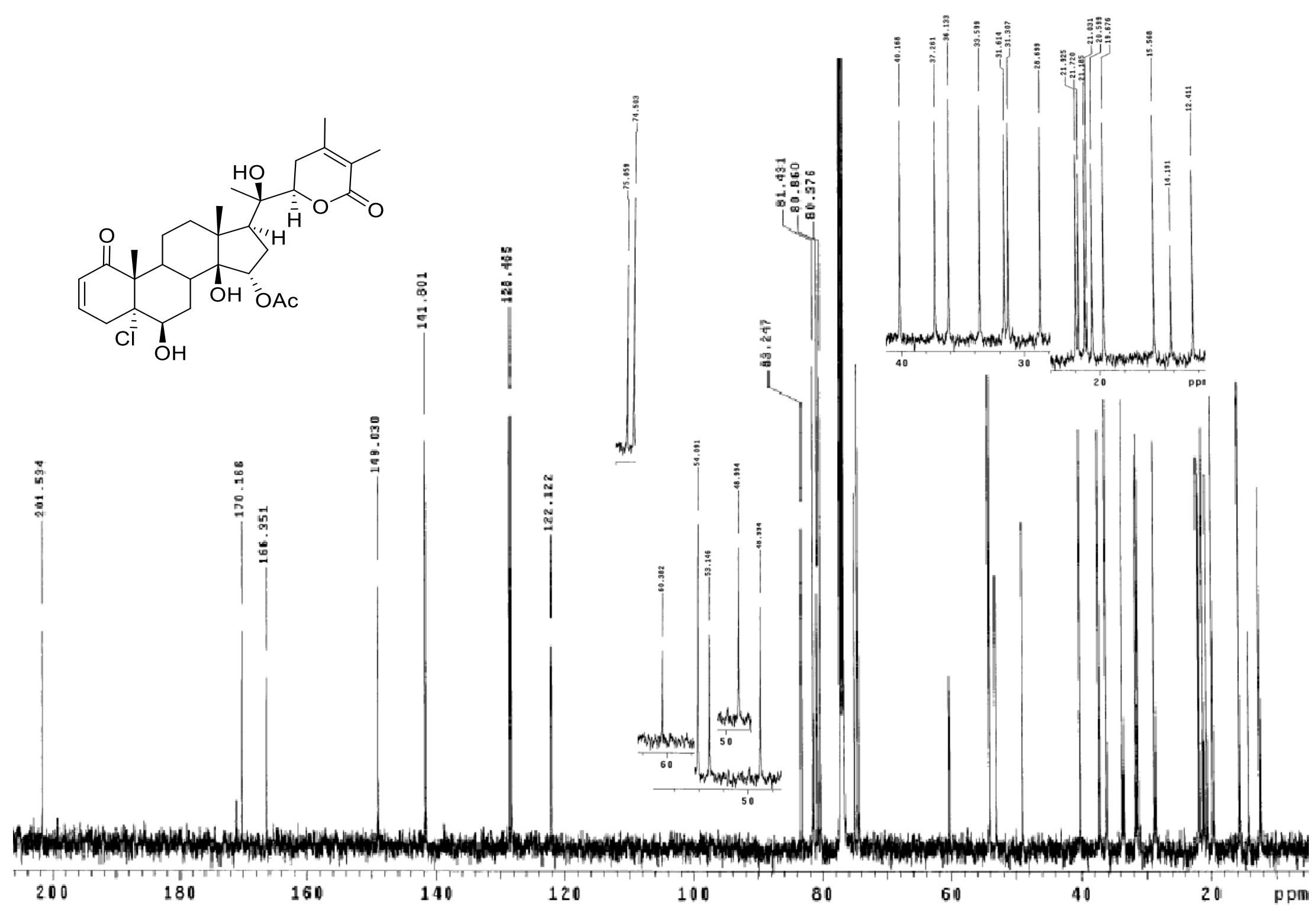

Figure S69. ${ }^{13} \mathrm{C}$ NMR spectrum of compound $16\left(125 \mathrm{MHz}, \mathrm{CDCl}_{3}\right)$, contains EtOAc. 


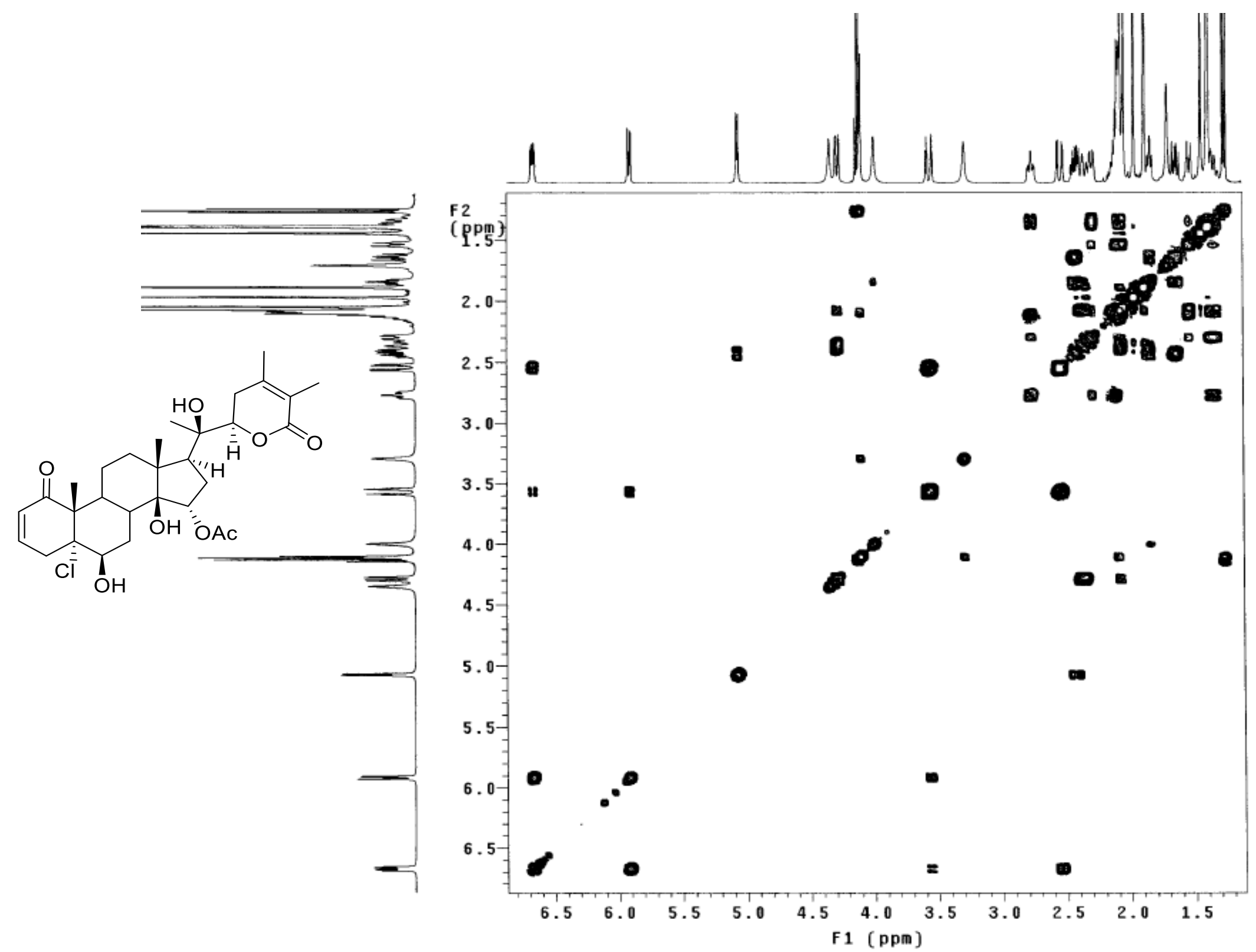

Figure S70. COSY spectrum of compound $\mathbf{1 6}\left(\mathrm{CDCl}_{3}\right)$, contains EtOAc. 


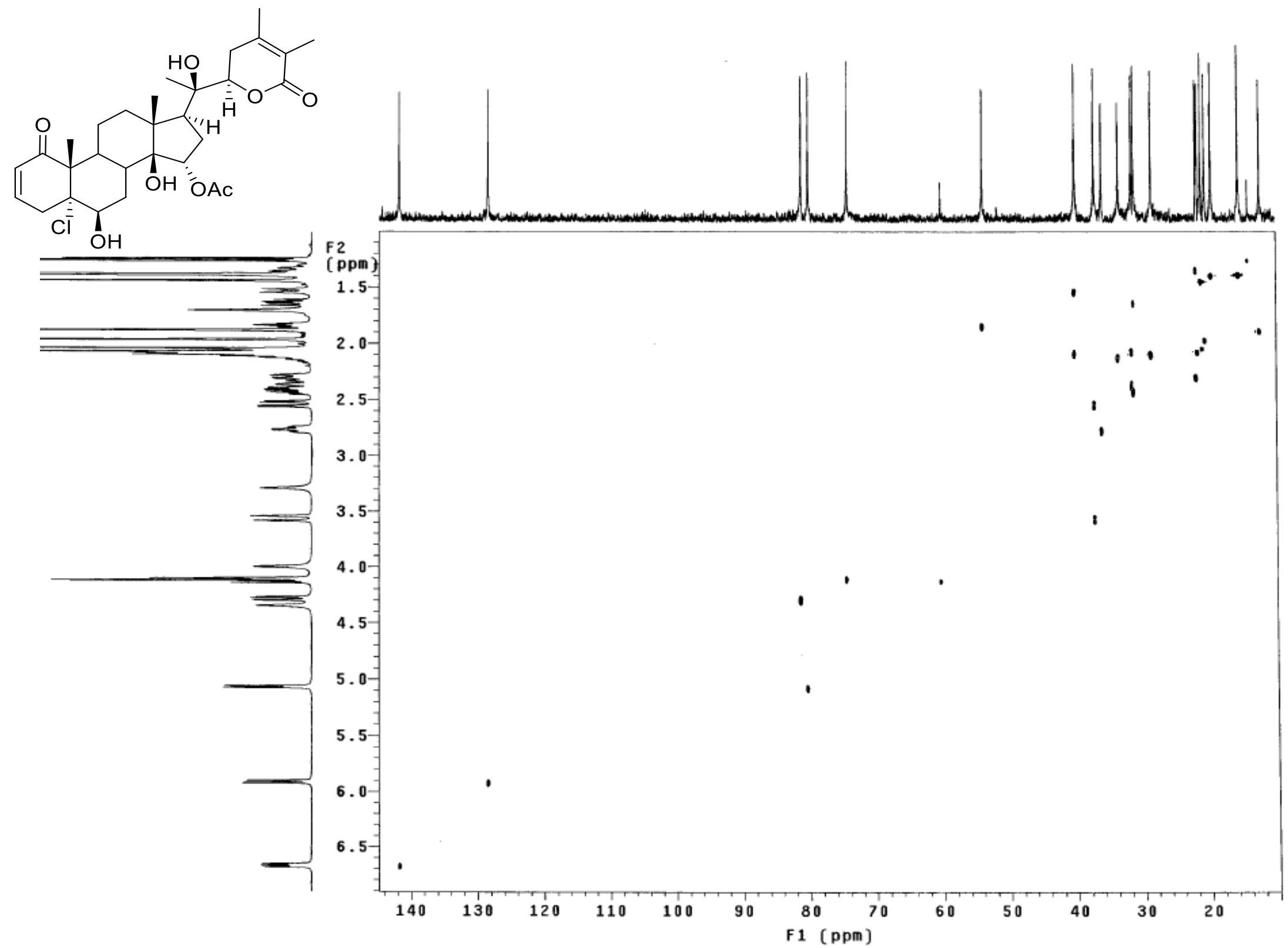

Figure S71. HSQC spectrum of compound $16\left(\mathrm{CDCl}_{3}\right)$, contains EtOAc. 


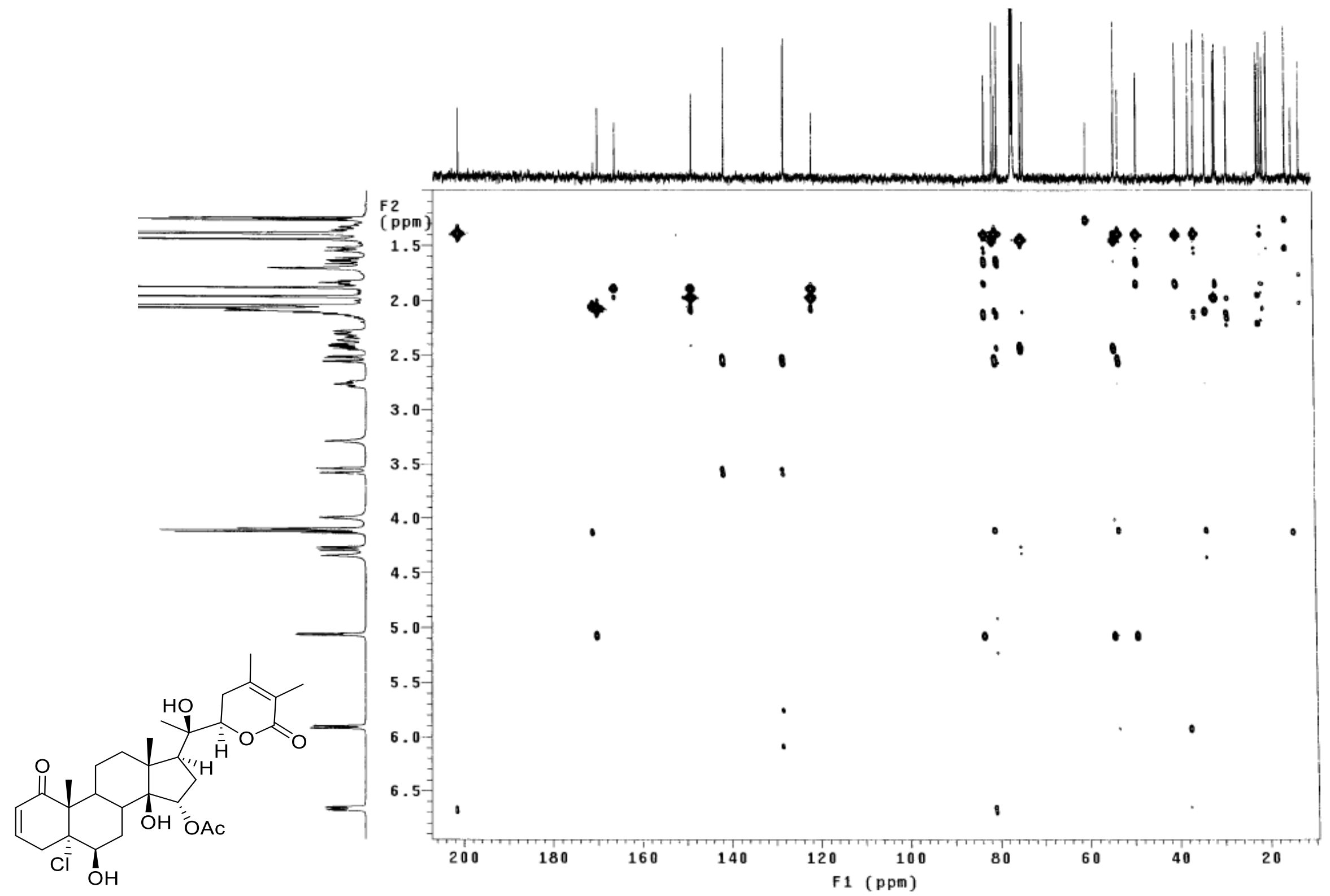

Figure S72. HMBC spectrum of compound $\mathbf{1 6}\left(\mathrm{CDCl}_{3}\right)$, contains EtOAc. 


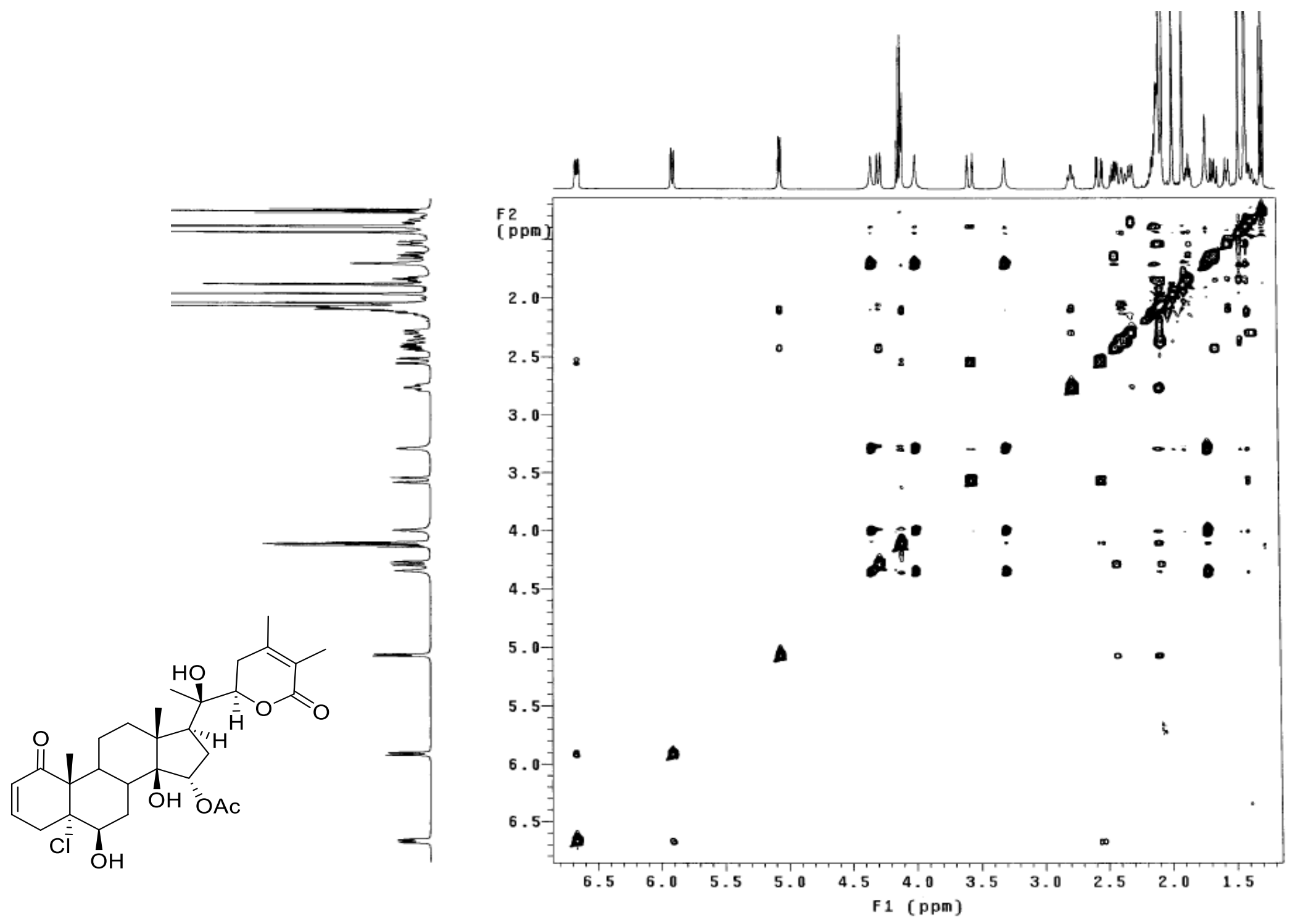

Figure S73. NOESY spectrum of compound $\mathbf{1 6}\left(\mathrm{CDCl}_{3}\right)$, contains EtOAc. 


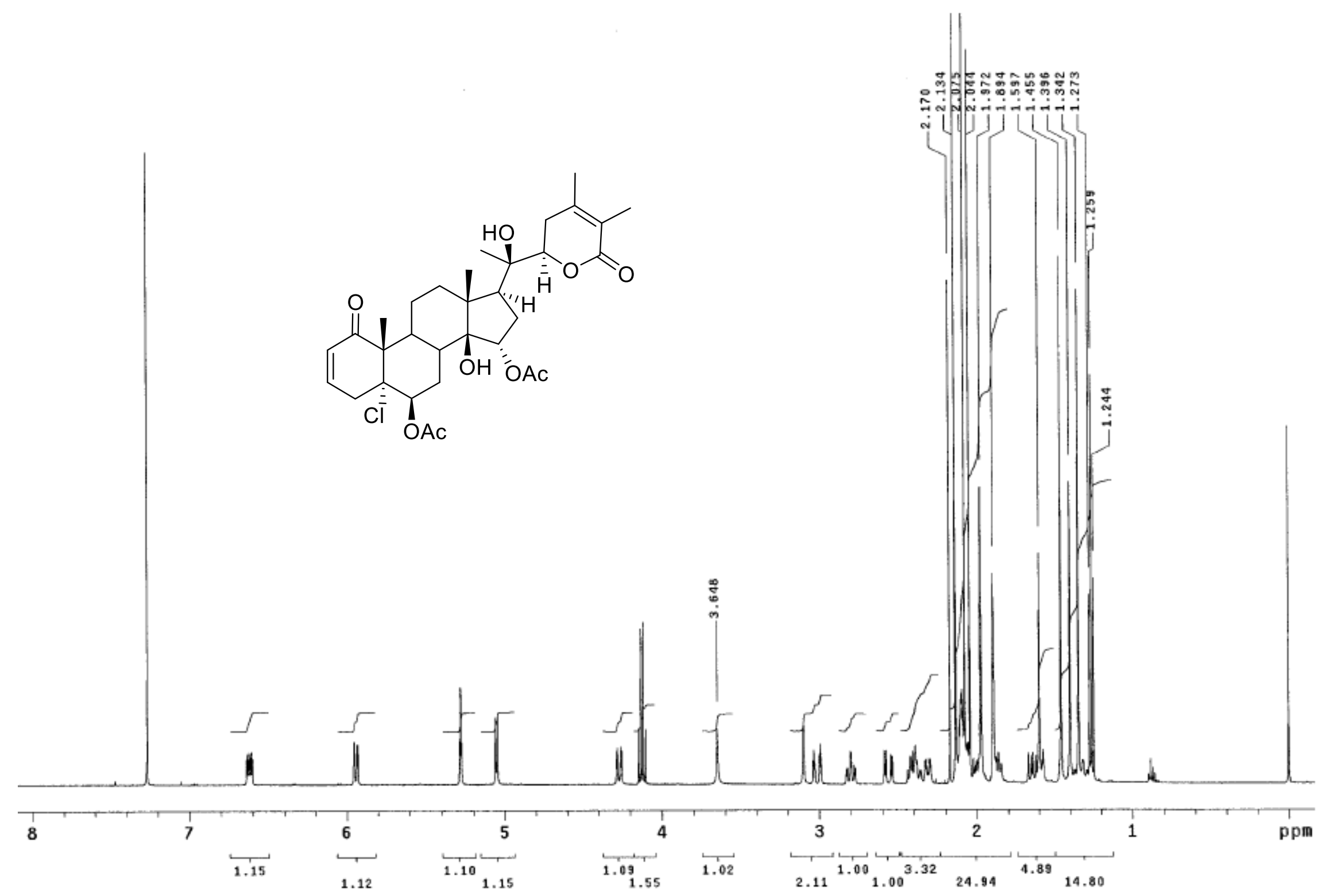

Figure S74. ${ }^{1} \mathrm{H} \mathrm{NMR}$ spectrum of compound $16 \mathbf{a}\left(500 \mathrm{MHz}, \mathrm{CDCl}_{3}\right)$, contains EtOAc. 


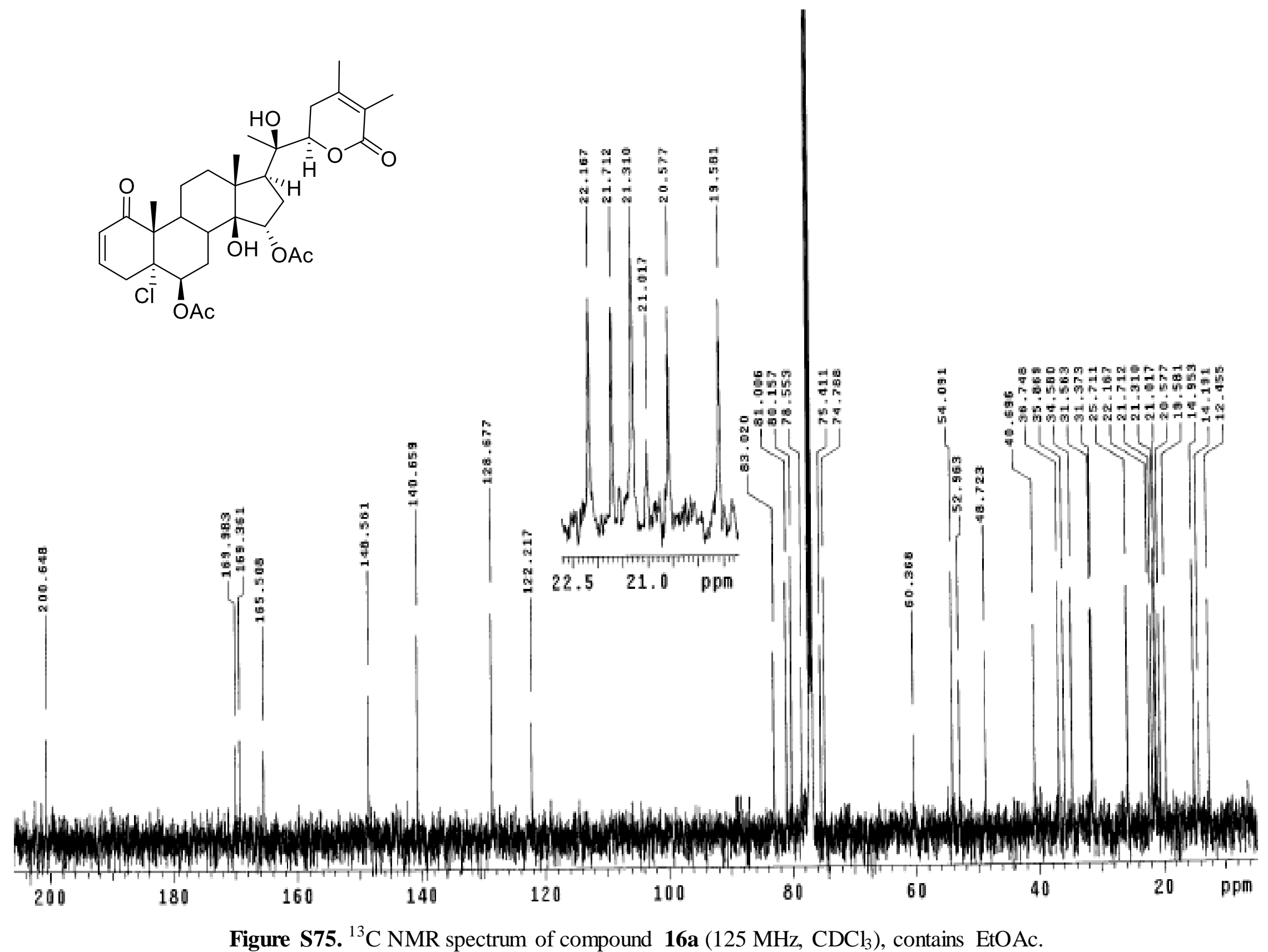




\begin{tabular}{|l|c|}
\hline \multicolumn{2}{|c|}{ Table S3. H-H Distances } \\
\hline $\begin{array}{c}\text { 'Hydrogen } \\
\text { atoms }\end{array}$ & Distance $(\mathbf{A})$ \\
\hline H(60)-H(48) & 2.805 \\
\hline H(61)-H(55) & 2.523 \\
\hline H(60)-H(46) & 2.366 \\
\hline H(48)-H(45) & 2.585 \\
\hline H(47)-H(45) & 2.573 \\
\hline H(46)-H(45) & 2.475 \\
\hline H(49)-H(45) & 2.504 \\
\hline H(62)-H(56) & 2.518 \\
\hline H(61)-H(38) & 2.503 \\
\hline H(47)-H(50) & 2.487 \\
\hline a ChemBio3D-Ultra numbering. \\
\hline
\end{tabular}

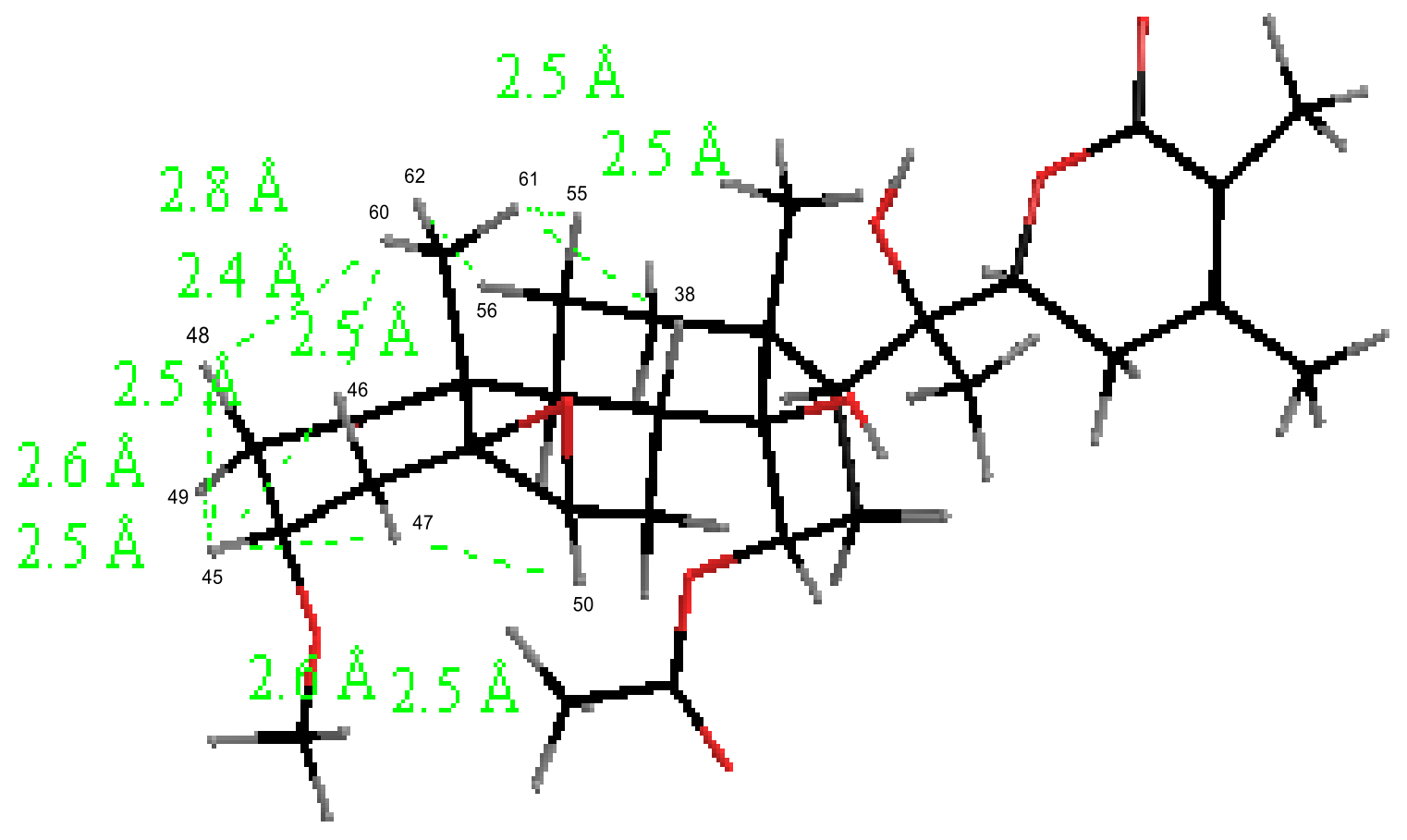

Figure S76 and Table S3. Energy-minimized 3D structure of compound 15 showing selected H-H distances (ChemBio3D-Ultra). 


\section{Acetylcholinestarase inhibition}

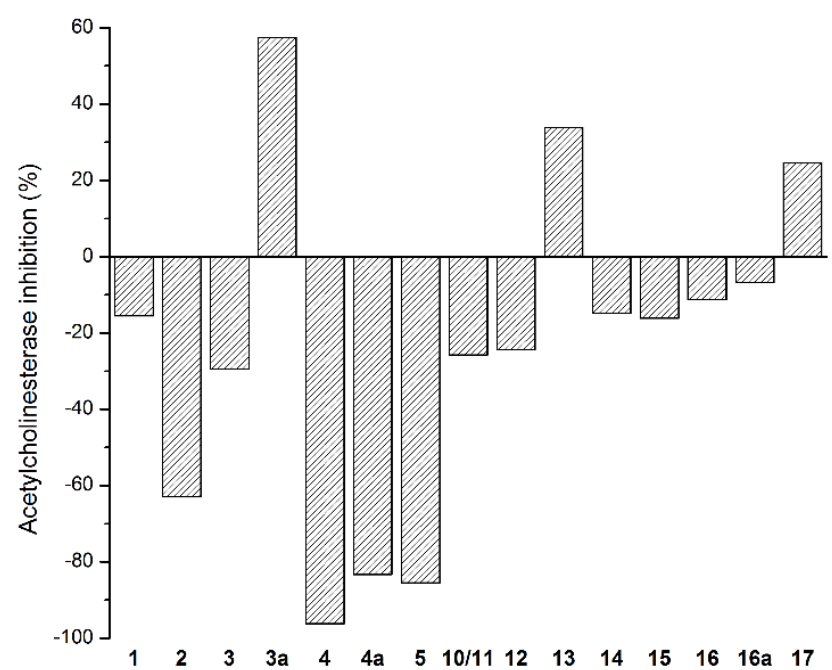

Figure S77. Effect of compounds 1-5, 3a, 4a, 10-17 and 16a (assayed at $100 \mu \mathrm{M}$ ) on the activity of acetylcholinesterase.

Table S4. $\mathrm{IC}_{50}$ values for pumiloxide (3a) and physostigmine for inhibition of $\mathrm{AChE}$.

\begin{tabular}{ccccc}
\hline Compound & $\begin{array}{c}\text { Concentration } \\
(\boldsymbol{\mu} \mathbf{M})\end{array}$ & $\mathbf{A}_{4 \mathbf{2} \mathbf{~ n m}}$ & $\begin{array}{c}\text { Inhibition } \\
(\mathbf{\%})\end{array}$ & $\begin{array}{c}\mathbf{I C}_{\mathbf{5 0}} \\
(\boldsymbol{\mu M})\end{array}$ \\
\hline $\mathbf{3 a}$ & Control & $0.310 \pm 0.024$ & & \\
$(\mathrm{n}=4)$ & 3.16 & $0.260 \pm 0.018$ & $16.66 \pm 7.14$ & $64.43 \pm 9.03$ \\
& 10 & $0.250 \pm 0.016^{*}$ & $19.43 \pm 5.64^{*}$ & \\
& 31.62 & $0.230 \pm 0.008^{*}$ & $24.26 \pm 3.11^{*}$ & \\
& 100 & $0.077 \pm 0.006^{* *}$ & $75.28 \pm 0.46^{* *}$ & \\
\hline Physostigmine & 316.23 & $0.064 \pm 0.015^{* *}$ & $80.08 \pm 3.66^{* *}$ & \\
$(\mathrm{n}=3)$ & Control & $0.320 \pm 0.033$ & & \\
& 0.001 & $0.220 \pm 0.021^{*}$ & $30.57 \pm 1.79^{*}$ & $0.003 \pm 0.0003$ \\
& 0.01 & $0.097 \pm 0.018^{* *}$ & $69.65 \pm 2.45^{* *}$ & \\
\hline
\end{tabular}

Values represent the average of three to four independent experiments \pm standard error of the mean.

The data were subjected to an analysis of variance (ANOVA) followed by a Dunnett's test to isolate groups with significant difference.

Values of $p \leq 0.05(*)$ and $p \leq 0.01(* *)$ indicate significative differences respect to the control 